\title{
Formulas for monodromy
}

\author{
Alan Stapledon*
}

\section{"Correspondence:}

alan.stapledon@sydney.edu.au Department of Mathematics,

University of Sydney, Sydney, NSW 2006, Australia

\begin{abstract}
Given a family $X$ of complex varieties degenerating over a punctured disk, one is interested in computing related invariants called the motivic nearby fiber and the refined limit mixed Hodge numbers, both of which contain information about the induced action of monodromy on the cohomology of a fiber of $X$. Our first main result is that the motivic nearby fiber of $X$ can be computed by first stratifying $X$ into locally closed subvarieties that are nondegenerate in the sense of Tevelev, and then applying an explicit formula on each piece of the stratification that involves tropical geometry. Our second main result is an explicit combinatorial formula for the refined limit mixed Hodge numbers in the case when $X$ is a family of nondegenerate hypersurfaces. As an application, given a complex polynomial, then, under appropriate conditions, we give a combinatorial formula for the Jordan block structure of the action of monodromy on the cohomology of the Milnor fiber, generalizing a famous formula of Varchenko for the associated eigenvalues. In addition, we give a formula for the Jordan block structure of the action of monodromy at infinity.
\end{abstract}

Keywords: Tropical geometry, Monodromy, Motivic nearby fiber, Hodge theory, Polytopes, Ehrhart theory, Monodromy at infinity, Milnor fiber

\section{Contents}

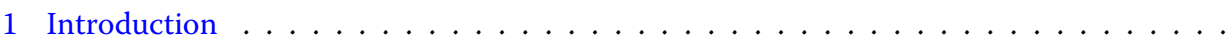

1.1 Organization of the paper $\ldots \ldots \ldots \ldots \ldots \ldots$

2 Geometry of degenerations

2.1 The equivariant Grothendieck ring . . . . . . . . . . . . . .

2.2 The motivic nearby fiber . . . . . . . . . . . . . . . . . . .

2.3 Motivic invariants . . . . . . . . . . . . . . . .

3 Tropical geometry

4 The combinatorics of height functions . . . . . . . . . . . . .

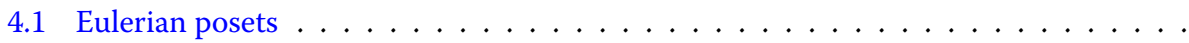

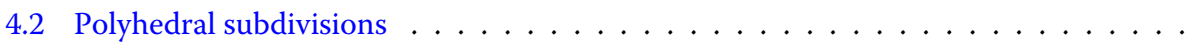

4.3 Weighted Ehrhart theory $\ldots \ldots \ldots \ldots \ldots \ldots$

4.4 Mixed invariants . . . . . . . . . . . . . . . . .

5 Degenerations of hypersurfaces .

6 Applications to the monodromy of complex polynomials . . . . . . . . . . .

6.1 Degenerations of hypersurfaces in affine space $\ldots \ldots \ldots \ldots$

6.2 Jordan block structure of monodromy at infinity . . . . . . . . . . . . .

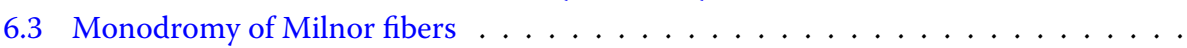

References

(c) The Author(s) 2017. This article is distributed under the terms of the Creative Commons Attribution 4.0 International License (http://creativecommons.org/licenses/by/4.0/), which permits unrestricted use, distribution, and reproduction in any medium, provided you give appropriate credit to the original author(s) and the source, provide a link to the Creative Commons license, and indicate if changes were made. 


\section{Introduction}

Before introducing the main results of this paper, we first present an application. Let $f\left(x_{1}, \ldots, x_{n}\right) \in \mathbb{C}\left[x_{1}, \ldots, x_{n}\right]$ be a complex polynomial with no constant term, and consider the induced map $f: \mathbb{C}^{n} \rightarrow \mathbb{C}$. One would like to understand the singularity of $f^{-1}(0) \subseteq \mathbb{C}^{n}$ at the origin (we assume this is an isolated singularity). A classical invariant used to distinguish the singularities arising from different polynomials is the action of monodromy on the cohomology of the associated Milnor fiber. In simple terms, this means associating to $f$ a finite, square complex matrix $M_{f}$ that is well defined up to change of basis. We assume that $f$ is 'general' in the sense that it is nondegenerate with respect to its Newton polyhedron $\Gamma_{+}(f)$, and also that it is convenient (see Sect. 6.3). The most famous result was proved by Varchenko in 1976 [61], who gave an explicit combinatorial formula for the eigenvalues (with multiplicity) of $M_{f}$, involving alternating signs of volumes of polytopes associated with $\Gamma_{+}(f)$. Unfortunately, since $M_{f}$ is rarely diagonalizable, it is not, in general, determined by its eigenvalues. We are left with the problem of giving combinatorial formulas for the numbers $J_{k, \alpha}$ of Jordan blocks of $M_{f}$ of size $k$ with eigenvalue $\alpha$. Using tropical geometry, together with weighted Ehrhart theory, which involves the enumerative combinatorics of lattice points in dilates of polytopes, we settle this question:

Theorem 1.1 (Corollary 6.22) Let $f \in \mathbb{C}\left[x_{1}, \ldots, x_{n}\right]$ be a complex polynomial such that $f^{-1}(0)$ admits an isolated singularity at the origin. Assume further that $f$ is convenient and nondegenerate with respect to its Newton polyhedron $\Gamma_{+}(f)$. Then there are explicit, nonnegative combinatorial formulas for the numbers $J_{k, \alpha}$, involving the weighted Ehrhart theory of polytopes associated with $\Gamma_{+}(f)$.

We refer the reader to Corollary 6.22 for the specific formulas. By a 'nonnegative' formula, we mean that each $J_{k, \alpha}$ is expressed as a sum of nonnegative integers. By summing over $k$, we obtain a nonnegative formula for the multiplicities of the eigenvalues of $M_{f}$, which is equivalent to Varchenko's formula (Remark 6.19). Using different techniques, special cases of formulas for the $J_{k, \alpha}$ have previously been proved by Matsui and Takeuchi [40] and van Doorn and Steenbrink [60], and an algorithm to compute the numbers $J_{k, \alpha}$ involving simplicial resolutions of toric varieties was proved by Matsui and Takeuchi in [40], extending work of Danilov [13] and Tanabé [58]. We note that algorithms in a more general setting are given by Schulze in [49].

The theorem above is a corollary of more general results that we will outline in the remainder of the introduction. Given a family of varieties $f: X \rightarrow \mathbb{D}^{*}$ over a complex disk, Denef and Loeser [17] associate an invariant $\psi_{X}$ called the motivic nearby fiber, which contains information about the variation of Hodge structures associated with the degeneration. In particular, if we fix a nonzero fiber $X_{\text {gen }}:=f^{-1}(t)$ for some sufficiently small $t \in \mathbb{D}^{*}$, then $\psi_{X}$ encodes information on the induced monodromy map $T: H_{c}^{m}\left(X_{\text {gen }}\right) \rightarrow H_{c}^{m}\left(X_{\text {gen }}\right)$, which is a linear operator on the complex cohomology with compact supports of $X_{\text {gen }}$ and is quasi-unipotent, i.e., $T=T_{s} T_{u}$, where $T_{s}$ and $T_{u}$ commute, $T_{S}$ is semi-simple and has finite order, and $T_{u}$ is unipotent. In [30], Eric Katz and the author showed that one can use tropical geometry to concretely compute a specialization of $\psi_{X}$ that is invariant under base change, and hence encodes information about $T_{u}$ but not $T_{s}$. Using new combinatorics developed in [29], in the case of families of schön complex hypersurfaces of tori, explicit combinatorial formulas were then deduced for the refined 
limit mixed Hodge numbers, which, in particular, determine the Jordan block structure of the action of $T_{u}$ on the graded pieces (with respect to the Deligne weight filtration) of $H_{c}^{m}\left(X_{\text {gen }}\right)$. Here schön is a 'generic' condition introduced by Tevelev [59], generalizing the notion of nondegeneracy of a hypersurface of a complex torus [33]. Equivalent formulas were also given for the action of $T_{u}$ on the intersection cohomology groups of a family of schön hypersurfaces of a projective toric variety.

The goal of this note is to extend the above results to the full generality of the motivic nearby fiber $\psi_{X}$ and monodromy operator $T$. Our first main result is to show that the motivic nearby fiber may be computed using tropical geometry (see Sect. 3). A key point is that since there is a range of available software implementing the main algorithms in tropical geometry [26,27], our main formula Theorem 3.2 can be computed in practice.

Weighted Ehrhart theory was introduced by the author in [52] both in order to extend and reprove many results in Ehrhart theory, the study of enumeration of lattice points in polytopes, and in order to give explicit computations of motivic integrals on toric stacks [53]. Roughly speaking, one associates with every lattice point $v$ a 'weight' $w(v)$ in $\mathbb{Q} / \mathbb{Z}$ and then attempts to enumerate lattice points in polytopes keeping track of the associated weights. Extending work in [29], in Sect. 4.4, we associate new Ehrhart-theoretic invariants to a pair $(P, v)$, where $P$ is a lattice polytope and $v$ is the convex graph of an integral height function on $P$. In particular, we introduce the weighted refined limit mixed $h^{*}$-polynomial $h^{*}(P, v ; u, v, w) \in \mathbb{Z}[\mathbb{Q} / \mathbb{Z}][u, v, w]$. Roughly speaking, our second main result states that if $X^{\circ}$ is a family of schön complex hypersurfaces of tori, then the associated equivariant refined limit mixed Hodge numbers are precisely the coefficients of $h^{*}(P, v ; u, v, w)$ (Theorem 5.7, Corollary 5.12), where $(P, v)$ is the Newton polytope and associated convex graph associated with $X^{\circ}$. In particular, this gives combinatorial formulas for the Jordan block structure of the action of $T$ on the graded pieces (with respect to the Deligne weight filtration) of $H_{c}^{m}\left(X_{\text {gen }}^{\circ}\right)$. Equivalent formulas are also given for the equivariant refined limit mixed Hodge numbers associated with the intersection cohomology groups of a family of schön hypersurfaces of a projective toric variety (Theorem 5.9, Corollary 5.12). In Example 5.13, we express these invariants in a special case in terms of dimensions of orbifold cohomology $[11,12]$ of a toric stack [4]. It would be interesting to have an explanation of this fact involving 'mirror symmetry'.

As a special case of the above result, in Theorem 5.11, we give a formula for the equivariant Hodge-Deligne polynomial of a schön complex hypersurface of a torus invariant under the action of an element of the torus of finite order. An algorithm to determine this polynomial had previously been given by Matsui and Takeuchi in [39], generalizing an algorithm of Danilov and Khovanskii in the nonequivariant setting [14]. Moreover, the above formula reduces to a formula of Borisov and Mavlyutov [5] in the nonequivariant setting, which itself is a simplification of a formula of Batyrev and Borisov [3].

We next present some applications to the monodromy of complex polynomials. Let $f\left(x_{1}, \ldots, x_{n}\right) \in \mathbb{C}\left[x_{1}, \ldots, x_{n}\right]$ be a complex polynomial. It is well known that there exists a minimal finite subset $B_{f} \subseteq \mathbb{C}$, such that $f: \mathbb{C}^{n} \rightarrow \mathbb{C}$ is a locally trivial fibration away from $B_{f}$. Then monodromy defines an action of the fundamental group $\pi_{1}\left(\mathbb{C} \backslash B_{f}\right)$ on the cohomology of a fixed generic fiber of $f$. On the one hand, we are interested in the monodromy action induced by moving anti-clockwise around a small loop around an element of $b \in B_{f}$. After translating $f$ by a constant, we may assume that $b=0$, and then the resulting action is called monodromy at $\mathbf{0}$. On the other hand, choose $R>0$ sufficiently large 
such that $B_{f}$ is strictly contained in $\{z \in \mathbb{C}|| z \mid=R\}$. Then the monodromy action on cohomology induced by moving clockwise around such a loop is called the monodromy at infinity of $f$, and a fundamental result of Dimca and Némethi [18] states that monodromy at infinity essentially determines the monodromy action of $\pi_{1}\left(\mathbb{C} \backslash B_{f}\right)$. We assume that $f$ is convenient in the sense that its Newton polytope has nonzero intersection with each ray through a coordinate vector [34]. Then under certain schönness (also called 'nondegeneracy') conditions, we give explicit combinatorial formulas that essentially completely describe both monodromy at 0 (Example 6.7) and monodromy at infinity (Example 6.8). In particular, we deduce 'nonnegative' combinatorial formulas for both the equivariant limit mixed Hodge numbers associated with monodromy at infinity (Corollary 6.13) and the Jordan block structure of monodromy at infinity (Corollary 6.14). Algorithms to compute the latter invariants were given by Matsui and Takeuchi in [39]. Also, these formulas specialize to known formulas for the spectrum at infinity of $f$ [39, Theorem 5.11] and zeta function at infinity of $f$ [35]. We note that some of the above results may be extended without the assumption that $f$ is convenient (see Remark 6.1). We also present analogous 'local' results for the Milnor fiber of a polynomial $f$ with an isolated singularity at the origin, including combinatorial formulas for both the corresponding equivariant limit mixed Hodge numbers, and, as outlined at the beginning of this introduction, the Jordan block structure of the action of monodromy on the cohomology of the Milnor fiber.

Finally, we mention some possible generalizations of the above results that we expect will follow by similar methods. Firstly, using the 'Cayley trick' of Danilov and Khovanskii [14, Section 6], one may extend our results on hypersurfaces to the case of complete intersections. In particular, in the case of the monodromy at infinity and monodromy of the cohomology of the Milnor fiber, one may obtain explicit formulas that extend algorithms given by Esterov and Takeuchi in [23]. Secondly, in the case when $f$ is not convenient, one may use the results above to obtain formulas extending algorithms of Takeuchi and Tibar in [57]. Lastly, analogous formulas to those in Sect. 6.3 for families of hypersurfaces of an affine toric variety (rather than simply affine space) may be obtained using the setup and results of Steenbrink in [56].

\subsection{Organization of the paper}

This paper is structured as follows. In Sect. 2, we review the necessary geometric background. In Sect. 3, we show how to compute the motivic nearby fiber using tropical geometry. In Sect. 4, we review the necessary combinatorial background before introducing our main combinatorial invariants. In Sect. 5, we prove our combinatorial formulas for geometric invariants associated with degenerations of hypersurfaces. Finally, in Sect. 6, we apply our results to deduce formulas for invariants associated with the monodromy of complex polynomials.

Notation and conventions All cohomology has complex coefficients. If $N$ is a lattice, then $N_{\mathbb{R}}=N \otimes_{\mathbb{R}} \mathbb{R}$. We identify the group $\mathbb{Q} / \mathbb{Z}$ with the group $\mathbb{S}_{\mathbb{Q}}^{1}$ of rational points on the circle $\{z \in \mathbb{C}|| z \mid=1\}$, sending $[k] \in \mathbb{Q} / \mathbb{Z}$ to $\alpha=e^{2 \pi \sqrt{-1} k} \in \mathbb{S}_{\mathbb{Q}}^{1}$. We fix $\mathbb{K}=\mathbb{C}(t)$. A variety $X$ over $\mathbb{K}$ is naturally interpreted as a complex variety with a morphism $f: X \rightarrow \mathbb{D}^{*}$, for some sufficiently small punctured complex disk $\mathbb{D}^{*}$ around the origin, and $X_{\text {gen }}:=f^{-1}(t)$ denotes a fixed fiber for some choice of $t \in \mathbb{D}^{*}$. 


\section{Geometry of degenerations}

In this section we briefly recall the notion of the motivic nearby fiber and its associated geometry. We refer the reader to Bittner [7], Peters [43] and Peters and Steenbrink [44] for details. A similar exposition is given in the nonequivariant setting in [30, Section 3].

\subsection{The equivariant Grothendieck ring}

We follow the treatment in [7] below. Fix a field $k$. The Grothendieck ring $K_{0}\left(\operatorname{Var}_{k}\right)$ of algebraic varieties over $k$ is the free abelian group generated by isomorphism classes $[V]$ of varieties $V$ over $k$, modulo the relation

$$
[V]=[U]+[V \backslash U]
$$

whenever $U$ is an open subvariety of $V$. Multiplication is given by tensor product of varieties.

Suppose that $G$ is a finite group. An (algebraic) action of $G$ on a variety over $k$ is good if every orbit lies in an open affine subvariety. Then we may similarly define $\widetilde{K}_{0}^{G}\left(\operatorname{Var}_{k}\right)$ to be the free abelian group generated by isomorphism classes $[V]$ of varieties $V$ over $k$ together with a good action of $G$, modulo the relation $[V]=[U]+[V \backslash U]$, whenever $U$ is a $G$-invariant open subvariety of $V$. The equivariant Grothendieck ring $K_{0}^{G}\left(\operatorname{Var}_{k}\right)$ is the quotient of $\widetilde{K}_{0}^{G}\left(\operatorname{Var}_{k}\right)$ by the relation $[\mathbb{P}(V) \circlearrowleft G]=\left[\mathbb{P}^{n} \times(X \circlearrowleft G)\right]$, where $V \rightarrow X$ is a vector bundle of rank $n+1$ with a good $G$-action that is linear over a good $G$-action on $X$. Here $\mathbb{P}(V)$ denotes the projectivization of $V$ with induced $G$-action, and $\mathbb{P}^{n}$ admits a trivial $G$-action. A group homomorphism $G \rightarrow H$ induces a ring homomorphism $K_{0}^{H}\left(\operatorname{Var}_{k}\right) \rightarrow K_{0}^{G}\left(\operatorname{Var}_{k}\right)$ by restriction. In particular, $K_{0}^{G}\left(\operatorname{Var}_{k}\right)$ is a module over $K_{0}\left(\operatorname{Var}_{k}\right)$, and forgetting the action of $G$ gives a ring homomorphism $K_{0}^{G}\left(\operatorname{Var}_{k}\right) \rightarrow K_{0}\left(\operatorname{Var}_{k}\right)$. We will follow the convention that $\mathbb{L}:=\left[\mathbb{A}^{1}\right]$ is the class of the affine line with trivial $G$-action. A motivic invariant is a ring homomorphism $K_{0}^{G}\left(\operatorname{Var}_{k}\right) \rightarrow R$, for some $\operatorname{ring} R$.

Remark 2.1 Suppose that a finite group $G$ acts linearly on a $k$-vector space $V$ of dimension $n+1$, i.e., $V$ is a representation of $G$. Then taking $X=p t$ in the condition above, we see that $[\mathbb{P}(V)]=\left[\mathbb{P}^{n}\right]=\mathbb{L}^{n}+\mathbb{L}^{n-1}+\cdots+1 \in K_{0}^{G}\left(\operatorname{Var}_{k}\right)$.

Now assume that $k=\mathbb{C}$ and identify $\mu_{n} \cong \mathbb{Z} / n \mathbb{Z}$ with the group of $n^{\text {th }}$ roots of unity in $\mathbb{C}$ generated by $\exp (2 \pi \sqrt{-1} i / n)$. The epimorphisms $\mu_{n d} \rightarrow \mu_{n}, \zeta \mapsto \zeta^{d}$ give rise to a projective limit $\widehat{\mu}=\lim \mu_{n}$. If $g$ is an element of a group $G$ of finite order, then we have a well-defined group homomorphism $\widehat{\mu} \rightarrow \mu_{n} \rightarrow G$ sending $\exp (2 \pi \sqrt{-1} i / n) \mapsto g$ whenever $g^{n}=1$. In this way, group actions of elements of finite order give rise to group actions of $\widehat{\mu}$.

A good $\widehat{\mu}$-action on a complex variety is an action of $\widehat{\mu}$ induced by a good $\mu_{n}$-action for some $n>0$. The equivariant Grothendieck ring $K_{0}^{\widehat{\mu}}\left(\operatorname{Var}_{\mathbb{C}}\right)$ is defined similarly as above, and coincides with the direct limit of the restriction maps $K_{0}^{\mu_{n}}\left(\operatorname{Var}_{\mathbb{C}}\right) \rightarrow K_{0}^{\mu_{\text {nd }}}\left(\operatorname{Var}_{\mathbb{C}}\right)$ induced by the epimorphisms $\mu_{n d} \rightarrow \mu_{n}, \zeta \mapsto \zeta^{d}$.

Example 2.2 Let $v=\left(v_{1}, \ldots, v_{n}\right) \in \mathbb{Q}^{n}$. Then multiplication by $\left(e^{2 \pi \sqrt{-1} v_{1}}, \ldots, e^{2 \pi \sqrt{-1} v_{n}}\right)$ gives a good $\widehat{\mu}$-action on $\left(\mathbb{C}^{*}\right)^{n}$. This action extends to $\mathbb{P}^{n}$, which admits a natural stratification into tori each invariant under $\widehat{\mu}$, and it follows from Remark 2.1 and induction on dimension that $\left[\left(\mathbb{C}^{*}\right)^{n} \circlearrowleft \widehat{\mu}\right]=(\mathbb{L}-1)^{n} \in K_{0}^{\widehat{\mu}}\left(\operatorname{Var}_{\mathbb{C}}\right)$. 


\subsection{The motivic nearby fiber}

Fix $\mathbb{K}=\mathbb{C}(t)$. Then the motivic nearby fiber is a ring homomorphism

$$
\begin{gathered}
\psi: K_{0}\left(\operatorname{Var}_{\mathbb{K}}\right) \rightarrow K_{0}^{\widehat{\mu}}\left(\operatorname{Var}_{\mathbb{C}}\right), \\
{[X] \mapsto \psi_{X} .}
\end{gathered}
$$

A result of Bittner [8] implies that $K_{0}\left(\operatorname{Var}_{\mathbb{K}}\right)$ is generated by the classes of smooth, proper varieties. In particular, the description below determines $\psi$.

We follow the description of the motivic nearby fiber in [56]. A variety $X$ over $\mathbb{K}$ is naturally interpreted as a complex variety with a morphism $f: X \rightarrow \mathbb{C}^{*} \backslash\left\{b_{1}, \ldots, b_{r}\right\}$, for some points $b_{1}, \ldots, b_{r}$. Assume that $X$ is smooth and extend $X$ to a variety $X^{\prime}$ with flat morphism $f: X^{\prime} \rightarrow \mathbb{A}^{1} \backslash\left\{b_{1}, \ldots, b_{r}\right\}$. After resolving singularities, we may assume that $X^{\prime}$ is smooth and that the central fiber $f^{-1}(0)=\sum_{i} m_{i} D_{i}$ is a simple normal crossings divisor with irreducible components $D_{1}, \ldots, D_{r}$, with multiplicities $m_{1}, \ldots, m_{r}$, respectively. For each nonempty subset $I \subseteq\{1, \ldots, r\}$, let $D_{I}^{\circ}=\cap_{i \in I} D_{i} \backslash \cup_{j \notin I} D_{j}$ and $m_{I}=\operatorname{gcd}\left(m_{i} \mid i \in I\right)$. Restrict $f$ to a small complex disk $\mathbb{D}$ about the origin and let $m$ be a common multiple of $m_{1}, \ldots, m_{r}$. Pullback $X^{\prime}$ via the map $\mathbb{D} \rightarrow \mathbb{D}, t \mapsto t^{m}$ and normalize to obtain a commutative diagram

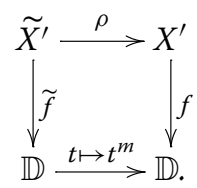

The action of $\mu_{m}$ on $\mathbb{D}$, with $\zeta \in \mu_{m}$ acting via $t \mapsto \zeta t$, extends to an action on $\widetilde{X}^{\prime}$, and hence a good $\widehat{\mu}$-action on the central fiber. Let $\widetilde{D_{I}^{\circ}}$ be proper transform of $D_{I}^{\circ}$ via $\rho$. Then $\rho: \widetilde{D_{I}^{\circ}} \rightarrow D_{I}^{\circ}$ is a $\mu_{m_{I}}$-covering. The motivic nearby fiber $\psi_{X} \in K_{0}^{\widehat{\mu}}\left(\operatorname{Var}_{\mathbb{C}}\right)$ of $X$ is given by

$$
\psi_{X}=\sum_{\emptyset \neq I \subseteq\{1, \ldots, r\}}\left[\widetilde{D}_{I}^{\circ} \circlearrowleft \widehat{\mu}\right](1-\mathbb{L})^{|I|-1} .
$$

\subsection{Motivic invariants}

The importance of the motivic nearby fiber comes from its specializations. In this section, we will explain the following commutative diagram, which will be crucial in the rest of the paper, where the first vertical map is the motivic nearby fiber:

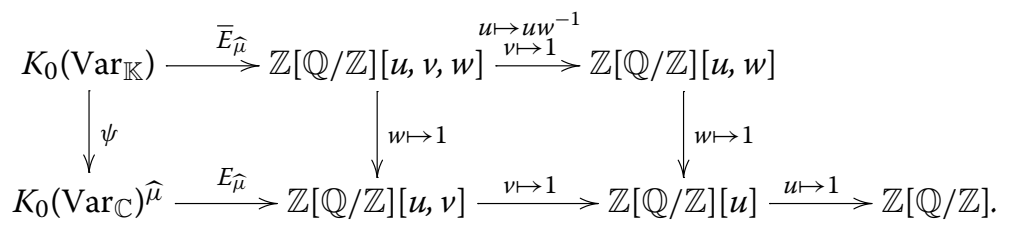

Throughout the paper, we will identify the group $\mathbb{Q} / \mathbb{Z}$ with the group $\mathbb{S}_{\mathbb{Q}}^{1}$ of rational points on the circle $\{z \in \mathbb{C}|| z \mid=1\}$, sending $[k] \in \mathbb{Q} / \mathbb{Z}$ to $\alpha=e^{2 \pi \sqrt{-1} k} \in \mathbb{S}_{\mathbb{Q}}^{1}$. We will consider the group algebra $\mathbb{Z}[\mathbb{Q} / \mathbb{Z}]$.

Remark 2.3 Consider the $\mathbb{Z}$-algebra involution on $\mathbb{Z}[\mathbb{Q} / \mathbb{Z}]$ defined by setting $\overline{[k]}=[-k]$, corresponding to complex conjugation on the circle. This extends coefficient-wise to an involution on a polynomial ring with coefficients in $\mathbb{Z}[\mathbb{Q} / \mathbb{Z}]$ that we will refer to as conjugation. 
Remark 2.4 Consider the natural $\mathbb{Z}$-algebra homomorphism $\mathbb{Z}[\mathbb{Q} / \mathbb{Z}] \rightarrow \mathbb{Z},[k] \mapsto 1$. One may apply this homomorphism below to get invariants with coefficients in $\mathbb{Z}$ rather than $\mathbb{Z}[\mathbb{Q} / \mathbb{Z}]$. The corresponding diagram of invariants above over $\mathbb{Z}$ is explained in detail in [30, Section 3].

Consider a complex vector space $B$ that admits a mixed Hodge structure [44] with corresponding vector space decomposition

$$
B \cong \bigoplus_{p, q} H^{p, q}(B) .
$$

Suppose the $\mu_{n}$ acts linearly on $B$, preserving the mixed Hodge structure. With the convention above, for $\alpha \in \mathbb{Q} / \mathbb{Z}$, we write $h^{p, q}(B)_{\alpha}$ for the dimension of the $\alpha$-eigenspace of $H^{p, q}(B)$, and write $h^{p, q}(B, \widehat{\mu}):=\sum_{\alpha \in \mathbb{Q} / \mathbb{Z}} h^{p, q}(B)_{\alpha} \alpha \in \mathbb{Z}[\mathbb{Q} / \mathbb{Z}]$. For a sequence of such representations $B_{\bullet}=\left\{B_{m} \mid m \geq 0\right\}$, set $e^{p, q}\left(B_{\bullet}, \widehat{\mu}\right)=\sum_{m}(-1)^{m} h^{p, q}\left(B_{m}, \widehat{\mu}\right) \in \mathbb{Z}[\mathbb{Q} / \mathbb{Z}]$. Then the equivariant Hodge polynomial of $B_{\bullet}$ is defined by

$$
E\left(B_{\bullet}\right)=E\left(B_{\bullet} ; u, v\right)=\sum_{p, q} e^{p, q}\left(B_{\bullet}, \widehat{\mu}\right) u^{p} v^{q} \in \mathbb{Z}[\mathbb{Q} / \mathbb{Z}][u, v] .
$$

In [15], Deligne proved that the $m^{\text {th }}$ cohomology group with compact supports $H_{c}^{m}(V)$ of a complex variety $V$ admits a canonical mixed Hodge structure with decreasing filtration $F^{\bullet}$ called the Hodge filtration and increasing filtration $W_{\bullet}$ called the Deligne weight filtration. Given a good action of $\widehat{\mu}$ on $V$, we may set $B_{m}=H^{p, q}\left(H_{c}^{m}(V)\right)$ above. The corresponding equivariant Hodge polynomial is denoted $E_{\widehat{\mu}}(V ; u, v) \in \mathbb{Z}[\mathbb{Q} / \mathbb{Z}][u, v]$, and is called the equivariant Hodge-Deligne polynomial of $V$. The invariants $h^{p, q}\left(H_{c}^{m}(V)\right)_{\alpha}$ are called the equivariant mixed Hodge numbers of $V$. We have a ring homomorphism called the equivariant Hodge-Deligne map:

$$
E_{\widehat{\mu}}: K_{0}^{\widehat{\mu}}\left(\operatorname{Var}_{k}\right) \rightarrow \mathbb{Z}[\mathbb{Q} / \mathbb{Z}][u, v],[V] \mapsto E_{\widehat{\mu}}(V ; u, v) .
$$

As in Remark 2.4, we will also consider the corresponding invariants over $\mathbb{Z}$. In this case, we have the usual mixed Hodge numbers $h^{p, q}\left(H_{c}^{m}(V)\right)=\sum_{\alpha} h^{p, q}\left(H_{c}^{m}(V)\right)_{\alpha}$ and corresponding Hodge-Deligne polynomial $E(V ; u, v) \in \mathbb{Z}[u, v]$.

Remark 2.5 More generally, for any finite group $G$ acting algebraically on a complex variety $V$, we obtain a linear action of $G$ on $H^{p, q}\left(H_{c}^{m}(V)\right)$, and one can form the equivariant Hodge-Deligne polynomial

$$
E_{G}(V ; u, v)=\sum_{p, q} \sum_{m}(-1)^{m} H^{p, q}\left(H_{c}^{m}(V)\right) u^{p} v^{q} \in R(G)[u, v],
$$

where $R(G)$ is the complex representation ring of $G$. This induces a ring homomorphism $\widetilde{K}_{0}^{G}\left(\operatorname{Var}_{\mathbb{C}}\right) \rightarrow R(G)[u, v]$. This invariant was introduced and studied by the author in [54], generalizing the notion of weight polynomial due to Dimca and Lehrer [19], and the notion of equivariant $\chi_{y}$-genus due to Cappell, Maxim and Shaneson [10]. In our case, $\mathbb{Z}[\mathbb{Q} / \mathbb{Z}]$ may be viewed as the direct limit associated with the restriction maps $R\left(\mu_{n}\right) \rightarrow R\left(\mu_{n d}\right)$ induced by the epimorphisms $\mu_{n d} \rightarrow \mu_{n}, \zeta \mapsto \zeta^{d}$.

Recall that a variety $X$ over $\mathbb{K}$ is naturally interpreted as a complex variety with a morphism $f: X \rightarrow \mathbb{C}^{*} \backslash\left\{b_{1}, \ldots, b_{r}\right\}$, for some points $b_{1}, \ldots, b_{r}$. Restricting $X$ to a family over a sufficiently small punctured complex disk centered at the origin, and fixing a fiber $X_{\text {gen }}:=f^{-1}(t)$ and counter-clockwise orientation around the disk, there exists 
a quasi-unipotent monodromy map $T=T_{s} T_{u}: H_{c}^{m}\left(X_{\text {gen }} ; \mathbb{C}\right) \rightarrow H_{c}^{m}\left(X_{\text {gen }} ; \mathbb{C}\right)$, where $T_{s}$ is semi-simple and $T_{u}$ is unipotent. Then the cohomology groups $H_{c}^{m}\left(X_{\mathrm{gen}}\right)$ admit a weight filtration $M_{\bullet}$ called the monodromy weight filtration, and $T_{s}$ acts preserving the filtrations $\left(F^{\bullet}, M_{\bullet}, W_{\bullet}\right)$. We will write $H_{c}^{m}\left(X_{\infty}\right)$ to denote $H_{c}^{m}\left(X_{\text {gen }}\right)$ with the filtrations $\left(F^{\bullet}, M_{\bullet}, W_{\bullet}\right)$. The filtrations $\left(F^{\bullet}, M_{\bullet}\right)$ induce a mixed Hodge structure on $H_{c}^{m}\left(X_{\infty}\right)$, and the nilpotent operator $N=\log T_{u}$ is a morphism of mixed Hodge structures of type $(-1,-1)$. The corresponding invariants $h^{p, q}\left(H_{c}^{m}\left(X_{\infty}\right)\right)_{\alpha}$ are called the equivariant limit mixed Hodge numbers, and the corresponding equivariant Hodge polynomial $E\left(X_{\infty}, \widehat{\mu} ; u, v\right) \in \mathbb{Z}[\mathbb{Q} / \mathbb{Z}][u, v]$ is called equivariant limit Hodge-Deligne polynomial. A deep result of Denef and Loeser states that this is a specialization of the motivic nearby fiber in the sense that $E\left(X_{\infty}, \widehat{\mu} ; u, v\right)=E_{\widehat{\mu}}\left(\psi_{X}\right)$. As in Remark 2.4, we may consider the corresponding invariants over $\mathbb{Z}$, i.e., the limit mixed Hodge numbers and limit HodgeDeligne polynomial.

Before proceeding, we recall the following standard linear algebra construction.

Definition 2.6 Let $A$ be a nilpotent linear operator on a finite-dimensional vector space $V$ such that $A^{r+1}=0$. Then the $A$-weight filtration centered at $r$ is the increasing filtration $\left\{V_{\bullet}\right\}$ of $V$ by subspaces

$$
0 \subseteq V_{0} \subseteq V_{1} \subseteq \cdots \subseteq V_{2 r}=V
$$

uniquely determined by

(1) $A\left(V_{k}\right) \subseteq V_{k-2}$,

(2) the induced map $A^{k}: G r_{r+k} V \rightarrow G r_{r-k} V$ is an isomorphism,

for any nonnegative integer $k$. Here we set $V_{k}=0$ for $k<0$. The filtration encodes the Jordan block structure of $A$. Explicitly, for $1 \leq k \leq r+1$, the number of Jordan blocks of size $k$ equals

$$
\operatorname{dim} G r_{r+1-k} V-\operatorname{dim} G r_{r-1-k} V .
$$

The monodromy weight filtration $M_{\bullet}$ has the following important geometric property: for every nonnegative integer $r$, the induced filtration $M(r)$. on $G r_{r}^{W} H_{c}^{m}\left(X_{\infty}\right)$ coincides with the $N(r)$-weight filtration centered at $r$, where $N(r)$ is the nilpotent operator induced by $N=\log T_{u}$. It follows from Definition 2.6 that the monodromy weight filtration, together with the action of $T_{s}$, encodes the Jordan block structure of the monodromy operator $T$ acting on $G r_{r}^{W} H_{c}^{m}\left(X_{\infty}\right)$. Moreover, $\left(F^{\bullet}, M_{\bullet}\right)$ induces a mixed Hodge structure on $G_{r}^{W} H_{c}^{m}\left(X_{\infty}\right)$ that is invariant under $T_{s}$, and $N(r)$ is a morphism of mixed Hodge structures of type $(-1,-1)$. The associated equivariant mixed Hodge numbers

$$
h^{p, q}\left(G r_{r}^{W} H_{c}^{m}\left(X_{\infty}\right)\right)_{\alpha}=\left(G r_{F}^{p} G r_{p+q}^{M} G r_{r}^{W} H_{c}^{m}\left(X_{\infty}\right)\right)_{\alpha}
$$

are called the equivariant refined limit mixed Hodge numbers, and the associated equivariant Hodge polynomial is the coefficient of $w^{r}$ in a polynomial $E\left(X_{\infty}, \widehat{\mu} ; u, v, w\right) \in$ $\mathbb{Z}[\mathbb{Q} / \mathbb{Z}][u, v, w]$ called the equivariant refined limit Hodge-Deligne polynomial. As in Remark 2.4, we have corresponding invariants over $\mathbb{Z}$ that were introduced in [30, Section 3], to where we refer the reader for further details. We have a ring homomorphism

$$
\begin{gathered}
\bar{E}_{\widehat{\mu}}: K_{0}\left(\operatorname{Var}_{\mathbb{K}}\right) \rightarrow \mathbb{Z}[\mathbb{Q} / \mathbb{Z}][u, v, w], \\
{[X] \mapsto E\left(X_{\infty}, \widehat{\mu} ; u, v, w\right) .}
\end{gathered}
$$


It follows from the definitions that we recover the equivariant limit mixed Hodge numbers and the usual mixed Hodge numbers of $X_{\text {gen }}$ via the specializations:

$$
\begin{aligned}
h^{p, q}\left(H_{c}^{m}\left(X_{\infty}\right)\right)_{\alpha} & =\sum_{r} h^{p, q, r}\left(H_{c}^{m}\left(X_{\infty}\right)\right)_{\alpha}, \\
h^{p, r-p}\left(H_{c}^{m}\left(X_{\text {gen }}\right)\right) & =\sum_{q} \sum_{\alpha \in \mathbb{Q} / \mathbb{Z}} h^{p, q, r}\left(H_{c}^{m}\left(X_{\infty}\right)\right)_{\alpha} .
\end{aligned}
$$

Correspondingly, we have $E\left(X_{\infty}, \widehat{\mu} ; u, v, 1\right)=E\left(X_{\infty}, \widehat{\mu} ; u, v\right)$, and $E\left(X_{\infty}, \widehat{\mu} ; u w^{-1}, 1, w\right)$ restricts to the Hodge-Deligne polynomial $E\left(X_{\text {gen }} ; u, w\right) \in \mathbb{Z}[u, w]$. The further specializations $E\left(X_{\text {gen }} ; u, 1\right)$ and $E\left(X_{\text {gen }} ; 1,1\right)$ are the $\chi_{y}$-characteristic and Euler characteristic of $X_{\text {gen }}$, respectively.

Remark 2.7 With the notation above, since $N(r)$ is a morphism of mixed Hodge structures of type $(-1,-1)$, the isomorphisms (2) in Definition 2.6 imply that for $0 \leq j \leq r$, the sequence $\left\{h^{i+j, i, r}\left(H_{c}^{m}\left(X_{\infty}\right)\right)_{\alpha} \mid 0 \leq i \leq r-j\right\}$ is symmetric and unimodal.

Remark 2.8 Since $H^{p, q}\left(H_{c}^{m}\left(X_{\infty}\right)\right)$ and $H^{q, p}\left(H_{c}^{m}\left(X_{\infty}\right)\right)$ are conjugate, and by Remark 2.7, the refined limit mixed Hodge numbers satisfy the following symmetries:

$$
\begin{aligned}
h^{p, q, r}\left(H_{c}^{m}\left(X_{\infty}\right)\right)_{\alpha} & =h^{r-q, r-p, r}\left(H_{c}^{m}\left(X_{\infty}\right)\right)_{\alpha}=h^{q, p, r}\left(H_{c}^{m}\left(X_{\infty}\right)\right)_{\alpha^{-1}} \\
& =h^{r-p, r-q, r}\left(H_{c}^{m}\left(X_{\infty}\right)\right)_{\alpha^{-1}} .
\end{aligned}
$$

Hence the equivariant refined limit Hodge-Deligne polynomial satisfies the following symmetries with respect to the conjugation action on $\mathbb{Z}[\mathbb{Q} / \mathbb{Z}][u, v, w]$ described in Remark 2.3:

$$
\begin{aligned}
& E\left(X_{\infty}, \widehat{\mu} ; u, v, w\right)=\overline{E\left(X_{\infty}, \widehat{\mu} ; v, u, w\right)}, \\
& E\left(X_{\infty}, \widehat{\mu} ; u, v, w\right)=\overline{E\left(X_{\infty}, \widehat{\mu} ; u^{-1}, v^{-1}, u v w\right)} .
\end{aligned}
$$

Example 2.9 If $V$ is a complex variety and $X=V \times_{\mathbb{C}} \mathbb{K}$, then $X$ may be regarded as a trivial family over $\mathbb{D}^{*}$. In this case, $N$ is identically zero, $M_{\bullet}$ coincides with the Deligne filtration $W_{\bullet}$, and $E\left(X_{\infty}, \widehat{\mu} ; u, v, w\right)=E(V ; u w, v w)$.

Example 2.10 If $X$ is smooth and proper, then $\operatorname{Gr}_{r}^{W} H^{m}\left(X_{\text {gen }}\right)=0$ unless $m=r$. In this case, the monodromy weight filtration, together with the action of $T_{s}$, encodes the Jordan block decomposition of $T$. In this case,

$$
E\left(X_{\infty}, \widehat{\mu} ; u, v, w\right)=\sum_{p, q, m}(-1)^{m} h^{p, q}\left(H^{m}\left(X_{\infty}\right)\right)_{\alpha} u^{p} v^{q} w^{m} \alpha .
$$

\section{Tropical geometry}

In this section we prove our formula for the motivic nearby fiber. The nonequivariant version of this result is [30, Theorem 1.2]. We continue with the notation of the previous section. In particular, $\mathbb{K}=\mathbb{C}(t)$.

Let $w=\left(w_{1}, \ldots, w_{n}\right) \in \mathbb{R}^{n}$ and let $f \in \mathbb{K}\left[x_{1}^{ \pm 1}, \ldots, x_{n}^{ \pm 1}\right]$. Then the initial degeneration $\operatorname{in}_{w}(f) \in \mathbb{C}\left[x_{1}^{ \pm 1}, \ldots, x_{n}^{ \pm 1}\right]$ of $f$ with respect to $w$ is defined as follows: write $f=\sum_{u \in \mathbb{Z}^{n}} \lambda_{u} t^{\omega_{f}(u)} g_{u}(t) x^{\alpha}$ for some $\lambda_{u} \in \mathbb{C}, \omega_{f}(u) \in \mathbb{Z}$ and $g_{u}(t) \in \mathbb{C}(t)$ such that $g(1)=1$, and let $m_{f}=\min \left\{\omega_{f}(u)+w \cdot u \mid \lambda_{u} \neq 0\right\}$. Then

$$
\operatorname{in}_{w}(f)=\sum_{\substack{u \in \mathbb{Z}^{n} \\ \omega_{f}(u)+w \cdot u=m_{f}}} \lambda_{u} x^{u} .
$$


Assume that $w$ is rational and consider the element

$$
\exp (2 \pi \sqrt{-1} w):=\left(e^{2 \pi \sqrt{-1} w_{1}}, \ldots, e^{2 \pi \sqrt{-1} w_{n}}\right) \in\left(\mathbb{C}^{*}\right)^{n} .
$$

Then multiplication by $\exp (2 \pi \sqrt{-1} w)$ induces a finite-order automorphism $\beta_{w}$ of $\left(\mathbb{C}^{*}\right)^{n}$ and hence a good action of $\widehat{\mu}$ on $\left(\mathbb{C}^{*}\right)^{n}$. Note that if $w \neq 0$, then $\beta_{w}$ acts freely on $\left(\mathbb{C}^{*}\right)^{n}$. We compute

$$
\left(\beta_{w}\right)^{*}\left(\operatorname{in}_{w}(f)\right)=\operatorname{in}_{w}(f)\left(e^{2 \pi \sqrt{-1} w_{1}} x_{1}, \ldots, e^{2 \pi \sqrt{-1} w_{1}} x_{n}\right)=e^{2 \pi \sqrt{-1} m_{f}} \operatorname{in}_{w}(f) .
$$

Let $X^{\circ} \subseteq\left(\mathbb{K}^{*}\right)^{n}$ be a closed subvariety defined by an ideal $I \subseteq \mathbb{K}\left[x_{1}^{ \pm 1}, \ldots, x_{n}^{ \pm 1}\right]$. Then the initial degeneration $\operatorname{in}_{w} X^{\circ}$ is the closed subscheme of $\left(\mathbb{C}^{*}\right)^{n}$ defined by the ideal $\operatorname{in}_{w} I:=\left(\operatorname{in}_{w}(f) \mid f \in I\right) \subseteq \mathbb{C}\left[x_{1}^{ \pm 1}, \ldots, x_{n}^{ \pm 1}\right]$. By (4), $\operatorname{in}_{w} I$ is invariant under $\beta_{w}$, and hence we have an induced action on $\operatorname{in}_{w} X^{\circ}$.

A variety $X$ over $\mathbb{K}$ is very affine if it admits a closed embedding $X \subseteq\left(\mathbb{K}^{*}\right)^{n}$ for some $n$. Tevelev introduced the notion of a schön, very affine variety. A closed subvariety $X^{\circ} \subseteq\left(\mathbb{K}^{*}\right)^{n}$ is schön if $\operatorname{in}_{w} X^{\circ} \subseteq\left(\mathbb{C}^{*}\right)^{n}$ is a smooth (reduced) subvariety for every $w \in \mathbb{R}^{n}$ [25, Prop 3.9]. A very affine variety $X$ over $\mathbb{K}$ is schön if it admits a schön closed embedding $X \subseteq\left(\mathbb{K}^{*}\right)^{n}$ for some $n$ (cf. [37, Lemma 2.11]). Note that a complex variety $V$ may be viewed via base change as a variety over $\mathbb{K}$, and hence we may consider the same notions. The definition of schön for a hypersurface of a complex torus agrees with the notion of nondegeneracy with respect to the Newton polytope [33].

The tropical variety $\operatorname{Trop}\left(X^{\circ}\right)$ associated with $X^{\circ} \subseteq\left(\mathbb{K}^{*}\right)^{n}$ is the set of points

$$
\left\{w \in \mathbb{R}^{n} \mid \operatorname{in}_{w} X^{\circ} \neq \emptyset\right\} .
$$

The tropical variety $\operatorname{Trop}\left(X^{\circ}\right)$ can be given a rational polyhedral structure $\Sigma$ such that initial degeneration at $w \in \operatorname{Trop}\left(X^{\circ}\right)$ only depends on the cell containing $w$ in its relative interior (this follows from [37, Theorem 1.5]). Hence for every cell $\gamma$ of $\Sigma$, we may define $\operatorname{in}_{\gamma} X^{\circ}:=\operatorname{in}_{w} X^{\circ}$ for any $w \in \mathbb{R}^{n}$ in the relative interior of $\gamma$. Moreover, by fixing such a $w \in \mathbb{Q}^{n}$, we obtain a good action of $\widehat{\mu}$ on $\operatorname{in}_{\gamma} X^{\circ}$ via multiplication by $\exp (2 \pi \sqrt{-1} w)$.

Example 3.1 Consider the variety $X^{\circ}=\left\{\sum_{i=1}^{n} x_{i}^{m_{i}}=t^{e}\right\} \subseteq\left(\mathbb{K}^{*}\right)^{n}$, for some $m_{i} \in \mathbb{Z}_{>0}$ and $e \in \mathbb{Z}$. Let $e_{1}, \ldots, e_{n}$ denote the standard basis vectors of $\mathbb{R}^{n}$. Then $X^{\circ} \subseteq\left(\mathbb{K}^{*}\right)^{n}$ is schön and $\operatorname{Trop}\left(X^{\circ}\right) \subseteq \mathbb{R}^{n}$ is the affine translation by $\left(e / m_{1}, \ldots, e / m_{n}\right)$ of the $(n-1)$-dimensional fan with cones spanned by subsets of size at most $n-1$ of $\left\{e_{1}, \ldots, e_{n},-e_{1} / m_{1}-\cdots-e_{n} / m_{n}\right\}$. Let $w=\left(e / m_{1}, \ldots, e / m_{n}\right)$. Then $\widehat{\mu}$ acts on $\operatorname{in}_{w} X^{\circ}=\left\{\sum_{i=1}^{n} x_{i}^{m_{i}}=1\right\} \subseteq\left(\mathbb{C}^{*}\right)^{n}$ via multiplication by $\left(e^{2 \pi \sqrt{-1} e / m_{1}}, \ldots, e^{2 \pi \sqrt{-1} e / m_{n}}\right)$. Considering $X^{\circ}$ as a family $f: X^{\circ} \rightarrow \mathbb{A}^{1}$, for $0 \leq a \leq 1$, multiplication by $\left(e^{2 \pi \sqrt{-1} a e / m_{1}}, \ldots, e^{2 \pi \sqrt{-1} a e / m_{n}}\right)$ induces an isomorphism between $f^{-1}(1)$ and $f^{-1}\left(e^{2 \pi \sqrt{-1} a}\right)$. We deduce that the action of $\widehat{\mu}$ coincides with the action of monodromy induced by moving anti-clockwise around the unit circle (more generally, see Example 5.6).

Recall that our goal is to compute the motivic nearby fiber defined in Sect. 2.2:

$$
\begin{gathered}
\psi: K_{0}\left(\operatorname{Var}_{\mathbb{K}}\right) \rightarrow K_{0}^{\widehat{\mu}}\left(\operatorname{Var}_{\mathbb{C}}\right), \\
{[X] \mapsto \psi_{X} .}
\end{gathered}
$$

A result of Luxton and $\mathrm{Qu}$ [37, Theorem 6.11] that was conjectured by Tevelev in [59], implies that every variety over $\mathbb{K}$ admits a stratification into locally closed, very affine, 
schön subvarieties. By additivity property $(1)$ of $K_{0}\left(\operatorname{Var}_{\mathbb{K}}\right)$, it follows that we may reduce the computation of $\psi$ to the case when $X^{\circ} \subseteq\left(\mathbb{K}^{*}\right)^{n}$ is a schön subvariety. In this case, we have the following equivariant generalization of [30, Theorem 1.2].

Theorem 3.2 Let $X^{\circ} \subseteq\left(\mathbb{K}^{*}\right)^{n}$ be a schön closed subvariety and let $\Sigma$ be a rational polyhedral structure on $\operatorname{Trop}\left(X^{\circ}\right)$. Then the motivic nearby fiber $\psi_{X^{\circ}} \in K_{0}^{\widehat{\mu}}\left(\operatorname{Var}_{\mathbb{C}}\right)$ is given by

$$
\psi_{X^{\circ}}=\sum_{\substack{\gamma \in \Sigma \\ \gamma \text { bounded }}}(-1)^{\operatorname{dim} \gamma}\left[\operatorname{in}_{\gamma} X^{\circ} \circlearrowleft \widehat{\mu}\right],
$$

where the action of $\widehat{\mu}$ on $\operatorname{in}_{\gamma} X^{\circ} \subseteq\left(\mathbb{C}^{*}\right)^{n}$ is induced by multiplication by $\exp (2 \pi \sqrt{-1} w)=$ $\left(e^{2 \pi \sqrt{-1} w_{1}}, \ldots, e^{2 \pi \sqrt{-1} w_{n}}\right)$ for a fixed choice of $w \in \mathbb{Q}^{n}$ in the relative interior of $\gamma$.

Proof We first recall the relevant geometric setting, as described in [25, Sections 1,2]. We refer the reader to [24] for the appropriate background on toric geometry.

Let $N=\mathbb{Z}^{n}$, and for each cell $\gamma \in \Sigma$, let $C_{\gamma}$ denote the cone generated by $\gamma \times\{1\}$ in $N_{\mathbb{R}} \times \mathbb{R}$. The cones $C_{\gamma}$ form a fan $\widetilde{\Sigma}$ in $N_{\mathbb{R}} \times \mathbb{R}$, and the recession fan $\Delta$ is defined to be the intersection of $\widetilde{\Sigma}$ with $N_{\mathbb{R}} \times\{0\}$. Projection pr $: N_{\mathbb{R}} \times \mathbb{R} \rightarrow \mathbb{R}$ onto the second coordinate induces a morphism of fans $\widetilde{\Sigma} \rightarrow \mathbb{R}_{\geq 0}$. Fix a positive integer $m$ such that $m \Sigma$ has a lattice polyhedral structure, and let $\widetilde{\mathbb{P}}$ and $\mathbb{P}$ denote the complex toric varieties corresponding to the fan $\widetilde{\Sigma}$ with respect to the lattices $N \times m \mathbb{Z}$ and $N \times \mathbb{Z}$, respectively. We have an induced commutative diagram corresponding to pulling back and normalizing $\mathbb{P}$ :

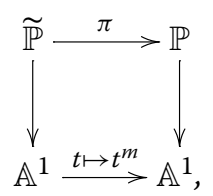

where the vertical arrows are flat. After removing the closed torus invariant divisors corresponding to nonzero cones in $\Delta$, the nonzero fibers of $\widetilde{\mathbb{P}}$ and $\mathbb{P}$ are tori and the central fibers have decompositions into disjoint unions of tori:

$$
\widetilde{\mathbb{P}}^{\circ}=\bigcup_{\substack{\gamma \in \Sigma \\ \gamma \text { bounded }}} \widetilde{U}_{\gamma}, \quad \mathbb{P}^{\circ}=\bigcup_{\substack{\gamma \in \Sigma \\ \gamma \text { bounded }}} U_{\gamma} .
$$

Let $\pi_{\gamma}: \widetilde{U}_{\gamma} \rightarrow U_{\gamma}$ denote the morphism of tori induced by $\pi$. This has the following explicit description. Let $L_{\gamma}^{\prime}$ denote the linear span of $C_{\gamma}$, and let $L_{\gamma}=L_{\gamma}^{\prime} \cap\left(N_{\mathbb{R}} \times\{0\}\right) \subseteq N_{\mathbb{R}}$. That is, $L_{\gamma}$ is the translate of the affine span of $\gamma$ to the origin in $N_{\mathbb{R}}$. Fix $m_{\gamma} \in \mathbb{Z}_{>0}$ such that $\operatorname{pr}\left((N \times \mathbb{Z}) \cap L_{\gamma}^{\prime}\right)=m_{\gamma} \mathbb{Z}$. Let $N_{\gamma}=N / N \cap L_{\gamma}$ with distinguished rational point $[\gamma] \in$ $\left(N_{\gamma}\right)_{\mathbb{Q}}$. Then projection along $L_{\gamma}^{\prime}$ induces an isomorphism $N_{\mathbb{Q}} \times \mathbb{Q} /\left(N_{\mathbb{Q}} \times \mathbb{Q}\right) \cap L_{\gamma}^{\prime} \rightarrow\left(N_{\gamma}\right)_{\mathbb{Q}}$ that sends $(0,1)=([-\gamma], 0)+([\gamma], 1) \mapsto[-\gamma]$. This isomorphism induces the vertical maps in the following commutative diagrams:

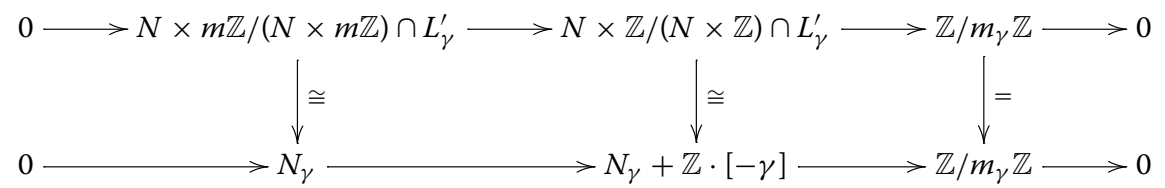

Applying the functors $\operatorname{Hom}(\mathbb{Z})$ and then $\operatorname{Hom}\left(, \mathbb{C}^{*}\right)$ induces

$$
1 \rightarrow \mu_{m_{\gamma}} \rightarrow \widetilde{U}_{\gamma} \stackrel{\pi_{\gamma}}{\rightarrow} U_{\gamma} \rightarrow 1
$$


Letting $M=\operatorname{Hom}(N, \mathbb{Z})$ and $M_{\gamma}=\operatorname{Hom}\left(N_{\gamma}, \mathbb{Z}\right)$, we identify $\widetilde{U}_{\gamma}=\operatorname{Hom}\left(M_{\gamma}, \mathbb{C}^{*}\right)$. Then the above sequence says that the rational point $[-\gamma] \in\left(N_{\gamma}\right)_{\mathbb{Q}}$ corresponds to an element $\exp (2 \pi \sqrt{-1}[-\gamma]) \in \widetilde{U}_{\gamma}$ of order $m_{\gamma}$ such that $U_{\gamma}$ is the quotient of $\widetilde{U}_{\gamma}$ by the action of multiplication by $\exp (2 \pi \sqrt{-1}[-\gamma])$. In what follows, we let $T=\operatorname{Hom}\left(M, \mathbb{C}^{*}\right)$ and consider the multiplication map $T \times \widetilde{U}_{\gamma} \rightarrow \widetilde{U}_{\gamma}$. We also fix the distinguished element $p \in \widetilde{U}_{\gamma}=\operatorname{Hom}\left(M_{\gamma}, \mathbb{C}^{*}\right)$ corresponding to the constant map 1.

Recall that $X^{\circ}$ may viewed as a family of complex varieties over $\mathbb{C}^{*} \backslash\left\{b_{1}, \ldots, b_{r}\right\}$, for some points $b_{1}, \ldots, b_{r}$. Let $\mathbb{D}$ be a sufficiently small complex disk centered at the origin, and restrict $X^{\circ}$ to a family over the punctured disk $\mathbb{D}^{*}$, and $\widetilde{\mathbb{P}}$ and $\mathbb{P}$ to families over $\mathbb{D}$. Taking the closure $\mathcal{X}$ of $X^{\circ}$ in $\mathbb{P}$ induces a commutative diagram:

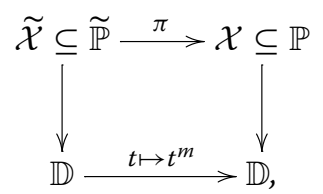

where for each bounded cell $\gamma \in \Sigma$, we have a corresponding $\mu_{m_{\gamma}}$-covering $\widetilde{V}_{\gamma}^{\circ}:=\widetilde{\mathcal{X}} \cap$ $\widetilde{U}_{\gamma} \rightarrow V_{\gamma}^{\circ}:=\mathcal{X} \cap U_{\gamma}$ corresponding to the action of multiplication by $\exp (2 \pi \sqrt{-1}[-\gamma])$. As above, consider the induced multiplication map $m: T \times \widetilde{V}_{\gamma}^{\circ} \rightarrow \widetilde{U}_{\gamma}$ and consider the fiber $m^{-1}(p)$. By [25, p. 8], projection onto $T$ identifies $m^{-1}(p)$ with $\operatorname{in}_{\gamma} X^{\circ}$, while projection onto $\widetilde{V}_{\gamma}^{\circ}$ identifies $m^{-1}(p)$ with $T_{\gamma} \times \widetilde{V}_{\gamma}^{\circ}$, where $T_{\gamma}$ is the subtorus that fixes $U_{\gamma}$ pointwise. Fix a rational point $w \in N_{\mathbb{Q}}$ in the relative interior of $\gamma$, and consider the corresponding point $\exp (2 \pi \sqrt{-1} w) \in T$ of finite order. Then multiplication by $\left(\exp (2 \pi \sqrt{-1} w), \exp (2 \pi \sqrt{-1}[-\gamma])\right.$ preserves $m^{-1}(p)$, and, using Example 2.2, we conclude that

$$
\left[\operatorname{in}_{\gamma} X^{\circ} \circlearrowleft \widehat{\mu}\right]=\left[\tilde{V}_{\gamma}^{\circ} \circlearrowleft \widehat{\mu}\right](\mathbb{L}-1)^{\operatorname{dim} \gamma},
$$

where $\widehat{\mu}$ acts via multiplication by $\exp (2 \pi \sqrt{-1} w)$ and $\exp (2 \pi \sqrt{-1}[-\gamma])$, respectively. In particular, this shows that the left-hand side is independent of the choice of $w$ in the relative interior of $\gamma$.

We now complete the proof. We first verify that the right-hand side of the statement in the theorem is independent of the choice of polyhedral structure $\Sigma$. Indeed, if $\Sigma^{\prime}$ is a polyhedral structure refining $\Sigma$, then, using the independence of the choice of $w$ above

$$
\begin{aligned}
& \sum_{\substack{\gamma^{\prime} \in \Sigma^{\prime} \\
\gamma \text { bounded }}}(-1)^{\operatorname{dim} \gamma^{\prime}}\left[\operatorname{in}_{\gamma^{\prime}} X^{\circ} \circlearrowleft \widehat{\mu}\right] \\
& =\sum_{\gamma \in \Sigma}(-1)^{\operatorname{dim} \gamma}\left[\operatorname{in}_{\gamma} X^{\circ} \circlearrowleft \widehat{\mu}\right]\left(\sum_{\substack{\gamma^{\prime} \in \Sigma^{\prime}, \gamma^{\prime} \text { bounded } \\
\operatorname{Int}\left(\gamma^{\prime}\right) \subseteq \operatorname{Int}(\gamma)}}(-1)^{\operatorname{dim} \gamma-\operatorname{dim} \gamma^{\prime}}\right) .
\end{aligned}
$$

Hence, it is enough to show that

$$
\sum_{\substack{\gamma^{\prime} \in \Sigma^{\prime}, \gamma^{\prime} \text { bounded, } \\
\operatorname{Int}\left(\gamma^{\prime}\right) \subseteq \operatorname{Int}(\gamma)}}(-1)^{\operatorname{dim} \gamma^{\prime}}=\left\{\begin{array}{cl}
(-1)^{\operatorname{dim} \gamma} & \text { if } \gamma \text { is bounded } \\
0 & \text { otherwise. }
\end{array}\right.
$$

Topological and combinatorial proofs of the above fact are given in the proofs of [30, Lemma 2.2] and [28, Theorem 3.4], respectively. 
After possibly replacing $\widetilde{\Sigma}$ with a smooth fan refinement, and replacing $\Sigma$ with $\widetilde{\Sigma} \cap\left(N_{\mathbb{R}} \times\right.$ $\{1\})$, we may assume that $\mathbb{P}$ is smooth and the central fiber $\mathcal{X}_{0}$ is a simple normal crossings divisor. Let $\left\{v_{1}, \ldots, v_{s}\right\}$ denote the vertices of $\gamma$. Then $\mathcal{X}_{0}$ has irreducible components $\left\{D_{1}, \ldots, D_{s}\right\}$ with multiplicities $\left\{m_{1}, \ldots, m_{s}\right\}$, respectively, where $m_{i}$ is the smallest positive integer $m$ such that $m v_{i} \in N$. For every nonempty subset $I \subseteq\{1, \ldots s\}$, let $\gamma_{I}$ be the bounded cell of $\Sigma$ given by the convex hull of $\left\{v_{i} \mid i \in I\right\}$. Observe that every bounded cell appears in this way. As above, $D_{I}^{\circ}=\cap_{i \in I} D_{i} \backslash \cup_{j \notin I} D_{j}=V_{\gamma}^{\circ}$ admits an $\mu_{m_{\gamma}}$-covering $\widetilde{V}_{\gamma}^{\circ} \rightarrow V_{\gamma}^{\circ}$ corresponding to the action of multiplication by $\exp (2 \pi \sqrt{-1}[-\gamma])$. It follows from the fact that $C_{\gamma}$ is a smooth cone in $N \times \mathbb{Z}$ that $m_{\gamma}=\operatorname{gcd}\left(m_{i} \mid i \in I\right)$. The result now follows from (5) above together with the definition of the motivic nearby fiber given in (2).

Remark 3.3 It is a corollary of the proof above that in the statement of Theorem 3.2, one may consider the action on $\operatorname{in}_{\gamma} X^{\circ} \subseteq\left(\mathbb{C}^{*}\right)^{n}$ to be induced by multiplication by $\exp (2 \pi \sqrt{-1} w)$ for any fixed choice of $w \in \mathbb{Q}^{n}$ in the affine span of $\gamma$.

We will see interesting examples of the above theorem in Sect. 5 and Sect. 6. For the moment, we have the following simple example.

Example 3.4 Following on with Example 3.1, let $X^{\circ}=\left\{\sum_{i=1}^{n} x_{i}^{m_{i}}=t^{e}\right\} \subseteq\left(\mathbb{K}^{*}\right)^{n}$ for some $m_{i} \in \mathbb{Z}_{>0}$ and $e \in \mathbb{Z}$. Then $\psi_{X^{\circ}}=\left[\left\{\sum_{i=1}^{n} x_{i}^{m_{i}}=1\right\} \subseteq\left(\mathbb{C}^{*}\right)^{n} \circlearrowleft \widehat{\mu}\right]$, with $\widehat{\mu}$-action induced by multiplication by $\left(e^{2 \pi \sqrt{-1} e / m_{1}}, \ldots, e^{2 \pi \sqrt{-1} e / m_{n}}\right)$. If $X=\left\{\sum_{i=1}^{n} x_{i}^{m_{i}}=t^{e}\right\} \subseteq \mathbb{K}^{n}$ denotes the closure of $X^{\circ}$ in $\mathbb{K}^{n}$, then $\psi_{X}=\left[\left\{\sum_{i=1}^{n} x_{i}^{m_{i}}=1\right\} \subseteq \mathbb{C}^{n} \circlearrowleft \widehat{\mu}\right]$, with action again given by multiplication by $\left(e^{2 \pi \sqrt{-1} e / m_{1}}, \ldots, e^{2 \pi \sqrt{-1} e / m_{n}}\right)$. As in Example 3.1, the above action coincides with the action induced by monodromy.

\section{The combinatorics of height functions}

In this section, we present the relevant combinatorics of this paper. Weighted Ehrhart theory was introduced by the author in [52] both in order to extend and reprove many results in Ehrhart theory, the study of enumeration of lattice points in polytopes, and in order to give explicit computations of motivic integrals on toric stacks [53]. We present a generalization of this theory below using an extension of Stanley's theory of subdivisions [51] presented in [29]. For brevity, we will only present the relevant results and refer the reader to [29] and [52] for details of proofs.

\subsection{Eulerian posets}

We first recall some basic notions on Eulerian posets. Consider a finite poset $B$ containing a minimal element $\widehat{0}$ and a maximal element $\widehat{1}$. For any pair $z \leq x$ in $B$, we can consider the interval $[z, x]=\{y \in B \mid z \leq y \leq x\}$. Assume that $B$ is graded in the sense that for every $x \in B$, every maximal chain in the interval $[\widehat{0}, x]$ has the same length $\rho(x)$. We call $\rho: B \rightarrow \mathbb{N}$ the rank function of $B$, and call $\rho(\widehat{1})$ the rank of $B$. Then $B$ is Eulerian if every interval $[z, x]$ with $z<x$ has as many elements of odd rank as even rank.

Example 4.1 The poset of faces of a polytope $P$ (including the empty face) is an Eulerian poset under inclusion, with $\rho(Q)=\operatorname{dim} Q+1$, for any face $Q$ of $P$. Throughout this paper, the empty polytope has dimension -1 . 
Example 4.2 If $B$ is a poset, then $B^{*}$ is the poset with the same elements as $B$ and all orderings reversed. In particular, $B$ is Eulerian if and only if $B^{*}$ is Eulerian.

The $g$-polynomial of an Eulerian poset is defined recursively and was introduced by Stanley [51, Corollary 6.7].

Definition 4.3 Let $B$ be an Eulerian poset of $\operatorname{rank} n$. If $n=0$, then $g(B ; t)=1$. If $n>0$, then $g(B ; t)$ is the unique polynomial of degree strictly less the $n / 2$ satisfying

$$
t^{n} g\left(B ; t^{-1}\right)=\sum_{x \in B}(t-1)^{n-\rho(x)} g([\widehat{0}, x] ; t) .
$$

Example 4.4 By considering the leading terms above, we see that $g(B ; t)$ has constant term 1 , and linear term $\#\{x \in B \mid \rho(x)=1\}-n$.

Example 4.5 One can verify that $g(B ; t)=1$ if and only if $B$ is the Boolean algebra on $r$ elements for some $r$ [6, Remark 4.2].

We will need the following theorem of Stanley.

Theorem 4.6 [51, Corollary 8.3] If B is Eulerian and has positive rank, then

$$
\sum_{x \in B}(-1)^{\rho(x)} g([\widehat{0}, x] ; t) g\left([x, \widehat{1}]^{*} ; t\right)=\sum_{x \in B}(-1)^{\rho(x)} g\left([\widehat{0}, x]^{*} ; t\right) g([x, \widehat{1}] ; t)=0 .
$$

\subsection{Polyhedral subdivisions}

A polyhedral subdivision of a polytope $P \subseteq \mathbb{R}^{n}$ is a subdivision of $P$ into a finite number of polytopes such that the intersection of any two polytopes is a (possibly empty) face of both. A polyhedral subdivision is a rational (respectively, lattice) subdivision if each polytope has rational (respectively, integral) vertices. A natural class of lattice polyhedral subdivisions are the regular lattice subdivisions. They are induced by a function $\omega: S \rightarrow \mathbb{Z}$, called an integral height function, for some finite subset $S \subseteq P \cap \mathbb{Z}^{n}$ containing the vertices of $P$. The cells of the subdivision are the projections of the bounded faces of the convex hull UH of $\{(u, \lambda) \mid u \in S, \lambda \geq \omega(u)\} \subseteq \mathbb{R}^{n} \times \mathbb{R}$. The bounded faces of UH form the graph of a function $v: P \rightarrow \mathbb{R}$, called the convex graph of $\omega$. Note that $\left.v\right|_{S}$ is not necessarily the same as $\left.\omega\right|_{S}$. Let $\mathcal{S}(\nu)$ denote the regular lattice polyhedral subdivision induced by $\omega$. Then $v$ is convex and piecewise $\mathbb{Q}$-affine with respect to $\mathcal{S}(v)$, and takes integer values on the vertices of $\mathcal{S}(v)$. Conversely, if $v: P \rightarrow \mathbb{R}$ is a convex function that is piecewise $\mathbb{Q}$-affine with respect to a lattice polyhedral subdivision $\mathcal{S}$, such that $v$ takes integer values on the vertices of $\mathcal{S}$, then the restriction of $v$ to the vertices of $\mathcal{S}$ is an integral height function such that $v$ is the corresponding convex graph and $\mathcal{S}$ is a refinement of $\mathcal{S}(v)$. For more details, including the general definition of a regular polyhedral subdivision, see $[31,36]$.

By abuse of notation, we write $\mathcal{S}$ for the poset of faces (including the empty face) of a polyhedral subdivision $\mathcal{S}$ of $P$. Let $[\emptyset, P]$ denote the face poset of $P$. In what follows, we will consider the function

$$
\sigma: \mathcal{S} \rightarrow[\emptyset, P]
$$

where $\sigma(\emptyset)=\emptyset$, and for a nonempty cell $F \in \mathcal{S}, \sigma(F)$ is the smallest face of $P$ containing $F$. As a poset, the link $\operatorname{lk}_{\mathcal{S}}(F)$ of $F$ in $\mathcal{S}$ consists of all cells $F^{\prime}$ of $\mathcal{S}$ that contain $F$.

We will need the following two closely related polynomials. In the case $F=\emptyset$, the local $h$-polynomial defined below was introduced by Stanley in [51, Corollary 7.7]. In the case 
of a triangulation of a simplex, it was introduced by Athanasiadis [1,2], and the general case of a polyhedral subdivision of a polytope was first considered by Nill and Schepers [41], where it was called the 'relative local $h$-polynomial'. A more general definition of the local $h$-polynomial appears in [29, Definition 4.1].

Definition 4.7 Let $\mathcal{S}$ be a polyhedral subdivision of a polytope $P$. If $F$ is a (possibly empty) cell of $\mathcal{S}$, then the $h$-polynomial of $\operatorname{lk}_{\mathcal{S}}(F)$ is defined by

$$
t^{\operatorname{dim} P-\operatorname{dim} F} h\left(\mathrm{lk}_{\mathcal{S}}(F) ; t^{-1}\right)=\sum_{\substack{F^{\prime} \in \mathcal{S} \\ F \subseteq F^{\prime}}} g\left(\left[F, F^{\prime}\right] ; t\right)(t-1)^{\operatorname{dim} P-\operatorname{dim} F^{\prime}} .
$$

The local $h$-polynomial of $F$ in $\mathcal{S}$ is the polynomial

$$
l_{P}(\mathcal{S}, F ; t)=\sum_{\sigma(F) \subseteq Q \subseteq P}(-1)^{\operatorname{dim} P-\operatorname{dim} Q} h\left(\operatorname{lk}_{\left.\mathcal{S}\right|_{Q}}(F) ; t\right) g\left([Q, P]^{*} ; t\right) .
$$

When $F=\emptyset$, we write $h(\mathcal{S} ; t)=h\left(\operatorname{lk}_{\mathcal{S}}(F) ; t\right)$ and $l_{P}(\mathcal{S} ; t)=l_{P}(\mathcal{S}, F ; t)$.

Example 4.8 If $\mathcal{S}$ is the trivial subdivision of $P$, with cells of $\mathcal{S}$ given by the faces of $P$, then one can verify that $h\left(\operatorname{lk}_{\mathcal{S}}(Q) ; t\right)=g([Q, P] ; t)$ and $l_{P}(\mathcal{S}, Q ; t)=0$ whenever $Q$ is a (possibly empty) proper face of $P$.

We state some properties of these polynomials below. The nonnegativity of the $g$ polynomial and $h$-polynomial and the local $h$-polynomial when $F=\emptyset$ is due to Stanley [51, Theorem 5.2, Theorem 7.9].

Remark 4.9 [29, Theorem 6.1] Let $\mathcal{S}$ be a rational polyhedral subdivision of a lattice polytope $P$. For any cell $F \in \mathcal{S}$ and face $Q$ of $P$, the polynomials $g\left([Q, P]^{*} ; t\right), g([Q, P] ; t)$, $h\left(\operatorname{lk}_{\mathcal{S}}(F) ; t\right)$ and $l_{P}(\mathcal{S}, F ; t)$ have nonnegative coefficients. We have the symmetry

$$
l_{P}(\mathcal{S}, F ; t)=t^{\operatorname{dim} P-\operatorname{dim} F} l_{P}\left(\mathcal{S}, F ; t^{-1}\right) .
$$

If $\mathcal{S}$ is a regular subdivision, then the coefficients of $l_{P}(\mathcal{S}, F ; t)$ are symmetric and unimodal.

In order that one may compute examples, we list some of the coefficients below (see [29, Example 3.13,Remark 3.32,Example 4.9,Example 4.10]).

Example 4.10 Let $\mathcal{S}$ be a polyhedral subdivision of a polytope $P$. Let $F$ be a cell of $\mathcal{S}$. Let

$$
\begin{aligned}
& \lambda=\#\left\{F \subseteq F^{\prime} \mid \operatorname{dim} F^{\prime}=\operatorname{dim} F+1\right\}, \\
& \lambda^{\prime}=\#\left\{F \subseteq F^{\prime} \mid \operatorname{dim} F^{\prime}=\operatorname{dim} F+1, \sigma\left(F^{\prime}\right)=P\right\} .
\end{aligned}
$$

The constant term of $h\left(\operatorname{lk}_{\mathcal{S}}(F) ; t\right)$ is 1 , and the linear coefficient is $\lambda-(\operatorname{dim} P-\operatorname{dim} F)$. If $\sigma(F)=P$, then $h\left(\operatorname{lk}_{\mathcal{S}}(F) ; t\right)=l_{P}(\mathcal{S}, F ; t)$ is symmetric. Assume that $\sigma(F) \neq P$. Then $h\left(\operatorname{lk}_{\mathcal{S}}(F) ; t\right)$ has degree at most $\operatorname{dim} P-\operatorname{dim} F-1$, and the coefficient of $t^{\operatorname{dim} P-\operatorname{dim} F-1}$ is $\lambda^{\prime}$. Moreover, $l_{P}(\mathcal{S}, F ; t)$ is symmetric with constant term 0 and linear term

$$
\left\{\begin{array}{cl}
\lambda^{\prime}-1 & \text { if } \operatorname{dim} P-\operatorname{dim} \sigma(F)=1, \\
\lambda^{\prime} & \text { if } \operatorname{dim} P-\operatorname{dim} \sigma(F)>1
\end{array}\right.
$$

\subsection{Weighted Ehrhart theory}

Fix a lattice polytope $P$ with respect to a lattice $M$. After possibly replacing $M$ with a sublattice, we may assume that $\operatorname{dim} P=\operatorname{dim} M_{\mathbb{R}}$. Under an isomorphism $M \cong \mathbb{Z}^{n}$, the 
normalized volume $\operatorname{Vol}(P) \in \mathbb{Z}_{>0}$ of $P$ with respect to $M$ is $n$ ! times the Euclidean volume of $P$. Let $v: P \rightarrow \mathbb{R}$ be the convex graph of an integral height function on $P$, with corresponding regular lattice polyhedral subdivision $\mathcal{S}(\nu)$ of $P$.

Remark 4.11 The setup above is slightly different than the 'weight 0' setup in [52], although the same arguments hold. We explain the relationship below. In [52], we consider a stacky fan $\left(N, \Sigma,\left\{b_{i}\right\}\right)$, where $\Sigma$ is a full-dimensional simplicial fan with respect to a lattice $N$, and $\left\{b_{i}\right\}$ is a choice of a nonzero lattice point on each ray of $\Sigma$. We let $\psi$ be the piecewise $\mathbb{Q}$-linear function with respect to $\Sigma$ satisfying $\psi\left(b_{i}\right)=1$, and let $Q=\left\{v \in N_{\mathbb{R}} \mid \psi(v) \leq 1\right\}$. If $Q$ is a convex polytope, then we may set $M=N, P=Q$ and $v=\psi$ above to recover this setup. Here $\Sigma$ induces a triangulation $\mathcal{S}$ of $P$ refining $\mathcal{S}(v)$ such that $v$ takes integer values on the vertices of $\mathcal{S}$. We also note that the definition of the weighted $h^{*}$-polynomial below is slightly different (although equivalent) to that in [52] (see Example 5.13).

Let $C$ be the cone over $P \times\{1\}$ in $M_{\mathbb{R}} \times \mathbb{R}$, and extend $v$ by linearity to a piecewise $\mathbb{Q}$-linear function on $C$. For a nonnegative integer $m$, we will often identify $m P$ with $C \cap\left(M_{\mathbb{R}} \times\{m\}\right)$ and $M_{\mathbb{R}}$ with $M_{\mathbb{R}} \times\{m\}$. We define a weight function

$$
\begin{aligned}
& w: C \cap(M \times \mathbb{Z}) \rightarrow \mathbb{Q} / \mathbb{Z}, \\
& w(v)=[v(v)] .
\end{aligned}
$$

Note that adding a global affine function with respect to $M$ to $v$ does not change $w$. Recall from Remark 2.3 that the group algebra $\mathbb{Z}[\mathbb{Q} / \mathbb{Z}]$ admits a conjugation action such that $\overline{[k]}=[-k]$, and a 'forgetful' $\mathbb{Z}$-algebra homomorphism $\mathbb{Z}[\mathbb{Q} / \mathbb{Z}] \rightarrow \mathbb{Z},[k] \mapsto 1$. The weighted Ehrhart polynomial is defined by

$$
f(P, v ; m)=\sum_{v \in m P \cap M} w(v) \in \mathbb{Z}[\mathbb{Q} / \mathbb{Z}]
$$

for every nonnegative integer $m$. As in Remark 2.4, the corresponding polynomial with coefficients in $\mathbb{Z}$ is the usual Ehrhart polynomial of $P[20,21]$, defined by $f(P ; m)=$ $\#(m P \cap M)$ for every nonnegative integer $m$. By [52, Theorem 3.7], $f(P, v ; m)$ is a polynomial in $m$ of $\operatorname{degree} \operatorname{dim} P$, and for every positive integer $m$,

$$
(-1)^{\operatorname{dim} P} f(P, v ;-m)=\sum_{v \in \operatorname{Int}(m P) \cap M} \overline{w(v)} \in \mathbb{Z}[\mathbb{Q} / \mathbb{Z}] .
$$

The latter result is known as weighted Ehrhart reciprocity, since it reduces to the classical Ehrhart reciprocity: $f(P ;-m)=\#(\operatorname{Int}(m P) \cap M)$ for every positive integer $m$ [22]. In fact, it is shown in [52, Remark 3.10] that weighted Ehrhart reciprocity generalizes Ehrhart reciprocity for rational polytopes. Let $\mathcal{S}$ be a lattice polyhedral decomposition refining $\mathcal{S}(v)$ such that $v$ takes integer values on the vertices of $\mathcal{S}$. For each maximal cell $F \in \mathcal{S}$, let $m_{F}$ be the minimal positive integer such that $\left.m_{F} v\right|_{F}$ is an affine function with respect to $M$. Then one verifies that the leading coefficient of $f(P, v ; m)$ as a polynomial in $m$ is given by:

$$
\frac{1}{(\operatorname{dim} P) !} \sum_{\substack{F \in \mathcal{S} \\ \operatorname{dim} F=\operatorname{dim} P}} \frac{\operatorname{Vol}(F)}{m_{F}} \sum_{i=0}^{m_{F}-1}\left[i / m_{F}\right] \in \mathbb{Z}[\mathbb{Q} / \mathbb{Z}]
$$


where $\operatorname{Vol}(F)$ is the normalized volume of $F$ and $\operatorname{Vol}(F) / m_{F}$ is a positive integer. Define the weighted $h^{*}$-polynomial $h^{*}(P, v ; u) \in \mathbb{Z}[\mathbb{Q} / \mathbb{Z}][u]$ by

$$
\sum_{m \geq 0} f(P, v ; m) u^{m}=\frac{h^{*}(P, v ; u)}{(1-u)^{\operatorname{dim} P+1}} .
$$

When $P$ is the empty polytope, we set $h^{*}(P, v ; u)=1$. As in Remark 2.4, the corresponding polynomial with coefficients in $\mathbb{Z}$ is the usual $h^{*}$-polynomial $h^{*}(P ; u) \in \mathbb{Z}[u]$ of $P$ introduced by Stanley in [50]. We define the local weighted $h^{*}$-polynomial $l^{*}(P, v ; u) \in \mathbb{Z}[\mathbb{Q} / \mathbb{Z}][u]$ by

$$
l^{*}(P, v ; u)=\sum_{Q \subseteq P}(-1)^{\operatorname{dim} P-\operatorname{dim} Q} h^{*}\left(Q,\left.v\right|_{Q} ; u\right) g\left([Q, P]^{*} ; u\right) .
$$

Here the sum runs over all faces of $P$ including the empty face. When $P$ is the empty polytope, we set $l^{*}(P, v ; u)=1$. As in Remark 2.4 , the corresponding polynomial with coefficients in $\mathbb{Z}$ is the usual local $h^{*}$-polynomial $l^{*}(P ; u) \in \mathbb{Z}[u]$ of $P$, introduced by Stanley in [51, Example 7.13] and later independently by Borisov and Mavlyutov in [5]. By Theorem 4.6 and Remark 4.9, the weighted $h^{*}$-polynomial can be recovered as a 'nonnegative' combination of local weighted $h^{*}$-polynomials:

$$
h^{*}(P, v ; u)=\sum_{Q \subseteq P} l^{*}\left(Q,\left.v\right|_{Q} ; u\right) g([Q, P] ; u) .
$$

We present some properties and examples below. For the corresponding invariants over $\mathbb{Z}$, proofs of the properties below can be found in [6] and [29].

Example 4.12 One may verify that (6) translates into the following:

$$
h^{*}(P, v ; 1)=\sum_{\substack{F \in \mathcal{S} \\ \operatorname{dim} F=\operatorname{dim} P}} \frac{\operatorname{Vol}(F)}{m_{F}} \sum_{i=0}^{m_{F}-1}\left[i / m_{F}\right] \in \mathbb{Z}[\mathbb{Q} / \mathbb{Z}],
$$

where $m_{F}$ is the minimal positive integer such that $\left.m_{F} v\right|_{F}$ is an affine function with respect to $M$.

Example 4.13 Suppose that $P$ is a simplex and $v$ is $\mathbb{Q}$-affine, and let $v_{0}, \ldots, v_{n}$ denote the primitive integer vectors on the rays of $C$ corresponding to the vertices of $P$. Let Box $=\left\{v \in C \cap(M \times \mathbb{Z}) \mid v=\sum_{i=0}^{n} a_{i} v_{i}, 0 \leq a_{i}<1\right\}$. If pr $: N_{\mathbb{R}} \times \mathbb{R} \rightarrow \mathbb{R}$ denotes projection onto the second coordinate, then

$$
\begin{aligned}
h^{*}(P, v ; u) & =\sum_{v \in \operatorname{Box}} w(v) u^{\operatorname{pr}(v)} \in \mathbb{Z}[\mathbb{Q} / \mathbb{Z}][u], \\
l^{*}(P, v ; u) & =\sum_{v \in \operatorname{Box} \cap \operatorname{Int}(C)} w(v) u^{\operatorname{pr}(v)} \in \mathbb{Z}[\mathbb{Q} / \mathbb{Z}][u] .
\end{aligned}
$$

Remark 4.14 One may verify that weighted Ehrhart reciprocity is equivalent to the following statements about the weighted $h^{*}$-polynomial and local weighted $h^{*}$-polynomial, respectively (cf. [29, Lemma 7.11]). Firstly, the weighted $h^{*}$-polynomial is acceptable in the sense of Stanley [51]. That is,

$$
u^{\operatorname{dim} P+1} \overline{h^{*}\left(P, v ; u^{-1}\right)}=\sum_{Q \subseteq P} h^{*}\left(Q,\left.v\right|_{Q} ; u\right)(u-1)^{\operatorname{dim} P-\operatorname{dim} Q},
$$


where the above sum ranges over all (possibly empty) faces $Q$ of $P$. Secondly, the local weighted $h^{*}$-polynomial is symmetric in the sense that

$$
l^{*}(P, v ; u)=u^{\operatorname{dim} P+1} \overline{l *\left(P, v ; u^{-1}\right)} .
$$

Example 4.15 If $P$ is nonempty, then $h^{*}(P, v ; u)$ and $l^{*}(P, v ; u)$ have degree in $u$ at most $\operatorname{dim} P$, with the coefficient of $u^{\operatorname{dim} P}$ in both cases equal to $\sum_{v \in \operatorname{Int}(P) \cap M} \overline{w(v)}$. By the symmetry in Remark 4.14 above, this implies that $l^{*}(P, v ; u)$ has constant term 0 and linear coefficient $\sum_{v \in \operatorname{Int}(P) \cap M} w(v)$. The polynomial $h^{*}(P, v ; u)$ has constant term 1 and linear coefficient $\sum_{v \in P \cap M} w(v)-\operatorname{dim} P-1$.

Remark 4.16 Let $\mathcal{S}$ be a lattice polyhedral subdivision of $P$ that refines $\mathcal{S}(v)$ such that $v$ takes integer values on the vertices of $\mathcal{S}$. Then it follows from the definitions that for every positive integer $m$,

$$
f(P, v ; m)=\sum_{F \in \mathcal{S}} \sum_{v \in \operatorname{Int}(m F) \cap M} w(v) .
$$

Using weighted Ehrhart reciprocity and following the proof of [29, Lemma 7.12], the above statement is equivalent to any of the three statements below:

$$
\begin{aligned}
h^{*}(P, v ; u) & =\sum_{\substack{F \in \mathcal{S} \\
\sigma(F)=P}} h^{*}\left(F,\left.v\right|_{F} ; u\right)(u-1)^{\operatorname{dim} P-\operatorname{dim} F,} \\
h^{*}(P, v ; u) & =\sum_{F \in \mathcal{S}} l^{*}\left(F,\left.v\right|_{F} ; u\right) h\left(\mathrm{lk}_{\mathcal{S}}(F) ; u\right), \\
l^{*}(P, v ; u) & =\sum_{F \in \mathcal{S}} l^{*}\left(F,\left.v\right|_{F} ; u\right) l_{P}(\mathcal{S}, F ; u) .
\end{aligned}
$$

Remark 4.17 One may always choose a lattice triangulation $\mathcal{S}$ of $P$ that refines $\mathcal{S}(v)$ such that $v$ takes integer values on the vertices of $\mathcal{S}$. In fact, there exists a choice with the same vertices as $\mathcal{S}(v)$ (see, for example, [42]). Then Remark 4.16 together with Example 4.13 gives explicit formulas that one may use to compute $h^{*}(P, v ; u)$ and $l^{*}(P, v ; u)$ in practice. In particular, using Remark 4.9, it follows that all integer coefficients in $h^{*}(P, v ; u)$ and $l^{*}(P, v ; u)$ are nonnegative.

Remark 4.18 The following lower bound theorem generalizes [29, Theorem 7.20] in the case when $v \equiv 0$ : if we write $l^{*}(P, v ; u)=\sum_{k \in \mathbb{Q} / \mathbb{Z}} l^{*}(P, v ; u)_{[k]}[k]$ and $l^{*}(P, v ; u)_{[0]}=$ $l_{1}^{*}(P, v)_{[0]} u+\cdots+l_{\operatorname{dim} P}^{*}(P, v)_{[0]} u^{\operatorname{dim} P}$, then

$$
l_{1}^{*}(P, v)_{[0]}=\#\left\{v \in \operatorname{Int}(P) \cap \mathbb{Z}^{d} \mid w(v)=0\right\} \leq l_{i}^{*}(P, v)_{[0]}
$$

for $1 \leq i \leq \operatorname{dim} P$. This follows by the proof of [29, Theorem 7.20]. Explicitly, consider a regular, lattice polyhedral subdivision $\mathcal{S}$ of $P$ that refines $\mathcal{S}(v)$ such that the lattice points of weight 0 in $P$ are precisely the vertices of $\mathcal{S}$. Then $v$ takes integer values on the vertices of $\mathcal{S}$, and every positive-dimensional cell in $\mathcal{S}$ contains no interior lattice points of weight 0 . By Remark 4.16 and Example 4.15,

$$
l^{*}(P, v ; u)_{[0]}=l_{P}(\mathcal{S} ; u)+\beta(u) u^{2},
$$

where $l_{P}(\mathcal{S} ; u)$ has nonnegative, symmetric, unimodal coefficients and the polynomial $\beta(u)$ has nonnegative integer coefficients. The result follows. 
Example 4.19 The following example appeared in [52, Example 1.1]. Let $M=\mathbb{Z}^{2}$ and let $P$ be the lattice polytope with vertices $(1,0),(0,2),(-1,2),(-2,1),(-2,0)$ and $(0,-1)$. Let $v: P \rightarrow \mathbb{R}$ be the piecewise $\mathbb{Q}$-linear function satisfying $v(0)=0$ and $v(v)=1$ for every vertex $v$ of $P$. We write $f(P, v ; m)=\sum_{[k] \in \mathbb{Q} / \mathbb{Z}} f_{[k]}(P, v ; m)[k]$ below. Then the invariants above may be computed to be:

$$
\begin{aligned}
& h^{*}(P, v ; u)=1+4 u+u^{2}+2 u(1+u)[1 / 2]+u[2 / 3]+u^{2}[1 / 3], \\
& h^{*}(P ; u)=1+7 u+4 u^{2}, \\
& l^{*}(P, v ; u)=u(1+u)(1+2[1 / 2])+u[2 / 3]+u^{2}[1 / 3], \\
& l^{*}(P ; u)=4 u(1+u) .
\end{aligned}
$$

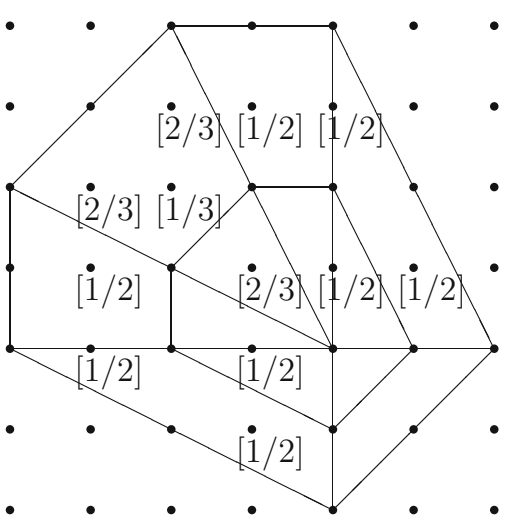

\subsection{Mixed invariants}

We introduce our main combinatorial invariants below. As in Remark 2.4, the corresponding invariants with coefficients in $\mathbb{Z}$ first appeared in [29]. A geometric description of these invariants is provided in Corollary 5.12.

Definition 4.20 Let $P$ be a lattice polytope and let $v: P \rightarrow \mathbb{R}$ be the convex graph of an integral height function on $P$, with corresponding regular lattice polyhedral subdivision $\mathcal{S}(v)$ of $P$. Then the weighted limit mixed $h^{*}$-polynomial $h^{*}(P, v ; u, v) \in \mathbb{Z}[\mathbb{Q} / \mathbb{Z}][u, v]$ of $(P, v)$ is

$$
h^{*}(P, v ; u, v):=\sum_{F \in \mathcal{S}(v)} v^{\operatorname{dim} F+1} l^{*}\left(F,\left.v\right|_{F} ; u v^{-1}\right) h\left(\mathrm{lk}_{\mathcal{S}(v)}(F) ; u v\right) .
$$

The local weighted limit mixed $h^{*}$-polynomial $l^{*}(P, v ; u, v) \in \mathbb{Z}[\mathbb{Q} / \mathbb{Z}][u, v]$ of $(P, v)$ is

$$
l^{*}(P, v ; u, v):=\sum_{F \in \mathcal{S}(v)} v^{\operatorname{dim} F+1} l^{*}\left(F,\left.v\right|_{F} ; u v^{-1}\right) l_{P}(\mathcal{S}(v), F ; u v) .
$$

The weighted refined limit mixed $h^{*}$-polynomial $h^{*}(P, v ; u, v, w) \in \mathbb{Z}[\mathbb{Q} / \mathbb{Z}][u, v, w]$ of $(P, v)$ is

$$
h^{*}(P, v ; u, v, w)=\sum_{Q \subseteq P} w^{\operatorname{dim} Q+1} l^{*}\left(Q,\left.v\right|_{Q} ; u, v\right) g\left([Q, P] ; u v w^{2}\right) .
$$

If $P$ is empty, then $h^{*}(P, v ; u, v, w)=h^{*}(P, v ; u, v)=l^{*}(P, v ; u, v)=1$. We write

$$
h^{*}(P, v ; u, v, w)=1+u v w^{2} \cdot \sum_{0 \leq p, q, r \leq \operatorname{dim} P-1} h_{p, q, r}^{*}(P, v) u^{p} v^{q} w^{r},
$$

where $h_{p, q, r}^{*}(P, v)=\sum_{[k] \in \mathbb{Q} / \mathbb{Z}} h_{p, q, r}^{*}(P, v)_{[k]}[k] \in \mathbb{Z}[\mathbb{Q} / \mathbb{Z}]$, for some $h_{p, q, r}^{*}(P, v)_{[k]} \in \mathbb{Z}$. 
We summarize the main properties of these invariants in the following theorem. They may all be deduced from the definitions and the properties in the previous sections, and hence we omit the proof. We refer the reader to [29, Theorem 9.2, Theorem 9.3,Theorem 9.4] for a similar statement for the corresponding invariants with coefficients in $\mathbb{Z}$.

Theorem 4.21 Let $P$ be a lattice polytope and let $v: P \rightarrow \mathbb{R}$ be the convex graph of an integral height function on $P$, with corresponding regular lattice polyhedral subdivision $\mathcal{S}(v)$ of $P$. Then the following holds:

(1) The weighted refined limit mixed $h^{*}$-polynomial satisfies the following symmetries:

$$
\begin{aligned}
& h^{*}(P, v ; u, v, w)=\overline{h^{*}(P, v ; v, u, w)} \\
& h^{*}(P, v ; u, v, w)=\overline{h^{*}\left(P, v ; u^{-1}, v^{-1}, u v w\right)}
\end{aligned}
$$

(2) The weighted refined limit mixed $h^{*}$-polynomial specializes to the weighted limit mixed $h^{*}$-polynomial:

$$
h^{*}(P, v ; u, v, 1)=h^{*}(P, v ; u, v) .
$$

(3) The weighted refined limit mixed $h^{*}$-polynomial specializes to the weighted $h^{*}$ polynomial:

$$
h^{*}(P, v ; u, 1,1)=h^{*}(P, v ; u, 1)=h^{*}(P, v ; u) .
$$

(4) The local weighted limit mixed $h^{*}$-polynomial specializes to the local weighted $h^{*}$ polynomial:

$$
l^{*}(P, v ; u, 1)=l^{*}(P, v ; u) .
$$

(5) The degree of $h^{*}(P, v ; u, v, w)$ as a polynomial in $w$ is at most $\operatorname{dim} P+1$. Moreover, the coefficient of $w^{\operatorname{dim} P+1}$ is the local weighted limit mixed $h^{*}$-polynomial $l^{*}(P, v ; u, v)$. We have symmetries:

$$
l^{*}(P, v ; u, v)=\overline{l^{*}(P, v ; v, u)}=(u v)^{\operatorname{dim} P+1} \overline{l^{*}\left(P, v ; u^{-1}, v^{-1}\right)} .
$$

(6) We have the following:

$$
\begin{aligned}
h^{*}(P, v ; u, v, w) & =\sum_{Q \subseteq P} w^{\operatorname{dim} Q+1} l^{*}\left(Q,\left.v\right|_{Q} ; u, v\right) g\left([Q, P] ; u v w^{2}\right), \\
w^{\operatorname{dim} P+1} l^{*}(P, v ; u, v) & =\sum_{Q \subseteq P}(-1)^{\operatorname{dim} P-\operatorname{dim} Q} h^{*}\left(Q,\left.v\right|_{Q} ; u, v, w\right) g\left([Q, P]^{*} ; u v w^{2}\right) .
\end{aligned}
$$

(7) We have the following:

$$
h^{*}(P, v ; u, v)=\sum_{\substack{F \in \mathcal{S}(v) \\ \sigma(F)=P}} h^{*}\left(F,\left.v\right|_{F} ; u, v\right)(u v-1)^{\operatorname{dim} P-\operatorname{dim} F}
$$

(8) If $v$ is $\mathbb{Q}$-affine, then $l^{*}(P, v ; u, v)=v^{\operatorname{dim} P+1} l^{*}\left(P, v ; u v^{-1}\right)$ and

$$
h^{*}(P, v ; u, v, w)=h^{*}(P, v ; u w, v w) .
$$

(9) The coefficients $h_{p, q, r}^{*}(P, v)_{[k]}$ are nonnegative integers. For $0 \leq j \leq r$ and $[k] \in \mathbb{Q} / \mathbb{Z}$, the sequence $\left\{h^{i+j, i, r}(P, v)_{[k]} \mid 0 \leq i \leq r-j\right\}$ is symmetric and unimodal. Moreover, $h_{j, 0, r}^{*}(P, v)_{[0]} \leq h_{j-i, i, r}^{*}(P, v)_{[0]}$ for $0 \leq i \leq j$ and $h_{r, j, r}^{*}(P, v)_{[0]} \leq h_{r-i, j+i, r}^{*}(P, v)_{[0]}$ for $0 \leq i \leq r-j$. 
Remark 4.22 As in [29, Section 9], after fixing $r$, one may view the coefficients $h_{p, q, r}^{*}(P, v)$ in a diamond, for example, by placing $h_{p, q, r}^{*}(P, v)$ at point $(q-p, p+q)$ in $\mathbb{Z}^{2}$. The resulting diamond of coefficients is symmetric with respect to its symmetry about the horizontal axis and symmetric up to conjugation with respect to its symmetry about the vertical axis. Fixing $[k] \in \mathbb{Q} / \mathbb{Z}$, the coefficients of $[k]$ in each vertical strip of the diamond form a symmetric, unimodal sequence. The coefficients of [0] in each horizontal strip of the diamond satisfy the following lower bound theorem: the first entry is a lower bound for the other entries.

Example 4.23 As in Example 4.13, suppose that $P$ is a simplex and $v$ is $\mathbb{Q}$-affine. For every face $Q$ of $P$, let $C_{Q}$ denote the cone over $Q \times\{1\}$ in $M_{\mathbb{R}} \times \mathbb{R}$, and set $C=C_{P}$. Note that if $Q$ is empty, then $C_{Q}$ is the origin. Let $v_{0}, \ldots, v_{n}$ denote the primitive integer vectors on the rays of $C$ corresponding to the vertices of $P$. Let Box $=\{v \in C \cap(M \times \mathbb{Z}) \mid$ $\left.v=\sum_{i=0}^{n} a_{i} v_{i}, 0 \leq a_{i}<1\right\}$. If pr: $N_{\mathbb{R}} \times \mathbb{R} \rightarrow \mathbb{R}$ denotes projection onto the second coordinate, then

$$
\begin{aligned}
h^{*}(P, v ; u, v, w) & =\sum_{Q \subseteq P} \sum_{v \in \operatorname{Box} \cap \operatorname{Int}\left(C_{Q}\right)} w(v) u^{\operatorname{pr}(v)} v^{\operatorname{dim} Q+1-\operatorname{pr}(v)} w^{\operatorname{dim} Q+1} \in \mathbb{Z}[\mathbb{Q} / \mathbb{Z}][u, v, w] . \\
l^{*}(P, v ; u, v) & =\sum_{v \in \operatorname{Box} \cap \operatorname{Int}(C)} w(v) u^{\operatorname{pr}(v)} v^{\operatorname{dim} P+1-\operatorname{pr}(v)} \in \mathbb{Z}[\mathbb{Q} / \mathbb{Z}][u, v] .
\end{aligned}
$$

We present explicit descriptions of some of the coefficients below.

Example 4.24 Let $P$ be a lattice polytope and let $v: P \rightarrow \mathbb{R}$ be the convex graph of an integral height function on $P$, with corresponding regular lattice polyhedral subdivision $\mathcal{S}(v)$ of $P$. Then for $q, r>0$,

$$
\begin{aligned}
& h_{0, q, r}^{*}(P, v)=h_{r-q, r, r}^{*}(P, v)=\overline{h_{q, 0, r}^{*}(P, v)}=\overline{h_{r, r-q, r}^{*}(P, v)}=\sum_{\begin{array}{c}
F \in \mathcal{S}(v) \\
\operatorname{dim} F=q+1 \\
\operatorname{dim} \sigma(F)=r+1
\end{array}} \sum_{v \in \operatorname{Int}(F) \cap M} w(v), \\
& h_{0,0, r}^{*}(P, v)=h_{r, r, r}^{*}(P, v)=\sum_{\substack{F \in \mathcal{S}(v) \\
\operatorname{dim} F \leq 1 \\
\operatorname{dim} \sigma(F)=r+1}} \sum_{v \in \operatorname{Int}(F) \cap M} w(v), \\
& \operatorname{dim} P+1+h_{0,0,0}^{*}(P, v)=\sum_{\substack{Q \subseteq P \\
\operatorname{dim} Q \leq 1}} \sum_{v \in \operatorname{Int}(Q) \cap M} w(v) .
\end{aligned}
$$

When $\operatorname{dim} P=2$, this gives an explicit description of $h^{*}(P, v ; u, v, w)$ :

$$
\begin{aligned}
h^{*}(P ; v ; u, v, w)= & 1+u v w^{2}\left[h_{0,0,0}^{*}(P, v)+w\left[(1+u v) h_{0,0,1}^{*}(P, v)+v h_{0,1,1}^{*}(P, v)\right.\right. \\
& \left.\left.+u h_{0,1,1}^{*}(P, v)\right]\right] .
\end{aligned}
$$

When $\operatorname{dim} P=3$, this gives an explicit description of all terms in $h^{*}(P, v ; u, v, w)$ except $h_{1,1,2}^{*}$. The remaining term can be deduced from the expression for $h^{*}(P, v ; 1,1,1)=$ $h^{*}(P, v ; 1)$ in Example 4.12 . 
Example 4.25 In the case of Example 4.19, one computes:

$$
\begin{aligned}
h^{*}(P ; v ; u, v, w) & =1+u v w^{2}[3+w[(1+u v)(1+2[1 / 2])+v[2 / 3]+u[1 / 3]]], \\
h^{*}(P ; v ; u, v) & =1+u v[4+u v+2[1 / 2](1+u v)+v[2 / 3]+u[1 / 3]], \\
l^{*}(P ; v ; u, v) & =u v[(1+u v)(1+2[1 / 2])+v[2 / 3]+u[1 / 3]] .
\end{aligned}
$$

\section{Degenerations of hypersurfaces}

In this section, we use Theorem 3.2 together with the combinatorics of Sect. 4 to prove a formula for the equivariant refined limit Hodge-Deligne polynomial of a schön hypersurface of a torus. We also give an equivalent formula for the equivariant refined limit Hodge-Deligne polynomial associated with the intersection cohomology of a canonical compactification of such a hypersurface. The corresponding results for invariants with coefficients in $\mathbb{Z}$ appear in [30]. We will continue with the notation of Sects. 2 and 4.

Let $M$ be a lattice and let $N=\operatorname{Hom}(M, \mathbb{Z})$. Let $X^{\circ}=\{f=0\} \subseteq \operatorname{Spec} \mathbb{K}[M] \cong\left(\mathbb{K}^{*}\right)^{n}$ be a schön hypersurface, where $f=\sum_{u \in M} \gamma_{u}(t) x^{u}$ for some $\gamma_{u}(t) \in \mathbb{C}(t)$. The Newton polytope $P$ of $X^{\circ}$ is the convex hull of $S=\left\{u \in M \mid \gamma_{u}(t) \neq 0\right\}$ in $M_{\mathbb{R}}$. Note that $P$ may be viewed as a full-dimensional lattice polytope in its affine span with induced lattice structure, and $X^{\circ} \cong X^{\prime} \times\left(\mathbb{K}^{*}\right)^{k}$, for some $k$, where $X^{\prime}$ is a schön hypersurface in a torus of dimension $\operatorname{dim} P$ with Newton polytope $P$. Hence we may and will assume that $\operatorname{dim} P=n$.

Consider the induced integral height function $\omega_{f}: S \rightarrow \mathbb{Z}$ defined by $\omega_{f}(u)=\operatorname{ord}_{t} \gamma_{u}(t)$. Recall from Sect. 4.2 that we may consider the convex graph $v_{f}$ associated with $\omega_{f}$, with corresponding regular lattice polyhedral subdivision $\mathcal{S}\left(v_{f}\right)$.

Remark 5.1 Conversely, assume that $v: P \rightarrow \mathbb{R}$ is the convex graph of an integral height function on $P$. Since schönness is a generic condition, it follows that there exists a schön hypersurface $X^{\circ}=\{f=0\} \subseteq \operatorname{Spec} \mathbb{K}[M]$ such that $v_{f}=v[30$, Remark 5.1].

Tropical geometry of hypersurfaces reduces to the study of Newton polytopes and polyhedral subdivisions $[31,46]$. In particular, $\operatorname{Trop}\left(X^{\circ}\right)$ admits a polyhedral structure $\Sigma$ with cells $\gamma$ in inclusion-reversing correspondence with positive-dimensional cells $F$ of $\mathcal{S}\left(v_{f}\right)$, such that the bounded cells of $\Sigma$ correspond to the cells of $\mathcal{S}\left(v_{f}\right)$ not contained in the boundary of $P$. For example, if $F$ is a maximal dimensional cell in $\mathcal{S}\left(v_{f}\right)$, then $\left.v_{f}\right|_{F}$ is a $\mathbb{Q}$-affine function and the corresponding $\mathbb{Q}$-linear function is an rational point in $N_{\mathbb{Q}}$, also denoted $\left.v_{f}\right|_{F}$. Then $\gamma=-\left.v_{f}\right|_{F}$ is a vertex in $\Sigma$.

Example 5.2 Following on with Example 3.1 and Example 3.4, let $X^{\circ}=\left\{\sum_{i=1}^{n} x_{i}^{m_{i}}=\right.$ $\left.t^{e}\right\} \subseteq\left(\mathbb{K}^{*}\right)^{n}$, for some $m_{i} \in \mathbb{Z}_{>0}$ and $e \in \mathbb{Z}$. Let $f_{1}, \ldots, f_{n}$ denote the standard basis vectors of $M_{R} \cong \mathbb{R}^{n}$. Then $P$ is the convex hull of the origin and $\left\{m_{i} f_{i} \mid 1 \leq i \leq n\right\}$, and $v_{f}$ is the $\mathbb{Q}$-affine function corresponding to the $\mathbb{Q}$-linear function $\left(-e / m_{1}, \ldots,-e / m_{n}\right) \in N_{\mathbb{Q}}$. As we have seen, $\operatorname{Trop}\left(X^{\circ}\right)$ has the structure of a fan with vertex $\left(e / m_{1}, \ldots, e / m_{n}\right) \in N_{\mathbb{Q}}$.

Write $f=\sum_{u \in M} \lambda_{u} t^{\omega_{f}(u)} g_{u}(t) x^{u}$ for some $\lambda_{u} \in \mathbb{C}$ and $g_{u}(t) \in \mathbb{C}(t)$ such that $g(1)=1$. Let $F$ be a face of $\mathcal{S}\left(v_{f}\right)$ corresponding to a cell $\gamma$ in $\mathcal{S}\left(v_{f}\right)$. Then

$$
\operatorname{in}_{\gamma} X^{\circ}=\left\{\sum_{\substack{u \in F \cap M \\ \omega_{f}(u)=v_{f}(u)}} \lambda_{u} x^{u}=0\right\} \subseteq\left(\mathbb{C}^{*}\right)^{n} .
$$


Let $M_{F}$ denote the intersection of $M$ with the translation of the affine span of $F$ to the origin. Then equation in the right-hand side of (7) determines a schön complex hypersurface $V_{F}^{\circ} \in \operatorname{Spec} \mathbb{C}\left[M_{F}\right]$, and $\left.v_{f}\right|_{F}$ determines a $\mathbb{Q}$-linear function on $\left(M_{F}\right)_{\mathbb{Q}}$. Multiplication by $\exp \left(-\left.2 \pi \sqrt{-1} v_{f}\right|_{F}\right)$ determines a good $\widehat{\mu}$-action on $V_{F}^{\circ}$. In this case, Theorem 3.2 translates into the following corollary.

Corollary 5.3 Let $X^{\circ} \subseteq\left(\mathbb{K}^{*}\right)^{n}$ be a schön hypersurface, with associated Newton polytope and convex graph of an integral height function $(P, v)$ and $\operatorname{dim} P=n$. Then the motivic nearby fiber of $X^{\circ}$ is given by

$$
\psi_{X^{\circ}}=\sum_{\substack{F \in \mathcal{S}(v) \\ \sigma(F)=P}}\left[V_{F}^{\circ} \circlearrowleft \widehat{\mu}\right](1-\mathbb{L})^{\operatorname{dim} P-\operatorname{dim} F} .
$$

Here $\sigma(F)$ denotes the smallest face of $P$ containing $F$, and $V_{F}^{\circ}$ is a schön complex hypersurface of a torus with good $\widehat{\mu}$-action induced by multiplication by $\exp \left(-\left.2 \pi \sqrt{-1} \nu\right|_{F}\right)$.

Remark 5.4 In fact, the sum in Corollary 5.3 runs only over the positive-dimensional faces in $\mathcal{S}(v)$, since when $F$ is a vertex of $\mathcal{S}(v), V_{F}^{\circ}=\emptyset$. Here we follow the convention that $[V]=0 \in K_{0}^{\widehat{\mu}}\left(\operatorname{Var}_{\mathbb{C}}\right)$ if $V$ is empty.

Example 5.5 Let $X^{\circ} \subseteq\left(\mathbb{K}^{*}\right)^{n}$ be a schön hypersurface, with associated Newton polytope and convex graph of an integral height function $(P, v)$ and $\operatorname{dim} P=n$. Assume that $v$ is $\mathbb{Q}$-affine. Then the associated polyhedral subdivision $\mathcal{S}(v)$ of $P$ is trivial and Corollary 5.3 states that $\psi_{X^{\circ}}=\left[V_{P}^{\circ} \circlearrowleft \widehat{\mu}\right]$.

Example 5.6 Let $V^{\circ}=\left\{\sum_{u \in P \cap M} \lambda_{u} x^{u}=0\right\} \subseteq\left(\mathbb{C}^{*}\right)^{n}$ be a schön complex hypersurface with Newton polytope $P$ and $\operatorname{dim} P=n$. Let $v$ be the convex graph of an integral height function on $P$ and assume that $v$ is $\mathbb{Q}$-affine and $v(u) \in \mathbb{Z}$ whenever $\lambda_{u} \neq 0$. Then $V^{\circ}$ is invariant under multiplication by the associated element $\exp (-2 \pi \sqrt{-1} v) \in\left(\mathbb{C}^{*}\right)^{n}$, and we have an induced good $\widehat{\mu}$-action on $V^{\circ}$. For example, the hypersurfaces $V_{F}^{\circ}$ with corresponding good $\widehat{\mu}$-action in the statement of Corollary 5.3 all appear in this way.

In this case, the variety $X^{\circ}=\left\{f=\sum_{u \in P \cap M} \lambda_{u} t^{\nu(u)} x^{u}=0\right\} \subseteq\left(\mathbb{K}^{*}\right)^{n}$ is schön and may be viewed as a family $f: X^{\circ} \rightarrow \mathbb{C}^{*}$ with isomorphic fibers. Explicitly, we may identify $f^{-1}(1)$ with $V^{\circ}$, and multiplication by $\exp (-2 \pi \sqrt{-1} \lambda \nu) \in\left(\mathbb{C}^{*}\right)^{n}$, for any $\lambda \in \mathbb{C}$, gives an isomorphism between $f^{-1}(1)$ and $f^{-1}(\exp (2 \pi \sqrt{-1} \lambda))$. Considering $0 \leq \lambda \leq 1$, we see that we may identify the good $\widehat{\mu}$-action on $V^{\circ}$ above with the good $\widehat{\mu}$-action on $f^{-1}(1)$ induced by monodromy. Note that since the monodromy action is finite, the corresponding monodromy operator $T$ on cohomology is semi-simple. As in Example 5.5, Corollary 5.3 states that $\psi_{X^{\circ}}=\left[V^{\circ} \circlearrowleft \widehat{\mu}\right]$.

Recall the definition of the weighted refined limit mixed $h^{*}$-polynomial $h^{*}(P, v ; u, v, w) \in$ $\mathbb{Z}[\mathbb{Q} / \mathbb{Z}][u, v, w]$ from Definition 4.20. Our goal is to prove the following formula for the equivariant refined limit Hodge-Deligne polynomial.

Theorem 5.7 Let $X^{\circ} \subseteq\left(\mathbb{K}^{*}\right)^{n}$ be a schön hypersurface, with associated Newton polytope and convex graph of an integral height function $(P, v)$ and $\operatorname{dim} P=n$. Then the equivariant refined limit Hodge-Deligne polynomial $E\left(X_{\infty}^{\circ}, \widehat{\mu} ; u, v, w\right) \in \mathbb{Z}[\mathbb{Q} / \mathbb{Z}][u, v, w]$ of $X^{\circ}$ is given by 


$$
u v w^{2} E\left(X_{\infty}^{\circ}, \widehat{\mu} ; u, v, w\right)=\left(u v w^{2}-1\right)^{\operatorname{dim} P}+(-1)^{\operatorname{dim} P+1} h^{*}(P, v ; u, v, w) .
$$

We will also prove an equivalent statement involving intersection cohomology. We will use middle perversity throughout. As explained in detail in [30, Section 6], given a projective variety $X$ over $\mathbb{K}$, for each of the geometric invariants described in Section 2.3, one may consider the corresponding invariant with respect to intersection cohomology rather than cohomology, e.g., the equivariant refined limit Hodge-Deligne polynomial $E_{\text {int }}\left(X_{\infty}, \widehat{\mu} ; u, v, w\right)$ associated with the intersection cohomology of $X$. The key benefit of using intersection cohomology is the fact that the intersection cohomology groups of a complex projective variety admit a pure (rather than just mixed) Hodge structure.

We will need the following sum-over-strata formula. Let $X^{\circ} \subseteq\left(\mathbb{K}^{*}\right)^{n}$ be a schön hypersurface, with associated Newton polytope and convex graph of an integral height function $(P, v)$ and $\operatorname{dim} P=n$. Let $\Delta_{P}$ denote the normal fan to $P$ with cones $\sigma$ in inclusionreserving bijection with the faces $Q_{\sigma}$ of $P$. Let $X$ denote the closure of $X^{\circ}$ in the projective toric variety $\mathbb{P}\left(\Delta_{P}\right)_{\mathbb{K}}$ over $\mathbb{K}$ corresponding to $\Delta_{P}$. Then $X$ admits a stratification $X=\bigcup_{\sigma \in \Delta_{P}} X_{\sigma}^{\circ}$, where $X_{\sigma}^{\circ}$ is a schön hypersurface with Newton polytope $Q_{\sigma}$ and convex graph of an integral height function $\left.v\right|_{Q_{\sigma}}$. The theorem below is proved in the nonequivariant setting in [30, Theorem 6.1], but the proof goes through unchanged in our situation.

Theorem 5.8 [30, Theorem 6.1] Let $X^{\circ} \subseteq\left(\mathbb{K}^{*}\right)^{n}$ be a schön hypersurface, with associated Newton polytope and convex graph of an integral height function $(P, v)$ and $\operatorname{dim} P=n$. Let $X$ denote the closure of $X^{\circ}$ in the projective toric variety over $\mathbb{K}$ corresponding to the normal fan of $P$. Then the equivariant refined limit Hodge-Deligne polynomial obeys

$$
E_{\text {int }}\left(X_{\infty}, \widehat{\mu} ; u, v, w\right)=\sum_{\sigma \in \Delta_{P}} E\left(\left(X_{\sigma}^{\circ}\right)_{\infty}, \widehat{\mu} ; u, v, w\right) g\left([0, \sigma] ; u v w^{2}\right) .
$$

Our goal is to prove the following theorem.

Theorem 5.9 Let $X^{\circ} \subseteq\left(\mathbb{K}^{*}\right)^{n}$ be a schön hypersurface, with associated Newton polytope and convex graph of an integral height function $(P, v)$ and $\operatorname{dim} P=n$. Let $X$ denote the closure of $X^{\circ}$ in the projective toric variety over $\mathbb{K}$ corresponding to the normal fan of $P$. Then the equivariant refined limit Hodge-Deligne polynomial $E_{\mathrm{int}}\left(X_{\infty}, \widehat{\mu} ; u, v, w\right) \in$ $\mathbb{Z}[\mathbb{Q} / \mathbb{Z}][u, v, w]$ associated with the intersection cohomology of $X$ is given by

$$
u v w^{2} E_{\text {int }}\left(X_{\infty}, \widehat{\mu} ; u, v, w\right)=u v w^{2} E_{\text {int,Lef }}\left(P ; u v w^{2}\right)+(-1)^{\operatorname{dim} P+1} w^{\operatorname{dim} P+1} l^{*}(P, v ; u, v),
$$

where

$$
(t-1) E_{\text {int,Lef }}(P ; t)=t^{\operatorname{dim} P} g\left([\emptyset, P]^{*} ; t^{-1}\right)-g\left([\emptyset, P]^{*} ; t\right)
$$

is defined in terms of Stanley's g-polynomial (see Definition 4.3).

The following lemma is a formal consequence of Theorem 5.8. Details are provided in [30, Lemma 6.2].

Lemma 5.10 Theorem 5.7 and Theorem 5.9 are equivalent.

It is well known that all interesting cohomology above occurs in middle dimension. We review the relevant facts below and refer the reader to [30, Sections 5.3,6] for details. We 
have weak Lefschetz theorems stating that the Gysin maps $H_{c}^{m}\left(X_{\text {gen }}^{\circ}\right) \rightarrow H_{c}^{m+2}\left(\left(\mathbb{C}^{*}\right)^{n}\right)$ and $I H^{m}\left(X_{\text {gen }}\right) \rightarrow I H^{m+2}\left(\mathbb{P}\left(\Delta_{P}\right)_{\mathbb{C}}\right)$ are surjective for $m \geq n-1$ and an isomorphism if $m>n-1$. One may deduce from Theorem 5.8 that the monodromy maps associated with the cohomology with compact supports and intersection cohomology of $\left(\mathbb{K}^{*}\right)^{n}$ and $\mathbb{P}\left(\Delta_{P}\right)_{\mathbb{K}}$, respectively, are trivial. Since $X_{\text {gen }}^{\circ}$ is affine, $H_{c}^{m}\left(X_{\text {gen }}^{\circ}\right)=0$ for $m<n-1$. Also, Poincaré duality for intersection cohomology means that the Gysin isomorphism above determines $I H^{m}\left(X_{\text {gen }}\right)$ for $m<n-1$. We let $H_{\text {prim, } c}^{n-1}\left(X_{\infty}^{\circ}\right)$ and $I H_{\text {prim }}^{n-1}\left(X_{\infty}\right)$ denote the kernels of the above Gysin maps with induced filtrations. These groups constitute the 'interesting' cohomology. Consider the contributions of these groups (up to a sign) to the equivariant refined limit Hodge-Deligne polynomial:

$$
\begin{aligned}
E_{\text {prim }}\left(X_{\infty}^{\circ}, \widehat{\mu} ; u, v, w\right) & :=\sum_{p, q, r} \sum_{\alpha \in \mathbb{Q} / \mathbb{Z}} h^{p, q, r}\left(H_{\text {prim }, c}^{n-1}\left(X_{\infty}^{\circ}\right)\right)_{\alpha} \alpha u^{p} v^{q} w^{r}, \\
E_{\text {int,prim }}\left(X_{\infty}, \widehat{\mu} ; u, v, w\right) & :=\sum_{p, q, r} \sum_{\alpha \in \mathbb{Q} / \mathbb{Z}} h^{p, q, r}\left(I H_{\text {prim }}^{n-1}\left(X_{\infty}\right)\right)_{\alpha} \alpha u^{p} v^{q} w^{r} .
\end{aligned}
$$

Since primitive intersection cohomology is concentrated in $W$-degree equal to $\operatorname{dim} P-1$,

$$
E_{\text {int,prim }}\left(X_{\infty}, \widehat{\mu} ; u, v, w\right)=w^{\operatorname{dim} P-1} E_{\text {int,prim }}\left(X_{\infty}, \widehat{\mu} ; u, v, 1\right) .
$$

One may then verify (see [30, p.35]) that

$$
\begin{aligned}
u v w^{2} E\left(X_{\infty}^{\circ}, \widehat{\mu} ; u, v, w\right) & =\left(u v w^{2}-1\right)^{\operatorname{dim} P}+(-1)^{\operatorname{dim} P+1}\left(1+u v w^{2} E_{\text {prim }}\left(X_{\infty}^{\circ}, \widehat{\mu} ; u, v, w\right)\right), \\
E_{\text {int }}\left(X_{\infty}, \widehat{\mu} ; u, v, w\right) & =E_{\text {int,Lef }}\left(P ; u v w^{2}\right)+(-1)^{\operatorname{dim} P+1} E_{\text {int,prim }}\left(X_{\infty}, \widehat{\mu} ; u, v, w\right),
\end{aligned}
$$

where $E_{\text {int,Lef }}(P ; t)$ is defined in (8). We conclude that Theorem 5.9 is equivalent to the following:

$$
u v E_{\text {int,prim }}\left(X_{\infty}, \widehat{\mu} ; u, v, 1\right)=l^{*}(P, v ; u, v) .
$$

By Lemma 5.10, it follows that Theorem 5.7 is equivalent to its specialization at $w=1$ :

$$
u v E\left(X_{\infty}^{\circ}, \widehat{\mu} ; u, v\right)=(u v-1)^{\operatorname{dim} P}+(-1)^{\operatorname{dim} P+1} h^{*}(P, v ; u, v) .
$$

Before proceeding to the proof of Theorem 5.7 and Theorem 5.9, we will need the following theorem. In fact, it is a consequence of the proof that the theorem below is a special case of Theorem 5.7 and Theorem 5.9. An algorithm to compute the equivariant Hodge-Deligne polynomial below is given in [39, Section 2], extending an algorithm of Danilov and Khovanskii in the nonequivariant setting [14]. The theorem below generalizes a formula of Borisov and Mavlyutov in the nonequivariant setting [5].

Theorem 5.11 Let $V^{\circ}=\left\{\sum_{u \in P \cap M} \lambda_{u} x^{u}=0\right\} \subseteq\left(\mathbb{C}^{*}\right)^{n}$ be a schön complex hypersurface with Newton polytope $P$ and $\operatorname{dim} P=n$. Let $v$ be the convex graph of an integral height function on $P$, and assume that $v$ is $\mathbb{Q}$-affine and $v(u) \in \mathbb{Z}$ whenever $\lambda_{u} \neq 0$. Then $V^{\circ}$ is invariant under multiplication by the associated element $\exp (-2 \pi \sqrt{-1} v) \in\left(\mathbb{C}^{*}\right)^{n}$, and we have an induced good $\widehat{\mu}$-action on $V^{\circ}$. The corresponding equivariant Hodge-Deligne polynomial $E_{\widehat{\mu}}\left(V^{\circ} ; u, v\right) \in \mathbb{Z}[\mathbb{Q} / \mathbb{Z}][u, v]$ is given by:

$$
u v E_{\widehat{\mu}}\left(V^{\circ} ; u, v\right)=(u v-1)^{\operatorname{dim} P}+(-1)^{\operatorname{dim} P+1} h^{*}(P, v ; u, v) .
$$


Proof Recall from Example 5.6 that the variety $X^{\circ}=\left\{f=\sum_{u \in P \cap M} \lambda_{u} t^{\nu(u)} x^{u}=0\right\} \subseteq$ $\left(\mathbb{K}^{*}\right)^{n}$ is schön, and satisfies $\psi_{X^{\circ}}=\left[V^{\circ} \circlearrowleft \widehat{\mu}\right]$, and hence $E\left(X_{\infty}^{\circ}, \widehat{\mu} ; u, v\right)=E_{\widehat{\mu}}\left(V^{\circ} ; u, v\right)$. Moreover, the action of monodromy on cohomology is semi-simple and hence

$$
E\left(X_{\infty}^{\circ}, \widehat{\mu} ; u, v, w\right)=E\left(X_{\infty}^{\circ}, \widehat{\mu} ; u w, v w\right)=E_{\widehat{\mu}}\left(V^{\circ} ; u w, v w\right) .
$$

If $X=\cup_{Q \subseteq P} X_{Q}^{\circ}$ denotes the stratification in the statement of Theorem 5.8 , then $X_{Q}^{\circ}$ is defined by an equation of the form $\left\{\sum_{u \in Q \cap M} \lambda_{u} t^{\nu(u)} x^{u}=0\right\}$. It follows that if we restrict Theorem 5.7 to all $X^{\circ}$ that appear from complex schön hypersurfaces $V^{\circ}$ as above, then Theorem 5.7, Theorem 5.9 and (9) and (10) are all still equivalent when restricted to this class of varieties over $\mathbb{K}$.

With the notation above, [39, Theorem 2.7] states that

$$
u E_{\widehat{\mu}}\left(V^{\circ} ; u, 1\right)=(u-1)^{\operatorname{dim} P}+(-1)^{\operatorname{dim} P+1} h^{*}(P, v ; u) .
$$

This establishes (9) and (10) and hence Theorems 5.7 and 5.9 for $X^{\circ}$ under the specialization $v=1$. We conclude that

$$
\begin{aligned}
u w E_{\widehat{\mu}}\left(V^{\circ} ; u, w\right) & =E\left(X_{\infty}^{\circ}, \widehat{\mu} ; u w^{-1}, 1, w\right) \\
& =(u w-1)^{\operatorname{dim} P}+(-1)^{\operatorname{dim} P+1} h^{*}\left(P, v ; u w^{-1}, 1, w\right) .
\end{aligned}
$$

By (8) in Theorem 4.21, $h^{*}\left(P, v ; u w^{-1}, 1, w\right)=h^{*}(P, v ; u, w)$. This completes the proof.

We now complete the proofs of Theorems 5.7 and 5.9, which we have established are both equivalent to equivalent statements (9) and (10).

Proof By Corollary 5.3,

$$
E\left(X_{\infty}^{\circ}, \widehat{\mu} ; u, v\right)=\sum_{\substack{F \in \mathcal{S}(v) \\ \sigma(F)=P}} E_{\widehat{\mu}}\left(V_{F}^{\circ} ; u, v\right)(1-u v)^{\operatorname{dim} P-\operatorname{dim} F} .
$$

Substituting in Theorem 5.11 yields:

$$
\begin{aligned}
u v E\left(X_{\infty}^{\circ}, \widehat{\mu} ; u, v\right)= & \sum_{\substack{F \in \mathcal{S}(v) \\
\sigma(F)=P}}\left[(u v-1)^{\operatorname{dim} F}+(-1)^{\operatorname{dim} F+1} h^{*}\left(F,\left.v\right|_{F} ; u, v\right)\right] \\
& \times(1-u v)^{\operatorname{dim} P-\operatorname{dim} F} \\
= & (u v-1)^{\operatorname{dim} P}+(-1)^{\operatorname{dim} P+1} \\
& \times \sum_{\substack{F \in \mathcal{S}(v) \\
\sigma(F)=P}} h^{*}\left(F,\left.v\right|_{F} ; u, v\right)(u v-1)^{\operatorname{dim} P-\operatorname{dim} F} \\
= & (u v-1)^{\operatorname{dim} P}+(-1)^{\operatorname{dim} P+1} h^{*}(P, v ; u, v) .
\end{aligned}
$$

Here the last equality follows from (7) in Theorem 4.21. This establishes (10) as desired.

Using Remark 5.1 and the above proof, we immediately deduce the following geometric description of the combinatorial invariants introduced in Sect. 4. As in Remark 2.4, the corresponding result for invariants with coefficients in $\mathbb{Z}$ can be found in [30, Corollary 5.11,Corollary 6.3].

Corollary 5.12 Let $P$ be a full-dimensional lattice polytope in a lattice $M$ and let $v: P \rightarrow$ $\mathbb{R}$ be the convex graph of an integral height function on $P$. Let $X^{\circ}=\{f=0\} \subseteq$ Spec $\mathbb{K}[M]$ 
be a schön hypersurface with Newton polytope P such that $v_{f}=v$. Let $X$ denote the closure of $X^{\circ}$ in the projective toric variety over $\mathbb{K}$ corresponding to the normal fan of $P$. Then

$$
h^{*}(P, v ; u, v, w)=1+u v w^{2} \sum_{p, q, r} \sum_{\alpha \in \mathbb{Q} / \mathbb{Z}} h^{p, q, r}\left(H_{\mathrm{prim}, c}^{\operatorname{dim} P-1}\left(X_{\infty}^{\circ}\right)\right)_{\alpha} \alpha u^{p} v^{q} w^{r} .
$$

In particular, this specializes to:

$$
\begin{aligned}
h^{*}(P, v ; u, v) & =1+u v \sum_{p, q} \sum_{\alpha \in \mathbb{Q} / \mathbb{Z}} h^{p, q}\left(H_{\text {prim }, c}^{\operatorname{dim} P-1}\left(X_{\infty}^{\circ}\right)\right)_{\alpha} \alpha u^{p} v^{q}, \\
h^{*}(P, v ; u) & =1+u \sum_{p} \sum_{\alpha \in \mathbb{Q} / \mathbb{Z}} \operatorname{dim} \operatorname{Gr}_{F}^{p}\left(H_{\text {prim }, c}^{\operatorname{dim} P-1}\left(X_{\infty}^{\circ}\right)\right)_{\alpha} \alpha u^{p}, \\
h^{*}(P, v ; 1) & =1+\sum_{\alpha \in \mathbb{Q} / \mathbb{Z}} \operatorname{dim} H_{\text {prim }, c}^{\operatorname{dim} P-1}\left(X_{\infty}^{\circ}\right)_{\alpha}[\alpha] .
\end{aligned}
$$

Moreover,

$$
l^{*}(P, v ; u, v)=u v \sum_{p, q} \sum_{\alpha \in \mathbb{Q} / \mathbb{Z}} h^{p, q}\left(I H_{\mathrm{prim}}^{\operatorname{dim} P-1}\left(X_{\infty}\right)\right)_{\alpha} \alpha u^{p} v^{q} .
$$

This specializes to:

$$
\begin{aligned}
& l^{*}(P, v ; u)=u \sum_{p} \sum_{\alpha \in \mathbb{Q} / \mathbb{Z}} \operatorname{dim} \operatorname{Gr}_{F}^{p}\left(I H_{\mathrm{prim}}^{\operatorname{dim} P-1}\left(X_{\infty}\right)\right)_{\alpha} \alpha u^{p}, \\
& l^{*}(P, v ; 1)=\sum_{\alpha \in \mathbb{Q} / \mathbb{Z}} \operatorname{dim} I H_{\text {prim }}^{\operatorname{dim} P-1}\left(X_{\infty}\right)_{\alpha}[\alpha] .
\end{aligned}
$$

Example 5.13 Let $P$ be a lattice polytope in a lattice $N$ such that $P$ contains the origin in its relative interior, and let $\left\{b_{i}\right\}$ denote the vertices of $P$. Let $v: P \rightarrow \mathbb{R}$ be the piecewise $\mathbb{Q}$ affine function with value 0 at the origin and value 1 on the boundary of $P$. For an explicit example, see Example 4.19. Let $X^{\circ}=\{f=0\} \subseteq$ Spec $\mathbb{K}[N]$ be a schön hypersurface with Newton polytope $P$ such that $v_{f}=v$. For example, if $g$ is a schön complex polynomial with Newton polytope $P$, then take $f=\operatorname{tg}-\lambda$ for a generic choice of $\lambda \in \mathbb{C}^{*}$. Define the primitive spectrum of $f$ to be:

$$
\operatorname{sp}_{f}^{\text {prim }}(t):=1+\sum_{\beta \in(0,1] \cap \mathbb{Q}} \sum_{p, q} h^{p, q}\left(H_{\mathrm{prim}, c}^{n-1}\left(X_{\infty}^{\circ}\right)\right)_{\exp (2 \pi \sqrt{-1} \beta)} t^{p+\beta} .
$$

Consider the weighted $h^{*}$-polynomial $h^{*}(P, v ; u)=1+u \sum_{\beta \in(0,1]} \sum_{p} h_{p, \beta}^{*}[\beta] u^{p} \in$ $\mathbb{Z}[\mathbb{Q} / \mathbb{Z}][u]$, for some nonnegative integers $h_{p, \beta}^{*}$. We consider an alternative way of encoding this polynomial. Specifically, for some sufficiently divisible positive integer $N$, define

$$
\tilde{h}(P ; t):=1+\sum_{\beta \in(0,1]} \sum_{p} h_{p, \beta}^{*} t^{p+\beta} \in \mathbb{Z}\left[t^{1 / N}\right] .
$$

This was the definition of the weighted $h^{*}$-polynomial given in [52]. Note that the $h^{*}$ polynomial $h^{*}(P ; t)$ is obtained from $\tilde{h}(P ; t)$ by rounding up the exponents of $t$ to the nearest integer. By [52, Corollary 2.12], we have the symmetry:

$$
\widetilde{h}(P ; t)=t^{\operatorname{dim} P} \widetilde{h}\left(P ; t^{-1}\right) .
$$

Consider the complete fan $\Sigma^{\prime}$ in $N_{\mathbb{R}}$ with cones given by the cones over the proper faces of $P$, and let $\Sigma$ be a simplicial fan refinement of $\Sigma^{\prime}$ with the same rays. Then the vertices $\left\{b_{i}\right\}$ of $P$ constitute a choice of a nonzero lattice point on each ray of $\Sigma$. As in Remark 4.11, 
the triple $\Sigma=\left(N, \Sigma,\left\{b_{i}\right\}\right)$ is a stacky fan and we may consider the corresponding DeligneMumford stack $\mathcal{X}=\mathcal{X}(\Sigma)$ with coarse moduli space the toric variety associated with the fan $\Sigma$ [4]. The theory of orbifold cohomology, developed by Chen and Ruan [11,12], associates to $\mathcal{X}$ a finite-dimensional $\mathbb{Q}$-algebra $H_{\text {orb }}^{*}(\mathcal{X}, \mathbb{Q})$, graded by $\mathbb{Q}$.

By [52, Theorem 4.3], together with Corollary 5.12, we see that the primitive spectrum of $f$ coincides with the Betti polynomial of $\mathcal{X}$ :

$$
\operatorname{sp}_{f}^{\text {prim }}(t)=\sum_{i \in \mathbb{Q}} \operatorname{dim} H_{\mathrm{orb}}^{2 i}(\mathcal{X}, \mathbb{Q}) t^{i}=\widetilde{h}(P ; t) .
$$

For an explicit example (cf. [52, Example 1.1]), in Example 4.19 we computed:

$$
h^{*}(P, v ; u)=1+4 u+u^{2}+2 u(1+u)[1 / 2]+u[2 / 3]+u^{2}[1 / 3] .
$$

Equivalently,

$$
\widetilde{h}(P ; t)=1+2 t^{1 / 2}+t^{2 / 3}+4 t+t^{4 / 3}+2 t^{3 / 2}+t^{2} .
$$

\section{Applications to the monodromy of complex polynomials}

In this section, we apply Theorem 5.7 to deduce explicit combinatorial equations for some important invariants associated with the monodromy of complex polynomials. Recall that we identify the group $\mathbb{Q} / \mathbb{Z}$ with the group $\mathbb{S}_{\mathbb{Q}}^{1}$ of rational points on the circle $\{z \in \mathbb{C} \mid$ $|z|=1\}$, sending $[k] \in \mathbb{Q} / \mathbb{Z}$ to $\alpha=e^{2 \pi \sqrt{-1} k} \in \mathbb{S}_{\mathbb{Q}}^{1}$. We will continue with the notation of Sects. 2, 4 and 5 , and set $M=\mathbb{Z}^{n}$.

\subsection{Degenerations of hypersurfaces in affine space}

We first consider applications of the results of Sect. 5 for families of hypersurfaces of affine space. Let $X^{\circ} \subseteq\left(\mathbb{K}^{*}\right)^{n}$ be a schön hypersurface, with associated Newton polytope and convex graph of an integral height function $(P, v)$ and $\operatorname{dim} P=n$. Assume that $P \subseteq \mathbb{R}_{\geq 0}^{n}$, and for each (possibly empty) subset $S$ of $\{1, \ldots, n\}$, let $\mathbb{R}^{S}=\left\{\left(v_{1}, \ldots, v_{n}\right) \mid v_{i}=0\right.$ if $\left.i \notin S\right\}$, $\mathbb{Z}^{S}=\mathbb{R}^{S} \cap \mathbb{Z}^{n}$ and $P^{S}=P \cap \mathbb{R}^{S}$. We assume that $P$ is convenient in the sense that $\operatorname{dim} P^{S}$ equals the cardinality $|S|$ of $S$ for every subset $S$ of $\{1, \ldots, n\}$. Equivalently, $P$ contains the origin and has nonzero intersection with each ray through a coordinate vector. We call a face (including the empty face) of $P$ that does not contain the origin, a face at infinity, and write $P_{\infty}$ for the union of faces at infinity of $P$. Then the faces of $P$ are precisely the faces at infinity together with $\left\{P^{S} \mid S \subseteq\{1, \ldots, n\}\right\}$. Let $X$ denote the closure of $X^{\circ}$ in $\mathbb{K}^{n}$. Then $X_{\text {gen }} \subseteq \mathbb{C}^{n}$ is a smooth hypersurface, and as in Sect. 5, one has a weak Lefschetz result (see, for example, [14, Corollary 3.8]) stating that $H_{c}^{2(n-1)}\left(X_{\text {gen }}\right)=\mathbb{C}$ with trivial monodromy action and the only other nonzero cohomology is in middle dimension $H_{c}^{n-1}\left(X_{\text {gen }}\right)$. By Corollary 5.3, the motivic nearby fiber of $X$ is given by

$$
\psi_{X}=\sum_{S \subseteq\{1, \ldots, n\}} \sum_{\substack{F \in \mathcal{S}(v) \\ \sigma(F)=P^{S}}}\left[V_{F}^{\circ} \circlearrowleft \widehat{\mu}\right](1-\mathbb{L})^{|S|-\operatorname{dim} F},
$$

where $\sigma(F)$ is the smallest face of $P$ containing $F$. After rearranging terms, Theorem 5.7 implies that

$$
u v w^{2} E\left(X_{\infty}, \widehat{\mu} ; u, v, w\right)=\left(u v w^{2}\right)^{n}+(-1)^{n-1} \sum_{S \subseteq\{1, \ldots, n\}}(-1)^{n-|S|} h^{*}\left(P^{S},\left.v\right|_{P} ; u, v, w\right) .
$$


Specializing by setting $w=1$ gives a formula for the equivariant limit Hodge-Deligne polynomial. We may specialize further by setting $v=1$ and then $u=1$. In particular, by Example 4.12, we have the following formula for the eigenvalues (with multiplicity) of the action of monodromy on the cohomology of $X_{\text {gen }}$ :

$$
\sum_{\alpha \in \mathbb{Q} / \mathbb{Z}} \operatorname{dim} H_{c}^{n-1}\left(X_{\infty}\right)_{\alpha} \alpha=\sum_{S \subseteq\{1, \ldots, n\}} \sum_{\substack{\left.F \in \mathcal{S}(v)\right|_{p S} \\ \operatorname{dim} F=\operatorname{dim} P^{S}}}(-1)^{n-|S|} \frac{\operatorname{Vol}(F)}{m_{F}} \sum_{i=0}^{m_{F}-1}\left[i / m_{F}\right]
$$

where $m_{F}$ is the minimal positive integer such that $\left.m_{F} v\right|_{F}$ is an affine function with respect to $\mathbb{Z}^{S}$. Here $\operatorname{Vol}(F)$ is the normalized volume of $F$, and $\operatorname{Vol}(F) / m_{F}$ is a positive integer.

Remark 6.1 With the setup above, do not assume that $P$ is convenient, and instead only assume that $P$ contains the origin. Then we may not apply the same weak Lefschetz argument. However, we still obtain the following formulas for the motivic nearby fiber and equivariant refined limit Hodge-Deligne polynomial:

$$
\begin{aligned}
& \psi_{X}=\sum_{S \subseteq\{1, \ldots, n\}}(-1)^{|S|-\operatorname{dim} P^{S}} \sum_{\substack{F \in \mathcal{S}(\nu) \\
\sigma(F)=P^{S}}}\left[V_{F}^{\circ} \circlearrowleft \widehat{\mu}\right](1-\mathbb{L})^{|S|-\operatorname{dim} F} . \\
& u v w^{2} E\left(X_{\infty}, \widehat{\mu} ; u, v, w\right)=\left(u v w^{2}\right)^{n} \\
& \quad+\sum_{S \subseteq\{1, \ldots, n\}}(-1)^{\operatorname{dim} P^{S}-1}\left(u v w^{2}-1\right)^{|S|-\operatorname{dim} P^{S}} h^{*}\left(P^{S},\left.v\right|_{P^{S}} ; u, v, w\right) .
\end{aligned}
$$

Specializing at $u=v=w=1$ gives:

$$
\begin{aligned}
E\left(X_{\infty}, \widehat{\mu} ; 1,1,1\right) & =\sum_{m} \sum_{\alpha \in \mathbb{Q} / \mathbb{Z}}(-1)^{m} \operatorname{dim} H_{c}^{m}\left(X_{\infty}\right)_{\alpha} \alpha \\
& =\sum_{\substack{\emptyset \neq S \subseteq\{1, \ldots, n\} \\
\operatorname{dim} P^{S}=|S|}}(-1)^{|S|-1} \sum_{\begin{array}{c}
\left.F \in \mathcal{S}(v)\right|_{p} S \\
\operatorname{dim} F=\operatorname{dim} P^{S}
\end{array}} \frac{\operatorname{Vol}(F)}{m_{F}} \sum_{i=0}^{m_{F}-1}\left[i / m_{F}\right],
\end{aligned}
$$

where $m_{F}$ is the minimal positive integer such that $\left.m_{F} v\right|_{F}$ is an affine function with respect to $\mathbb{Z}^{S}$.

Assume further that the restriction of $v$ to $P_{\infty}$ is constant Substitute in Definition 4.20 and observe that $g\left(\left[P^{S}, P^{S^{\prime}}\right] ; t\right)=1$ for any $S \subseteq S^{\prime}$ by Example 4.5. By assumption, for every face $Q$ at infinity, $l^{*}\left(Q,\left.v\right|_{Q} ; u, v\right)=v^{\operatorname{dim} Q+1} l^{*}\left(Q ; u v^{-1}\right)$. In this case, we may rewrite (12) as:

$$
u v w^{2} E\left(X_{\infty}, \widehat{\mu} ; u, v, w\right)=\left(u v w^{2}\right)^{n}+(-1)^{n-1}\left[F(u w, v w)+w^{n+1} l^{*}(P, v ; u, v)\right],
$$

where

$$
\begin{aligned}
& F(u, v):=\sum_{Q \subseteq P_{\infty}} v^{\operatorname{dim} Q+1} l^{*}\left(Q ; u v^{-1}\right) G(Q ; u v), \\
& G(Q ; t):=\sum_{\substack{S \subseteq\{1, \ldots, n\} \\
Q \subseteq P^{S}}}(-1)^{n-|S|} g\left(\left[Q, P^{S}\right] ; t\right) .
\end{aligned}
$$


Using Theorem 4.6 and Example 4.8, we have:

$$
\begin{aligned}
G(Q ; t) & =\sum_{Q \subseteq Q^{\prime} \subseteq P_{\infty}}(-1)^{n-1-\operatorname{dim} Q^{\prime}} g\left(\left[Q, Q^{\prime}\right] ; t\right) g\left(\left[Q^{\prime}, P\right]^{*} ; t\right) \\
& =\left[\sum_{\substack{S \subseteq\{1, \ldots, n\} \\
Q \subseteq P^{S}}}(-1)^{n-|S|} h\left(\operatorname{lk}_{\left.\mathcal{S}(v)\right|_{P} S}(Q) ; t\right)\right]-l_{P}(\mathcal{S}(v), Q ; u v) .
\end{aligned}
$$

Example 6.2 With the notation above, let $Q$ be a face at infinity and consider $G(Q ; t)$. By definition, $G(Q ; t)$ has degree strictly less than $(\operatorname{dim} P-\operatorname{dim} Q) / 2$. If $\operatorname{Int}(Q) \subseteq \mathbb{R}_{>0}^{n}$, then $G(Q ; t)=g([Q, P] ; t)$ has constant term 1 . Otherwise, $G(Q ; t)$ has no constant term. Using Example 4.4, when $Q=\emptyset$, we compute that the linear coefficient of $G(Q ; t)$ is the number of vertices of $P$ contained in $\mathbb{R}_{>0}^{n}$.

One then computes that $F(u, v)$ has the form

$$
\begin{aligned}
F(u, v)= & {\left[\sum_{\substack{Q \subseteq P_{\infty} \\
\operatorname{dim} Q \leq 1 \\
\operatorname{Int}(Q) \subseteq \mathbb{R}_{>0}^{n}}} \#\left(\operatorname{Int}(Q) \cap \mathbb{Z}^{n}\right)\right] u v } \\
& +\left[\sum_{\substack{Q \subseteq P_{\infty} \\
\operatorname{dim} Q=2 \\
\operatorname{Int}(Q) \subseteq \mathbb{R}_{>0}^{n}}} \#\left(\operatorname{Int}(Q) \cap \mathbb{Z}^{n}\right)\right] u v(u+v)+\beta(u, v),
\end{aligned}
$$

where every term in $\beta(u, v)$ has combined degree in $u$ and $v$ at least 4.

Observe that every term in $F(u w, v w)$ has combined degree in $u$ and $v$ equal to its degree in $w$. Hence, the only contribution above corresponding to a nontrivial action of monodromy is $(-w)^{n+1} l^{*}(P, v ; u, v)$. We conclude that we have the following corollary.

Corollary 6.3 Let $X^{\circ} \subseteq\left(\mathbb{K}^{*}\right)^{n}$ be a schön hypersurface, with associated Newton polytope and convex graph of an integral height function $(P, v)$ and $\operatorname{dim} P=n$. Let $X \subseteq \mathbb{K}^{n}$ denote the closure of $X^{\circ}$ in $\mathbb{K}^{n}$. Assume that $P \subseteq \mathbb{R}_{\geq 0}^{n}$ is convenient, and the restriction of $v$ to every face at infinity is constant. Then the action of monodromy is trivial on the graded pieces $\mathrm{Gr}_{r}^{W} H_{c}^{n-1}\left(X_{\text {gen }}\right)$ of the Deligne weight filtration for $r \neq n-1$, and we have the following explicit combinatorial formula for the equivariant limit mixed Hodge numbers of $\mathrm{Gr}_{n-1}^{W} H_{c}^{n-1}\left(X_{\text {gen }}\right)$ :

$$
u v \sum_{p, q} \sum_{\alpha \in \mathbb{Q} / \mathbb{Z}} h^{p, q}\left(G r_{n-1}^{W} H_{c}^{n-1}\left(X_{\infty}\right)\right)_{\alpha} \alpha u^{p} v^{q}=l^{*}(P, v ; u, v) .
$$

In particular, one may use Definition 2.6 to give a formula for the Jordan block structure of the action of monodromy on $G r_{n-1}^{W} H_{c}^{n-1}\left(X_{\mathrm{gen}}\right)$.

Example 6.4 With the notation of Corollary 6.3, let $n=2$ and consider $X \subseteq \mathbb{K}^{2}$. Using Example 4.24 and Example 6.2, we compute

$$
E\left(X_{\infty}, \widehat{\mu} ; u, v, w\right)=u v w^{2}-\left[b+w\left[h_{0,0,1}^{*}(P, v)(1+u v)+h_{0,1,1}^{*}(P, v) v+\overline{h_{0,1,1}^{*}(P, v)} u\right]\right]
$$


where $b=\#\left(\partial P \cap \mathbb{Z}_{>0}^{2}\right), \partial P$ denotes the boundary of $P$, and

$$
h_{0,0,1}^{*}(P, v)=\sum_{\substack{F \in \mathcal{S}(v) \\ \operatorname{dim} F \leq 1 \\ \sigma(F)=P}} \sum_{v \in \operatorname{Int}(F) \cap \mathbb{Z}^{2}} w(v), \quad h_{0,1,1}^{*}(P, v)=\sum_{\substack{F \in \mathcal{S}(v) \\ \operatorname{dim} F=2 \\ \sigma(F)=P}} \sum_{v \in \operatorname{Int}(F) \cap \mathbb{Z}^{2}} w(v) .
$$

In particular, the action of monodromy on $G r_{0}^{W} H_{c}^{1}\left(X_{\text {gen }}\right)$ is trivial and $\operatorname{dim} G r_{0}^{W} H_{c}^{1}\left(X_{\text {gen }}\right)=$ $b$. The equivariant limit mixed Hodge numbers of $G r_{1}^{W} H_{c}^{1}\left(X_{\text {gen }}\right)$ are given by

$$
\begin{aligned}
\sum_{\alpha \in \mathbb{Q} / \mathbb{Z}} h^{0,0}\left(G r_{1}^{W} H_{c}^{1}\left(X_{\infty}\right)\right)_{\alpha} \alpha & =\sum_{\alpha \in \mathbb{Q} / \mathbb{Z}} h^{1,1}\left(G r_{1}^{W} H_{c}^{1}\left(X_{\infty}\right)\right)_{\alpha} \alpha=h_{0,0,1}^{*}(P, v), \\
\sum_{\alpha \in \mathbb{Q} / \mathbb{Z}} h^{0,1}\left(G r_{1}^{W} H_{c}^{1}\left(X_{\infty}\right)\right)_{\alpha} \alpha & =\sum_{\alpha \in \mathbb{Q} / \mathbb{Z}} h^{1,0}\left(G r_{1}^{W} H_{c}^{1}\left(X_{\infty}\right)\right)_{\alpha} \alpha^{-1}=h_{0,1,1}^{*}(P, v) .
\end{aligned}
$$

The Jordan normal form of the action of monodromy on $\mathrm{Gr}_{1}^{W} H_{c}^{1}\left(X_{\mathrm{gen}}\right)$ has

$$
h^{0,1}\left(G r_{1}^{W} H_{c}^{1}\left(X_{\infty}\right)\right)_{\alpha}+h^{1,0}\left(G r_{1}^{W} H_{c}^{1}\left(X_{\infty}\right)\right)_{\alpha}
$$

Jordan blocks of size 1 with eigenvalue $\alpha$, and $h^{0,0}\left(G r_{1}^{W} H_{c}^{1}\left(X_{\infty}\right)\right)_{\alpha}$ Jordan blocks of size 2 with eigenvalue $\alpha$.

Remark 6.5 Specializing by setting $w=1$ above gives a formula for the equivariant limit mixed Hodge numbers:

$$
u v \sum_{p, q} \sum_{\alpha \in \mathbb{Q} / \mathbb{Z}} h^{p, q}\left(H_{c}^{n-1}\left(X_{\infty}\right)\right)_{\alpha} \alpha u^{p} v^{q}=F(u, v)+l^{*}(P, v ; u, v) .
$$

Substituting in the definitions and simplifying, the right-hand side may be rewritten as follows:

$$
\begin{aligned}
& \sum_{F \in \mathcal{S}(v)} v^{\operatorname{dim} F+1} l^{*}\left(F,\left.v\right|_{F} ; u v^{-1}\right) l_{P}(\mathcal{S}(v), F ; u v) \\
& F \nsubseteq P_{\infty} \\
& +\sum_{Q \subseteq P_{\infty}} v^{\operatorname{dim} Q+1} l^{*}\left(Q ; u v^{-1}\right) \sum_{\substack{S \subseteq\{1, \ldots, n\} \\
Q \subseteq P^{S}}}(-1)^{n-|S|} h\left(\operatorname{lk}_{\left.\mathcal{S}(v)\right|_{P S}}(Q) ; u v\right)
\end{aligned}
$$

We present the following two examples of the above situation. Below we let $f \in$ $\mathbb{C}\left[x_{1}, \ldots, x_{n}\right]$ be a complex polynomial with Newton polytope $\mathrm{NP}(f)$. For every face $Q$ of $\mathrm{NP}(f)$, we let $\Delta_{Q}$ denote the convex hull of $Q$ and the origin. We set $P=\Delta_{\mathrm{NP}}(f)$.

We assume that $f$ is convenient in the sense that $\mathrm{NP}(f)$ has nonzero intersection with each ray through a coordinate vector [34]. Recall that for a complex hypersurface of a torus, schön is also called nondegenerate. Let $\Gamma_{+}(f)=\mathrm{NP}(f)+\mathbb{R}_{\geq 0}^{n}$ denote the Newton polyhedron of $f$. Observe that every bounded face of $\Gamma_{+}(f)$ is a face of $N P(f)$. We let $\Gamma_{f}$ denote the union of the bounded faces of $\Gamma_{+}(f)$.

Remark 6.6 For any proper face $Q$ of $\mathrm{NP}(f)$ not containing the origin, consider a $\mathbb{Q}$-affine function $v$ on $\Delta_{Q}$ with value $m \pm 1$ at the origin, and value $m$ on $Q$, for some integer $m$. Consider $l^{*}(Q, v ; u) \in \mathbb{Z}[\mathbb{Q} / \mathbb{Z}][u]$ as a sum of integer-valued polynomials indexed by $\alpha \in \mathbb{Q} / \mathbb{Z}$. Then the coefficient of $\alpha=1$ is zero. To see this, one may for example use Remark 4.16 to reduce to the case when $Q$ is a simplex, and then apply Example 4.13.

Example 6.7 (Monodromy at 0 ) Assume that $f \in \mathbb{C}\left[x_{1}, \ldots, x_{n}\right]$ has no constant term, $f$ is convenient and $\{f=0\} \subseteq\left(\mathbb{C}^{*}\right)^{n}$ defines a schön hypersurface. Then for a general 
choice of $\lambda \in \mathbb{C}^{*}, X^{\circ}=\{f=\lambda t\} \subseteq\left(\mathbb{K}^{*}\right)^{n}$ defines a schön, convenient hypersurface with Newton polytope $P$, and $v_{0}:=v$ is the piecewise $\mathbb{Q}$-affine function on $P$ with value 1 at the origin and value identically 0 on $N P(f)$. The cells of $\mathcal{S}\left(v_{0}\right)$ are given by the union of $\{Q \mid Q \subseteq \mathrm{NP}(f)\}$ and $\left\{\Delta_{Q} \mid Q \subseteq \Gamma_{f}\right\}$. By (11), we have the following equation for the motivic nearby fiber:

$$
\psi_{X}=\sum_{Q \subseteq \Gamma_{f}}\left[V_{\Delta_{Q}}^{\circ} \circlearrowleft \widehat{\mu}\right](1-\mathbb{L})^{\operatorname{dim} \sigma\left(\Delta_{Q}\right)-\operatorname{dim} \Delta_{Q}}+\sum_{\substack{Q \subseteq N P(f) \\ Q \nsubseteq P_{\infty}}}\left[V_{Q}^{\circ}\right](1-\mathbb{L})^{\operatorname{dim} \sigma(Q)-\operatorname{dim} Q}
$$

By Corollary 6.3, the action of monodromy is trivial on the graded pieces $G r_{r}^{W} H_{c}^{n-1}\left(X_{\text {gen }}\right)$ of the Deligne weight filtration for $r \neq n-1$, and the equivariant limit mixed Hodge numbers of $G r_{n-1}^{W} H_{c}^{n-1}\left(X_{\text {gen }}\right)$ are given by:

$$
u v \sum_{p, q} \sum_{\alpha \in \mathbb{Q} / \mathbb{Z}} h^{p, q}\left(G r_{n-1}^{W} H_{c}^{n-1}\left(X_{\infty}\right)\right)_{\alpha} \alpha u^{p} v^{q}=l^{*}\left(P, v_{0} ; u, v\right) .
$$

By Remarks 6.5 and 6.6, we have the following formulas for the equivariant limit mixed Hodge numbers of $H_{c}^{n-1}\left(X_{\text {gen }}\right)$ :

$$
\begin{aligned}
& u v \sum_{p, q} \sum_{\substack{\alpha \in \mathbb{Q} / \mathbb{Z} \\
\alpha \neq 1}} h^{p, q}\left(H_{c}^{n-1}\left(X_{\infty}\right)\right)_{\alpha} \alpha u^{p} v^{q} \\
& =\sum_{Q \subseteq \Gamma_{f}} v^{\operatorname{dim} \Delta_{Q}+1} l^{*}\left(\Delta_{Q},\left.v_{0}\right|_{\Delta_{Q}} ; u v^{-1}\right) l_{P}\left(\mathcal{S}\left(v_{0}\right), \Delta_{Q} ; u v\right), \\
& u v \sum_{p, q} h^{p, q}\left(H_{c}^{n-1}\left(X_{\infty}\right)\right){ }_{1} u^{p} v^{q} \\
& =\sum_{\substack{Q \subseteq \mathrm{NP}(f) \\
Q \nsubseteq P_{\infty}}} v^{\operatorname{dim} Q+1} l^{*}\left(Q,\left.v_{0}\right|_{Q} ; u v^{-1}\right) l_{P}\left(\mathcal{S}\left(v_{0}\right), Q ; u v\right), \\
& +\sum_{Q \subseteq P_{\infty}} v^{\operatorname{dim} Q+1} l^{*}\left(Q ; u v^{-1}\right) \\
& \times \sum_{\substack{S \subseteq\{1, \ldots, n\} \\
Q \subseteq P^{S}}}(-1)^{n-|S|} h\left(\mathrm{lk}_{\left.\mathcal{S}\left(v_{0}\right)\right|_{P S}}(Q) ; u v\right) .
\end{aligned}
$$

By (13), we have a formula for the eigenvalues (with multiplicity) of the action of monodromy on the cohomology of $X_{\text {gen }}$. Explicitly, $\sum_{\alpha \in \mathbb{Q} / \mathbb{Z}} \operatorname{dim} H_{c}^{n-1}\left(X_{\infty}\right)_{\alpha} \alpha$ is equal to

$$
\begin{gathered}
\sum_{\substack{Q \subseteq \Gamma_{f} \\
\operatorname{dim} \sigma(\Delta Q)=\operatorname{dim} \Delta_{Q}}}(-1)^{n-1-\operatorname{dim} Q} \operatorname{Vol}(Q) \sum_{i=0}^{m(Q)-1}[i / m(Q)] \\
\sum_{\substack{Q \subseteq N \mathrm{NP}(f) \\
Q \nsubseteq P_{\infty} \\
\operatorname{dim} \sigma(Q)=\operatorname{dim} Q}}(-1)^{n-\operatorname{dim} Q} \operatorname{Vol}(Q),
\end{gathered}
$$

where $\operatorname{Vol}(Q)$ is the normalized volume of $Q$ and $m(Q)$ is the lattice distance of $Q$ from the origin, i.e., $m(Q)$ is the minimal positive integer such that $\left.m(Q) v_{0}\right|_{\Delta_{Q}}$ is an affine function 
with respect to the lattice given by intersecting $M$ with the affine span of $\Delta_{Q}$. When $Q$ is empty, $\operatorname{Vol}(Q)=m(Q)=1$ and $\operatorname{dim} Q=-1$.

Example 6.8 (Monodromy at infinity) Assume that $f \in \mathbb{C}\left[x_{1}, \ldots, x_{n}\right]$ is convenient. Assume that $f$ is schön at infinity, meaning that $\left\{\left.f\right|_{Q}=0\right\} \subseteq\left(\mathbb{C}^{*}\right)^{n}$ defines a smooth hypersurface whenever $Q$ is a face of $P$ at infinity. Then for a general choice of $\lambda \in \mathbb{C}^{*}$, $X^{\circ}=\{f t=\lambda\} \subseteq\left(\mathbb{K}^{*}\right)^{n}$ defines a schön, convenient hypersurface with Newton polytope $P$, and $v_{\infty}:=v$ is the piecewise $\mathbb{Q}$-affine function on $P$ with value 0 at the origin and value identically 1 on the faces at infinity of $P$. The cells of $\mathcal{S}\left(v_{\infty}\right)$ are given by the union of $\left\{Q \mid Q \subseteq P_{\infty}\right\}$ and $\left\{\Delta_{Q} \mid Q \subseteq P_{\infty}\right\}$. By (11), we have the following equation for the motivic nearby fiber:

$$
\psi_{X}=\sum_{Q \subseteq P_{\infty}}\left[V_{\Delta_{Q}}^{\circ} \widehat{\mu}\right](1-\mathbb{L})^{\operatorname{dim} \sigma\left(\Delta_{Q}\right)-\operatorname{dim} \Delta_{Q}} .
$$

The motivic nearby fiber at infinity of a convenient polynomial $f$ was introduced independently and from different perspectives in [39] and [45], and an explicit formula is given in [39, Theorem 5.3], which agrees with our formula for the motivic nearby fiber above. In particular, the motivic nearby fiber at infinity is equal to the usual motivic nearby fiber of $X$.

Let $\Delta^{\infty}$ be the simplex $P \cap\left\{x_{1}+\cdots+x_{n}=\epsilon\right\}$ for fixed $\epsilon>0$ sufficiently small. Let $\mathcal{S}_{\infty}$ denote the regular, rational polyhedral subdivision obtained by intersecting $\mathcal{S}\left(v_{\infty}\right)$ with $\Delta^{\infty}$. That is, the cells of $\mathcal{S}_{\infty}$ are $\left\{Q_{\infty}:=\Delta_{Q} \cap \Delta^{\infty} \mid Q \subseteq P_{\infty}\right\}$. By Remark 6.5 and Remark 6.6, we have the following formulas for the equivariant limit mixed Hodge numbers of $H_{c}^{n-1}\left(X_{\text {gen }}\right)$ :

$$
\begin{aligned}
& u v \sum_{p, q} \sum_{\substack{\alpha \in \mathbb{Q} / \mathbb{Z} \\
\alpha \neq 1}} h^{p, q}\left(H_{c}^{n-1}\left(X_{\infty}\right)\right)_{\alpha} \alpha u^{p} v^{q} \\
& =\sum_{Q \subseteq P_{\infty}} v^{\operatorname{dim} \Delta_{Q}+1} l^{*}\left(\Delta_{Q},\left.v_{\infty}\right|_{\Delta_{Q}} ; u v^{-1}\right) l_{\Delta^{\infty}}\left(\mathcal{S}_{\infty}, Q_{\infty} ; u v\right), \\
& u v \sum_{p, q} h^{p, q}\left(H_{c}^{n-1}\left(X_{\infty}\right)\right)_{1} u^{p} v^{q}=\sum_{Q \subseteq P_{\infty}} v^{\operatorname{dim} Q+1} l^{*}\left(Q ; u v^{-1}\right) l_{\Delta^{\infty}}\left(\mathcal{S}_{\infty}, Q_{\infty} ; u v\right) .
\end{aligned}
$$

An algorithm to compute the equivariant limit mixed Hodge numbers above is given in [39, Section 5].

Remark 6.9 Recall from Remark 4.9 that $l_{\Delta^{\infty}}\left(\mathcal{S}_{\infty}, Q_{\infty} ; t\right)=t^{n-1-\operatorname{dim} Q} l_{\Delta^{\infty}}\left(\mathcal{S}_{\infty}, Q_{\infty} ; t^{-1}\right)$ has nonnegative, symmetric, unimodal coefficients. Also, by Remark 4.14, the coefficients of the local weighted $h^{*}$-polynomial are nonnegative, and we have symmetries $l^{*}(Q ; u)=$ $u^{\operatorname{dim} Q+1} l^{*}\left(Q ; u^{-1}\right)$ and $l^{*}\left(\Delta_{Q},\left.v_{\infty}\right|_{\Delta_{Q}} ; u\right)=u^{\operatorname{dim} \Delta_{Q}+1} \overline{l^{*}\left(\Delta_{Q},\left.v_{\infty}\right|_{\Delta_{Q}} ; u^{-1}\right)}$.

Recall also from (12) that we have the following equivalent formula for the equivariant refined limit Hodge-Deligne polynomial:

$$
u v w^{2} E\left(X_{\infty}, \widehat{\mu} ; u, v, w\right)=\left(u v w^{2}\right)^{n}+(-1)^{n-1} \sum_{S \subseteq\{1, \ldots, n\}}(-1)^{n-|S|} h^{*}\left(P^{S},\left.v_{\infty}\right|_{P} ; u, v, w\right) .
$$

After specializing at $v=w=1$, one obtains a formula for the spectrum at infinity of $f$ [47] that is equivalent to [39, Theorem 5.11]. Recall from (13) that specialization at $u=v=w=1$ yields a formula for the eigenvalues (with multiplicity) of the action of monodromy: 


$$
\sum_{\alpha \in \mathbb{Q} / \mathbb{Z}} \operatorname{dim} H_{c}^{n-1}\left(X_{\infty}\right)_{\alpha} \alpha=\sum_{\substack{Q \subseteq P_{\infty} \\ \operatorname{dim} \sigma\left(\Delta_{Q}\right)=\operatorname{dim} \Delta_{Q}}}(-1)^{n-1-\operatorname{dim} Q} \operatorname{Vol}(Q) \sum_{i=0}^{m(Q)-1}[i / m(Q)]
$$

where $\operatorname{Vol}(Q)$ is the normalized volume of $Q$ and $m(Q)$ is the lattice distance of $Q$ from the origin, i.e., $m(Q)$ is the minimal positive integer such that $\left.m(Q) v_{\infty}\right|_{\Delta_{Q}}$ is an affine function with respect to the lattice given by intersecting $M$ with the affine span of $\Delta_{Q}$. When $Q$ is empty, $\operatorname{Vol}(Q)=m(Q)=1$ and $\operatorname{dim} Q=-1$. The above is equivalent to a formula of Libgober and Sperber [35] for the zeta function at infinity of $f$.

Example 6.10 Following on with Example 3.1, Example 3.4 and Example 5.2, let $f=$ $\sum_{i=1}^{n} x_{i}^{m_{i}} \in \mathbb{C}\left[x_{1}, \ldots, x_{n}\right]$, for some $m_{i} \in \mathbb{Z}_{>0}$. Let $f_{1}, \ldots, f_{n}$ denote the standard basis vectors of $M_{\mathbb{R}}=\mathbb{R}^{n}$. Then $P$ is the convex hull of the origin and $\left\{m_{i} f_{i} \mid 1 \leq i \leq n\right\}$. In this case, $f: \mathbb{C}^{n} \rightarrow \mathbb{C}$ is a locally trivial fibration away from the origin, and it follows that the actions of monodromy at 0 and monodromy at infinity on cohomology are inverse to each other. In both cases, $v$ is $\mathbb{Q}$-affine and $\mathcal{S}(v)$ is trivial. For $\left(i_{1}, \ldots i_{n}\right) \in\left[1, m_{1}-1\right] \times$ $\cdots \times\left[1, m_{n}-1\right]$, let

$$
v_{\infty}\left(i_{1}, \ldots, i_{n}\right):=i_{1} / m_{1}+\cdots+i_{n} / m_{n}
$$

The action of monodromy is semi-simple, and, using Example 4.23, we compute the equivariant refined limit Hodge-Deligne polynomial $u v w^{2} E\left(X_{\infty}, \widehat{\mu} ; u, v, w\right)$ for monodromy at infinity:

$$
\sum_{\left(i_{1}, \ldots i_{n}\right) \in\left[1, m_{1}-1\right] \times \cdots \times\left[1, m_{n}-1\right]}^{\left(u v w^{2}\right)^{n}+(-1)^{n-1}}\left[v_{\infty}\left(i_{1}, \ldots, i_{n}\right)\right](u w)^{\left\lceil v_{\infty}\left(i_{1}, \ldots, i_{n}\right)\right\rceil}(v w)^{\left\lceil n-v_{\infty}\left(i_{1}, \ldots, i_{n}\right)\right\rceil} .
$$

For the corresponding invariant for monodromy at 0 , one simply reverses the roles of $u$ and $v$.

Example 6.11 Let $f=a_{0} x^{4}+a_{1} x^{5}+a_{2} x^{4} y^{2}+a_{3} x y^{5}+a_{4} y^{5}+a_{5} x y^{2}+a_{6} x^{2} y \in \mathbb{C}[x, y]$ for a general choice of $a_{0}, \ldots, a_{6} \in \mathbb{C}^{*}$. Then the pairs $(P, v)$ with their corresponding subdivisions $\mathcal{S}(v)$ in Example 6.7 and Example 6.8 are shown below. We have labeled all interior lattice points with nonzero weight. We may calculate the invariants above using Example 6.4. In particular, using the notation of Example 6.4, we have $\operatorname{dim} G r_{0}^{W} H_{c}^{1}\left(X_{\text {gen }}\right)=b=4$. Also, for monodromy at 0 , we have

$$
h_{0,0,1}^{*}\left(P, v_{0}\right)=2, h_{0,1,1}^{*}\left(P, v_{0}\right)=7+[1 / 3] .
$$

In this case, the action of monodromy on $G r_{1}^{W} H_{c}^{1}\left(X_{\text {gen }}\right)$ has 16 Jordan blocks of size 1,14 with eigenvalue 1 and 1 with eigenvalue $\exp (2 \pi \sqrt{-1} / 3)$ and $\exp (4 \pi \sqrt{-1} / 3)$, respectively, as well as 2 Jordan blocks of size 2 with eigenvalue 1 . For monodromy at infinity, we have

$$
\begin{aligned}
& h_{0,0,1}^{*}\left(P, v_{\infty}\right)=[1 / 2] \\
& h_{0,1,1}^{*}\left(P, v_{\infty}\right)=[7 / 10]+[9 / 10]+[1 / 3]+[1 / 2]+2[2 / 3]+3[5 / 6]
\end{aligned}
$$

In this case, the action of monodromy on $\mathrm{Gr}_{1}^{W} H_{c}^{1}\left(X_{\text {gen }}\right)$ has 18 Jordan blocks of size 1,3 with eigenvalues $\exp (2 \pi \sqrt{-1} / 6), \exp (2 \pi \sqrt{-1} / 3), \exp (4 \pi \sqrt{-1} / 3)$ and $\exp (10 \pi \sqrt{-1} / 6)$, respectively, 2 with eigenvalue -1 and 1 with eigenvalues $\exp (\pi \sqrt{-1} / 5), \exp (3 \pi \sqrt{-1} / 5)$, 
$\exp (7 \pi \sqrt{-1} / 5)$ and $\exp (9 \pi \sqrt{-1} / 5)$, respectively, as well as a single Jordan block of size 2 with eigenvalue -1 .

Monodromy at 0 Monodromy at $\infty$
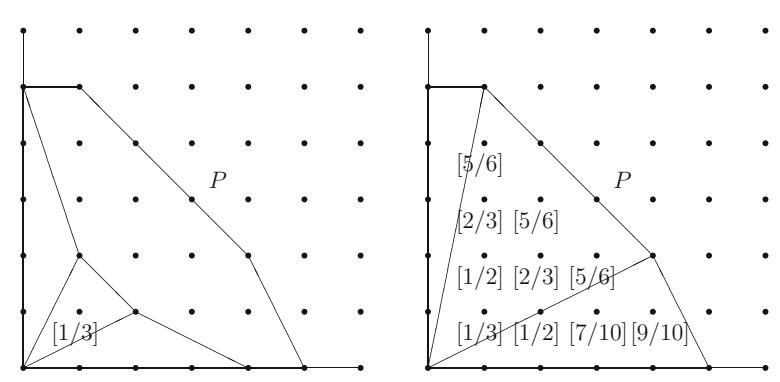

6.2 Jordan block structure of monodromy at infinity

We continue with the notation of Example 6.8 above, and let $f \in \mathbb{C}\left[x_{1}, \ldots, x_{n}\right]$ be convenient and schön at infinity, and let $X=\{f t=\lambda\} \subseteq \mathbb{K}^{n}$ for a general choice of $\lambda \in \mathbb{C}^{*}$. Recall that for every face $Q$ of the Newton polytope $\mathrm{NP}(f)$, we let $\Delta_{Q}$ denote the convex hull of $Q$ and the origin. Recall that $P=\Delta_{\mathrm{NP}(f)}$, and $P_{\infty}$ denotes the union of the faces at infinity, i.e., the faces of $P$ not containing the origin. Recall that $v_{\infty}$ is the piecewise $\mathbb{Q}$ affine function on $P$ with value 0 at the origin and value identically 1 on the faces at infinity of $P$, and the cells of $\mathcal{S}\left(v_{\infty}\right)$ are given by the union of $\left\{Q \mid Q \subseteq P_{\infty}\right\}$ and $\left\{\Delta_{Q} \mid Q \subseteq P_{\infty}\right\}$. Recall that $\Delta^{\infty}$ is the simplex $P \cap\left\{x_{1}+\cdots+x_{n}=\epsilon\right\}$ for fixed $\epsilon>0$ sufficiently small, and $\mathcal{S}_{\infty}$ denotes the regular, rational polyhedral subdivision of $\Delta^{\infty}$ obtained by intersecting $\mathcal{S}\left(v_{\infty}\right)$ with $\Delta^{\infty}$.

Following [39], one may use results of Broughton and Sabbah to read off the Jordan normal form of the action of monodromy on $H_{c}^{n-1}\left(X_{\text {gen }}\right)$ from the equivariant limit Hodge-Deligne polynomial. We outline the argument below. By Poincaré duality, we may work with usual cohomology $H^{n-1}\left(X_{\text {gen }}\right)$ rather than cohomology with compact supports. We write $H^{n-1}\left(X_{\text {gen }}\right)=H^{n-1}\left(X_{\text {gen }}\right)_{=1} \oplus H^{n-1}\left(X_{\text {gen }}\right)_{\neq 1}$, where $H^{n-1}\left(X_{\text {gen }}\right)_{=1}$ denotes the 1-eigenspace of $H^{n-1}\left(X_{\text {gen }}\right)$, and $H^{n-1}\left(X_{\text {gen }}\right)_{\neq 1}$ denotes the sum of the $\alpha$ eigenspaces, for $\alpha \neq 1$. The induced monodromy weight filtration $M_{\bullet}$ on $H^{n-1}\left(X_{\text {gen }}\right)$ may be written as $M_{\bullet}=\left(M_{=1}\right)_{\bullet} \oplus\left(M_{\neq 1}\right)_{\bullet}$, where $\left(M_{=1}\right)_{\bullet}$ and $\left(M_{\neq 1}\right)_{\bullet}$ are the induced filtrations on $H^{n-1}\left(X_{\text {gen }}\right)_{=1}$ and $H^{n-1}\left(X_{\text {gen }}\right)_{\neq 1}$, respectively. Let $N=\log T_{u}$ denote the nilpotent operator where $T_{u}$ is the unipotent part of monodromy acting on $H^{n-1}\left(X_{\text {gen }}\right)$, and consider the induced action of $N$ on $H^{n-1}\left(X_{\text {gen }}\right)_{=1}$ and $H^{n-1}\left(X_{\text {gen }}\right)_{\neq 1}$. As explained in detail in [39], using results of Broughton [9], we have the following special case of a deep result of Sabbah. Recall the definition of the weight filtration of a nilpotent operator from Definition 2.6.

Theorem 6.12 [48, Theorem 13.1] [39, Proposition A.1] Let $\in \mathbb{C}\left[x_{1}, \ldots, x_{n}\right]$ be convenient and schön at infinity, and let $X=\{f t=\lambda\} \subseteq \mathbb{K}^{n}$. Then $\left(M_{=1}\right)$. (respectively, $\left.\left(M_{\neq 1}\right)_{\bullet}\right)$ is equal to the $N$-weight filtration centered at $n$ (respectively, $n-1)$.

Using Poincaré duality together with the symmetries of Remark 6.9, the corollary below follows immediately from Example 6.8.

Corollary 6.13 Let $f \in \mathbb{C}\left[x_{1}, \ldots, x_{n}\right]$ be convenient and schön at infinity, and let $X=$ $\{f t=\lambda\} \subseteq \mathbb{K}^{n}$. Then we have following formulas for the equivariant limit mixed Hodge 
numbers of $H^{n-1}\left(X_{\text {gen }}\right)$ :

$$
\begin{aligned}
& u v \sum_{p, q} \sum_{\substack{\alpha \in \mathbb{Q} / \mathbb{Z} \\
\alpha \neq 1}} h^{p, q}\left(H^{n-1}\left(X_{\infty}\right)\right)_{\alpha} \alpha u^{p} v^{q} \\
& \quad=\sum_{Q \subseteq P_{\infty}} v^{\operatorname{dim} \Delta_{Q}+1} l^{*}\left(\Delta_{Q},\left.v_{\infty}\right|_{\Delta_{Q}} ; u v^{-1}\right) l_{\Delta^{\infty}}\left(\mathcal{S}_{\infty}, Q_{\infty} ; u v\right), \\
& \sum_{p, q} h^{p, q}\left(H^{n-1}\left(X_{\infty}\right)\right)_{1} u^{p} v^{q}=\sum_{Q \subseteq P_{\infty}} v^{\operatorname{dim} Q+1} l^{*}\left(Q ; u v^{-1}\right) l_{\Delta^{\infty}}\left(\mathcal{S}_{\infty}, Q_{\infty} ; u v\right),
\end{aligned}
$$

where the sum runs over all (possibly empty) faces at infinity.

Specializing the formulas in Corollary 6.13 by setting $u=v$ yields:

$$
\begin{aligned}
& u^{2} \sum_{p, q} \sum_{\substack{\alpha \in \mathbb{Q} / \mathbb{Z} \\
\alpha \neq 1}} h^{p, q}\left(H^{n-1}\left(X_{\infty}\right)\right)_{\alpha} \alpha u^{p+q} \\
& =\sum_{Q \subseteq P_{\infty}} u^{\operatorname{dim} \Delta_{Q}+1} l^{*}\left(\Delta_{Q},\left.v_{\infty}\right|_{\Delta_{Q}} ; 1\right) l_{\Delta^{\infty}}\left(\mathcal{S}_{\infty}, Q_{\infty} ; u^{2}\right), \\
& \sum_{p, q} h^{p, q}\left(H^{n-1}\left(X_{\infty}\right)\right)_{1} u^{p+q}=\sum_{Q \subseteq P_{\infty}} u^{\operatorname{dim} Q+1} l^{*}(Q ; 1) l_{\Delta^{\infty}}\left(\mathcal{S}_{\infty}, Q_{\infty} ; u^{2}\right) .
\end{aligned}
$$

The following result now follows directly from Theorem 6.12. For every face at infinity $Q$ of $P$, it follows from Remark 4.9 that we may write

$$
l_{\Delta \infty}\left(\mathcal{S}_{\infty}, Q_{\infty} ; t\right)=\sum_{i=0}^{\left\lfloor\frac{n-1-\operatorname{dim} Q}{2}\right\rfloor} \tilde{l}_{Q, i} t^{i}\left(1+t+\cdots+t^{n-1-\operatorname{dim} Q-2 i}\right)
$$

for some nonnegative integers $\left\{\tilde{l}_{Q, i} \mid 0 \leq i \leq\left\lfloor\frac{n-1-\operatorname{dim} Q}{2}\right\rfloor\right\}$. We define

$$
\tilde{l}_{\Delta \infty}\left(\mathcal{S}_{\infty}, Q_{\infty} ; t\right):=\sum_{i=0}^{\left\lfloor\frac{n-1-\operatorname{dim} Q}{2}\right\rfloor} \tilde{l}_{Q, i} t^{i}
$$

Corollary 6.14 Let $f \in \mathbb{C}\left[x_{1}, \ldots, x_{n}\right]$ be convenient and schön at infinity, and let $J_{k, \alpha}^{\infty}$ be the number of Jordan blocks of size $k$ with eigenvalue $\alpha$ for the action of monodromy at infinity on $H^{n-1}\left(f^{-1}(t)\right)$ for $t$ fixed and sufficiently large. Then

$$
\begin{aligned}
& \sum_{\substack{\alpha \in \mathbb{Q} / \mathbb{Z} \\
\alpha \neq 1}} \sum_{k} J_{n-k, \alpha}^{\infty} \alpha u^{k+2}=\sum_{Q \subseteq P_{\infty}} u^{\operatorname{dim} \Delta_{Q}+1} l^{*}\left(\Delta_{Q}, v_{\infty} \mid \Delta_{Q} ; 1\right) \tilde{l}_{\Delta}\left(\mathcal{S}_{\infty}, Q_{\infty} ; u^{2}\right), \\
& \sum_{k} J_{n-1-k, 1}^{\infty} u^{k+2}=\sum_{Q \subseteq P_{\infty}} u^{\operatorname{dim} Q+1} l^{*}(Q ; 1) \tilde{l}_{\Delta^{\infty}}\left(\mathcal{S}_{\infty}, Q_{\infty} ; u^{2}\right),
\end{aligned}
$$

where the sum runs over all (possibly empty) faces at infinity.

Example 6.15 Let $\partial \mathbb{R}_{\geq 0}^{n}$ denote the intersection of $\mathbb{R}_{\geq 0}^{n}$ with the union of the coordinate hyperplanes. Using either Example 4.10 and Example 4.15, or Example 4.24 and Example 6.2, one computes the number of Jordan blocks with the largest and second largest possible size: 


$$
\begin{gathered}
\sum_{\substack{\alpha \in \mathbb{Q} / \mathbb{Z} \\
\alpha \neq 1}} J_{n, \alpha}^{\infty} \alpha=h_{0,0, n-1}^{*}\left(P, v_{\infty}\right)=\sum_{\substack{Q \subseteq P_{\infty}, Q \nsubseteq \partial \mathbb{R}_{\geq 0}^{n} \\
\operatorname{dim} Q \in \operatorname{Qn}\left(\operatorname{In}\left(\Delta_{Q}\right) \cap \mathbb{Z}^{n}\right.}} w(v), \\
\sum_{\substack{\alpha \neq 1 \\
\alpha \neq 1}} J_{n-1, \alpha}^{\infty} \alpha=h_{0,1, n-1}^{*}\left(P, v_{\infty}\right)+h_{1,0, n-1}^{*}\left(P, v_{\infty}\right) \\
=\sum_{\substack{Q \subseteq P_{\infty}, Q \nsubseteq \partial \mathbb{R}_{\geq 0}^{n} \\
\operatorname{dim} Q=1}} \sum_{J_{n-1,1}^{\infty} \operatorname{Int}\left(\Delta_{Q}\right) \cap \mathbb{Z}^{n}} w(v)+\overline{w(v),} \\
\sum_{\substack{Q \subseteq P_{\infty}, Q \nsubseteq \partial \mathbb{R}_{\geq 0}^{n} \\
\operatorname{dim} Q \leq 1}} \#\left(\operatorname{Int}(Q) \cap \mathbb{Z}^{n}\right), \\
J_{n-2,1}^{\infty}=2 \cdot \sum_{\substack{Q \subseteq P_{\infty}, Q \nsubseteq \partial \mathbb{R}_{\geq 0}^{n} \\
\operatorname{dim} Q=2}} \#\left(\operatorname{Int}(Q) \cap \mathbb{Z}^{n}\right) .
\end{gathered}
$$

This reproves the main results of [39]. Namely, the first two equations are equivalent to [39, Theorem 1.1], and the second two equations are equivalent to [39, Theorem 1.2].

Example 6.16 As in Example 6.4, consider the case when $n=2$. Then $J_{1,1}^{\infty}=\#\left(\partial P \cap \mathbb{Z}_{>0}^{2}\right)$, and

$$
\begin{aligned}
& \sum_{\substack{\alpha \in \mathbb{Q} / \mathbb{Z} \\
\alpha \neq 1}} J_{2, \alpha}^{\infty} \alpha= \sum_{\substack{Q \subseteq P_{\infty}, Q \nsubseteq \partial \mathbb{R}_{\geq 0}^{2} \\
\operatorname{dim} Q=0}} \sum_{\substack{\alpha \in \mathbb{I n t}\left(\Delta_{Q}\right) \cap \mathbb{Z}^{2} \\
\alpha \neq 1}} w(v), \\
& \sum_{\substack{\alpha / \mathbb{Z} \\
\alpha, \alpha}} J_{1, \alpha}^{\infty} \alpha=\sum_{\substack{Q \subseteq P_{\infty} \\
\operatorname{dim} Q=1}} \sum_{v \in \operatorname{Int}\left(\Delta_{Q}\right) \cap \mathbb{Z}^{2}} w(v)+\overline{w(v) .}
\end{aligned}
$$

Example 6.17 Following on with Example 6.10, if $f=\sum_{i=1}^{n} x_{i}^{m_{i}} \in \mathbb{C}\left[x_{1}, \ldots, x_{n}\right]$, for some $m_{i} \in \mathbb{Z}_{>0}$, then the action of monodromy is semi-simple, and all Jordan blocks have size 1. With the notation of Example 6.10,

$$
\sum_{\alpha \in \mathbb{Q} / \mathbb{Z}} J_{1, \alpha}^{\infty} \alpha=\sum_{\left(i_{1}, \ldots i_{n}\right) \in\left[1, m_{1}-1\right] \times \cdots \times\left[1, m_{n}-1\right]}\left[v_{\infty}\left(i_{1}, \ldots, i_{n}\right)\right] \in \mathbb{Z}[\mathbb{Q} / \mathbb{Z}] .
$$

Example 6.18 As in Example 6.11, let $f=a_{0} x^{4}+a_{1} x^{5}+a_{2} x^{4} y^{2}+a_{3} x y^{5}+a_{4} y^{5}+$ $a_{5} x y^{2}+a_{6} x^{2} y \in \mathbb{C}[x, y]$ for a general choice of $a_{0}, \ldots, a_{6} \in \mathbb{C}^{*}$. The action of monodromy on $H^{1}\left(X_{\text {gen }}\right)$ has 22 Jordan blocks of size 1,4 with eigenvalue 1,3 with eigenvalues $\exp (2 \pi \sqrt{-1} / 6), \exp (2 \pi \sqrt{-1} / 3), \exp (4 \pi \sqrt{-1} / 3)$ and $\exp (10 \pi \sqrt{-1} / 6)$, respectively, 2 with eigenvalue -1 and 1 with eigenvalues $\exp (\pi \sqrt{-1} / 5), \exp (3 \pi \sqrt{-1} / 5)$, $\exp (7 \pi \sqrt{-1} / 5)$ and $\exp (9 \pi \sqrt{-1} / 5)$, respectively, as well as a single Jordan block of size 2 with eigenvalue -1 .

\subsection{Monodromy of Milnor fibers}

Finally, we present some local versions of the formulas above (see Example 6.7).

Consider a polynomial $f \in \mathbb{C}\left[x_{1}, \ldots, x_{n}\right]$ and consider the polynomial map $f: \mathbb{C}^{n} \rightarrow \mathbb{C}$. Assume that $f^{-1}(0) \subseteq \mathbb{C}^{n}$ has an isolated singularity at the origin. Restricting $f: \mathbb{C}^{n} \rightarrow \mathbb{C}$ to a small ball about the origin in $\mathbb{C}^{n}$, and replacing $\mathbb{C}$ with a sufficiently small punctured disk about the origin, gives a locally trivial fibration called the Milnor fibration. Fix a fiber $F_{0}$, called the Milnor fiber. A fundamental result of Milnor asserts that $F_{0}$ has the 
homotopy type of a wedge of $(n-1)$-spheres [38]. In particular, $H^{m}\left(F_{0}\right)=0$ unless $m=0, n-1$. Here the monodromy action on $H^{0}\left(F_{0}\right)=\mathbb{C}$ is trivial. In [55], Steenbrink introduced a mixed Hodge structure $\left(F^{\bullet}, M_{\bullet}\right)$ on $H^{n-1}\left(F_{0}\right)$. The weight filtration has the following description in terms of the induced monodromy map $T=T_{s} T_{u}$ on $H^{n-1}\left(F_{0}\right)$ (cf. Theorem 6.12). We write $H^{n-1}\left(F_{0}\right)=H^{n-1}\left(F_{0}\right)_{=1} \oplus H^{n-1}\left(F_{0}\right)_{\neq 1}$, where $H^{n-1}\left(F_{0}\right)_{=1}$ denotes the 1-eigenspace of $H^{n-1}\left(F_{0}\right)$, and $H^{n-1}\left(F_{0}\right)_{\neq 1}$ denotes the sum of the $\alpha$-eigenspaces, for $\alpha \neq 1$. Then $M_{\bullet}=\left(M_{=1}\right)_{\bullet} \oplus\left(M_{\neq 1}\right)_{\bullet}$, where $\left(M_{=1}\right) \bullet$ and $\left(M_{\neq 1}\right)$. are the induced filtrations on $H^{n-1}\left(F_{0}\right)_{=1}$ and $H^{n-1}\left(F_{0}\right)_{\neq 1}$, respectively. Let $N=\log T_{u}$ denote the nilpotent operator acting on $H^{n-1}\left(F_{0}\right)$, and consider the induced action of $N$ on $H^{n-1}\left(F_{0}\right)_{=1}$ and $H^{n-1}\left(F_{0}\right)_{\neq 1}$. Then $\left(M_{=1}\right)_{\bullet}$ (respectively, $\left(M_{\neq 1}\right)_{\bullet}$ ) is equal to the $N$-weight filtration centered at $n$ (respectively, $n-1)$ (see Definition 2.6). Denef and Loeser introduced the motivic Milnor fiber [16], which specializes under the equivariant Hodge-Deligne map $E_{\widehat{\mu}}$ to the equivariant Hodge-Deligne polynomial $E\left(F_{0}, \widehat{\mu} ; u, v\right) \in \mathbb{Z}[\mathbb{Q} / \mathbb{Z}][u, v]$ associated with $F_{0}$ with the above mixed Hodge structure. Explicitly,

$$
E\left(F_{0}, \widehat{\mu} ; u, v\right):=\sum_{p, q} \sum_{\alpha \in \mathbb{Q} / \mathbb{Z}} \sum_{m}(-1)^{m} h^{p, q}\left(H^{m}\left(F_{0}\right)\right)_{\alpha} \alpha u^{p} v^{q} .
$$

Recall that $\Gamma_{+}(f)=\mathrm{NP}(f)+\mathbb{R}_{\geq 0}^{n}$ denotes the Newton polyhedron of $f$, and $\Gamma_{f}$ denotes the union of the bounded faces of $\Gamma_{+}(f)$. Recall that for every bounded face $Q$ of $\Gamma_{+}(f), \Delta_{Q}$ denotes the convex hull of $Q$ and the origin. We assume that $f$ is convenient, i.e., $\Gamma_{+}(f)$ has nonzero intersection with each ray through a coordinate vector. We assume that $f$ is schön at 0 , meaning that $\left\{\left.f\right|_{Q}=0\right\} \subseteq\left(\mathbb{C}^{*}\right)^{n}$ defines a smooth hypersurface whenever $Q$ is a bounded face of $\Gamma_{+}(f)$. Note that this is a weaker condition than the condition that $f$ is schön in Example 6.7. Let $P_{+}$be the union of $\left\{\Delta_{Q} \mid Q \subseteq \Gamma_{f}\right\}$, and let $v_{0}$ be the piecewise $\mathbb{Q}$-affine function on $P_{+}$with value 1 at the origin and value 0 on $\Gamma_{f}$. Then the lattice polyhedral decomposition $\mathcal{S}\left(v_{0}\right)$ of $P_{+}$has cells $\left\{\Delta_{Q} \mid Q \subseteq \Gamma_{f}\right\}$ and $\left\{Q \subseteq \Gamma_{f}\right\}$. Although we will not need this, we note that even though $P_{+}$is not convex, one can apply all the combinatorial constructions and results of Sect. 4. With the notation of Example 6.7, the following formula for the motivic Milnor fiber is given in [40, Theorem 4.3] (cf. (14)):

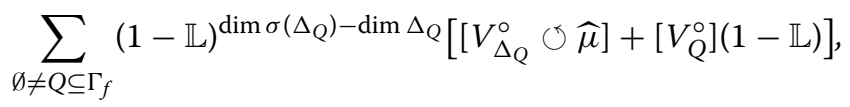

where $\sigma\left(\Delta_{Q}\right)$ is the smallest face of $P_{+}$containing $\Delta_{Q}$. Specializing the above expression via the equivariant Hodge-Deligne map and applying Theorem 5.11 yields the following formula for the equivariant Hodge-Deligne polynomial:

$$
\begin{aligned}
u v E\left(F_{0}, \widehat{\mu} ; u, v\right)= & \sum_{\emptyset \neq Q \subseteq \Gamma_{f}}(-1)^{\operatorname{dim} Q}(1-u v)^{\operatorname{dim} \sigma\left(\Delta_{Q}\right)-\operatorname{dim} \Delta_{Q}\left[h^{*}\left(\Delta_{Q},\left.v_{0}\right|_{\Delta_{Q}} ; u, v\right)\right.} \\
& \left.+(u v-1) h^{*}(Q ; u, v)\right],
\end{aligned}
$$

where $h^{*}(Q ; u, v)$ is the weighted limit mixed $h^{*}$-polynomial with respect to the convex graph that is identically zero. This gives the following expression for $\sum_{p, q} \sum_{\alpha \in \mathbb{Q} / \mathbb{Z}} h^{p, q}$ $\left(H^{n-1}\left(F_{0}\right)\right)_{\alpha} \alpha u^{p} v^{q}$ :

$$
\sum_{Q \subseteq \Gamma_{f}}(-1)^{n-1-\operatorname{dim} Q_{(1-u v}}\left(1-\operatorname{dim} \sigma\left(\Delta_{Q}\right)-\operatorname{dim} \Delta_{Q}\left[h^{*}\left(\Delta_{Q},\left.v_{0}\right|_{\Delta_{Q}} ; u, v\right)+(u v-1) h^{*}(Q ; u, v)\right] .\right.
$$

Remark 6.19 The formula below should be compared to (15). Note that $E\left(F_{0}, \widehat{\mu} ; 1,1\right)=$ $\sum_{\alpha \in \mathbb{Q} / \mathbb{Z}} \sum_{m}(-1)^{m} \operatorname{dim} H^{m}\left(F_{0}\right)_{\alpha} \alpha$ determines the eigenvalues (with multiplicity) of the 
action of monodromy. Specializing the above formula at $u=v=1$, and applying Example 4.12, gives the following formula:

$$
\sum_{\alpha \in \mathbb{Q} / \mathbb{Z}} \operatorname{dim} H^{n-1}\left(F_{0}\right)_{\alpha} \alpha=\sum_{\substack{Q \subseteq \Gamma_{f} \\ \operatorname{dim} \sigma\left(\Delta_{Q}\right)=\operatorname{dim} \Delta_{Q}}}(-1)^{n-1-\operatorname{dim} Q} \operatorname{Vol}(Q) \sum_{i=0}^{m(Q)-1}[i / m(Q)],
$$

where $\operatorname{Vol}(Q)$ is the normalized volume of $Q$ and $m(Q)$ is the lattice distance of $Q$ from the origin, i.e., $m(Q)$ is the minimal positive integer such that $\left.m(Q) v_{0}\right|_{\Delta_{Q}}$ is an affine function with respect to the lattice given by intersecting $M$ with the affine span of $\Delta_{Q}$. When $Q$ is empty, $\operatorname{Vol}(Q)=m(Q)=1$ and $\operatorname{dim} Q=-1$. This is equivalent to a famous formula of Varchenko [61] for the zeta function of monodromy of $F_{0}$. As in Remark 2.4, we obtain a formula for $\operatorname{dim} H^{n-1}\left(F_{0}\right)$ originally due to Kouchnirenko [34].

As in previous sections, expanding the definitions above, simplifying and using Remark 6.6, yields the following 'nonnegative' formulas for the equivariant mixed Hodge numbers of $F_{0}$. Let $\Delta^{0}$ be the simplex $P_{+} \cap\left\{x_{1}+\cdots+x_{n}=\epsilon\right\}$ for fixed $\epsilon>0$ sufficiently small. Let $\mathcal{S}_{0}$ denote the regular, rational polyhedral subdivision obtained by intersecting $\mathcal{S}\left(v_{0}\right)$ with $\Delta^{0}$. That is, the cells of $\mathcal{S}_{0}$ are $\left\{Q_{0}:=\Delta_{Q} \cap \Delta^{0} \mid Q \subseteq \Gamma_{f}\right\}$.

Theorem 6.20 Let $f \in \mathbb{C}\left[x_{1}, \ldots, x_{n}\right]$ be a complex polynomial such that $f^{-1}(0)$ admits an isolated singularity at the origin. Assume further that $f$ is convenient and schön at 0. With the notation above, we have the following formulas for the equivariant mixed Hodge numbers of the cohomology of the associated Milnor fiber $F_{0}$ :

$$
\begin{aligned}
& u v \sum_{p, q} \sum_{\substack{\alpha \in \mathbb{Q} / \mathbb{Z} \\
\alpha \neq 1}} h^{p, q}\left(H^{n-1}\left(F_{0}\right)\right)_{\alpha} \alpha u^{p} v^{q} \\
& =\sum_{Q \subseteq \Gamma_{f}} v^{\operatorname{dim} \Delta_{Q}+1} l^{*}\left(\Delta_{Q},\left.v_{0}\right|_{\Delta_{Q}} ; u v^{-1}\right) l_{\Delta^{0}}\left(\mathcal{S}_{0}, Q_{0} ; u v\right), \\
& \sum_{p, q} h^{p, q}\left(H^{n-1}\left(F_{0}\right)\right)_{1} u^{p} v^{q}=\sum_{Q \subseteq \Gamma_{f}} v^{\operatorname{dim} Q+1} l^{*}\left(Q ; u v^{-1}\right) l_{\Delta^{0}}\left(\mathcal{S}_{0}, Q_{0} ; u v\right),
\end{aligned}
$$

where the sum runs over all (possibly empty) bounded faces of the Newton polyhedron off.

We note that an algorithm to compute the equivariant mixed Hodge numbers of the cohomology of the associated Milnor fiber $F_{0}$, as well as formulas in special cases, was given by Matsui and Takeuchi in [40], extending work of Danilov [13] and Tanabé [58].

Remark 6.21 There is a striking symmetry between the formulas for monodromy at infinity and monodromy of the Milnor fiber in Corollary 6.13 and Theorem 6.20, respectively. The existence of such a symmetry was first observed by Matsui and Takeuchi in [40].

The following corollary is immediate from the description of the weight filtration on the cohomology of $F_{0}$ and Definition 2.6 and should be compared to Corollary 6.14. For every bounded face $Q$ of $\Gamma_{f}$, it follows from Remark 4.9 that we may write

$$
l_{\Delta^{0}}\left(\mathcal{S}_{0}, Q_{0} ; t\right)=\sum_{i=0}^{\left\lfloor\frac{n-1-\operatorname{dim} Q}{2}\right\rfloor} \tilde{l}_{Q, i} t^{i}\left(1+t+\cdots+t^{n-1-\operatorname{dim} Q-2 i}\right),
$$


for some nonnegative integers $\left\{\widetilde{l}_{Q, i} \mid 0 \leq i \leq\left\lfloor\frac{n-1-\operatorname{dim} Q}{2}\right\rfloor\right\}$. We define

$$
\tilde{l}_{\Delta^{0}}\left(\mathcal{S}_{0}, Q_{0} ; t\right):=\sum_{i=0}^{\left\lfloor\frac{n-1-\operatorname{dim} Q}{2}\right\rfloor} \tilde{l}_{Q, i} t^{i} .
$$

Corollary 6.22 Let $f \in \mathbb{C}\left[x_{1}, \ldots, x_{n}\right]$ be a complex polynomial such that $f^{-1}(0)$ admits an isolated singularity at the origin. Assume further that $f$ is convenient and schön at 0 . Let $J_{k, \alpha}^{0}$ be the number of Jordan blocks of size $k$ with eigenvalue $\alpha$ for the action of monodromy on the cohomology of the Milnor fiber $H^{n-1}\left(F_{0}\right)$. Then

$$
\begin{aligned}
& \sum_{\substack{\alpha \in \mathbb{Q} / \mathbb{Z} \\
\alpha \neq 1}} \sum_{k} J_{n-k, \alpha}^{0} \alpha u^{k+2}=\sum_{Q \subseteq \Gamma_{f}} u^{\operatorname{dim} \Delta_{Q}+1} l^{*}\left(\Delta_{Q},\left.v_{0}\right|_{\Delta_{Q}} ; 1\right) \tilde{l}_{\Delta^{0}}\left(\mathcal{S}_{0}, Q_{0} ; u^{2}\right), \\
& \sum_{k} J_{n-1-k, 1}^{0} u^{k+2}=\sum_{Q \subseteq \Gamma_{f}} u^{\operatorname{dim} Q+1} l^{*}(Q ; 1) \tilde{l}_{\Delta^{0}}\left(\mathcal{S}_{0}, Q_{0} ; u^{2}\right),
\end{aligned}
$$

where the sum runs over all (possibly empty) bounded faces of the Newton polyhedron off.

Example 6.23 We have the following analogue of Example 6.15. Let $\partial \mathbb{R}_{\geq 0}^{n}$ denote the intersection of $\mathbb{R}_{\geq 0}^{n}$ with the union of the coordinate hyperplanes. Then we have the following formulas for the number of Jordan blocks with the largest and second largest possible size:

$$
\begin{aligned}
& \sum_{\substack{\alpha \in \mathbb{Q} / \mathbb{Z} \\
\alpha \neq 1}} J_{n, \alpha}^{0} \alpha=\sum_{\substack{Q \subseteq \Gamma_{f}, Q \nsubseteq \partial \mathbb{R}_{\geq 0}^{n} \\
\operatorname{dim} Q=0}} \sum_{v \in \operatorname{Int}\left(\Delta_{Q}\right) \cap \mathbb{Z}^{n}} w(v), \\
& \sum_{\substack{\alpha \in \mathbb{Q} / \mathbb{Z} \\
\alpha \neq 1}} J_{n-1, \alpha}^{0} \alpha=\sum_{\substack{Q \subseteq \Gamma_{f}, Q \nsubseteq \partial \mathbb{R}_{\geq 0}^{n} \\
\operatorname{dim} Q=1}} \sum_{v \in \operatorname{Int}\left(\Delta_{Q}\right) \cap \mathbb{Z}^{n}} w(v)+\overline{w(v)} \\
& J_{n-1,1}^{0}=\sum_{\substack{Q \subseteq \Gamma_{f}, Q \nsubseteq \partial \mathbb{R}_{\geq 0}^{n} \\
\operatorname{dim} Q \leq 1}} \#\left(\operatorname{Int}(Q) \cap \mathbb{Z}^{n}\right), \\
& J_{n-2,1}^{0}=2 \cdot \sum_{\substack{Q \subseteq \Gamma_{f}, Q \nsubseteq \partial \mathbb{R}_{\geq 0}^{n} \\
\operatorname{dim} Q=2}} \#\left(\operatorname{Int}(Q) \cap \mathbb{Z}^{n}\right) .
\end{aligned}
$$

This reproves the main results of [40]. Namely, the first two equations are equivalent to [40, Theorem 1.1], and the second two equations are equivalent to [40, Theorem 1.2]. Also, the third equation is originally due to van Doorn and Steenbrink [60].

Example 6.24 We have the following analogue of Example 6.16. Consider the case when $n=2$. Then $J_{1,1}^{0}=\#\left(\Gamma_{f} \cap \mathbb{Z}_{>0}^{2}\right)$, and

$$
\begin{aligned}
& \sum_{\substack{\alpha \in \mathbb{Q} / \mathbb{Z} \\
\alpha \neq 1}} J_{2, \alpha}^{0} \alpha=\sum_{\substack{Q \subseteq \Gamma_{f}, Q \nsubseteq \partial \mathbb{R}_{\geq 0}^{2} \\
\operatorname{dim} Q=0}} \sum_{\substack{ \\
v \in \operatorname{Int}\left(\Delta_{Q}\right) \cap \mathbb{Z}^{2}}} w(v), \\
& \sum_{\substack{\alpha \in \mathbb{Q} / \mathbb{Z} \\
\alpha \neq 1}} J_{1, \alpha}^{0} \alpha=\sum_{\substack{Q \subseteq \Gamma_{f} \\
\operatorname{dim} Q=1}} \sum_{v \in \operatorname{Int}\left(\Delta_{Q}\right) \cap \mathbb{Z}^{2}} w(v)+\overline{w(v)} .
\end{aligned}
$$

Example 6.25 We have the following analogue of Example 6.17. Following on with Example 6.10, if $f=\sum_{i=1}^{n} x_{i}^{m_{i}} \in \mathbb{C}\left[x_{1}, \ldots, x_{n}\right]$, for some $m_{i} \in \mathbb{Z}_{>0}$, then the action of mon- 
odromy is semi-simple, and all Jordan blocks have size 1. With the notation of Example 6.10,

$$
\sum_{\alpha \in \mathbb{Q} / \mathbb{Z}} J_{1, \alpha}^{0} \alpha=\sum_{\left(i_{1}, \ldots i_{n}\right) \in\left[1, m_{1}-1\right] \times \cdots \times\left[1, m_{n}-1\right]}\left[v_{\infty}\left(i_{1}, \ldots, i_{n}\right)\right] \in \mathbb{Z}[\mathbb{Q} / \mathbb{Z}] .
$$

Example 6.26 The following should be compared to Example 6.18. As in Example 6.11, let $f=a_{0} x^{4}+a_{1} x^{5}+a_{2} x^{4} y^{2}+a_{3} x y^{5}+a_{4} y^{5}+a_{5} x y^{2}+a_{6} x^{2} y \in \mathbb{C}[x, y]$ for a general choice of $a_{0}, \ldots, a_{6} \in \mathbb{C}^{*}$. The action of monodromy on $H^{1}\left(F_{0}\right)$ is semi-simple and has 4 Jordan blocks of size 1,2 with eigenvalue 1 and 1 with eigenvalues $\exp (2 \pi \sqrt{-1} / 3)$ and $\exp (4 \pi \sqrt{-1} / 3)$, respectively.

\section{Acknowledgements}

The author would like to thank Anthony Henderson for some useful comments.

Received: 8 May 2016 Accepted: 30 January 2017

Published online: 10 April 2017

\section{References}

1. Athanasiadis, C.: Flag subdivisions and $\gamma$-vectors. Pac. J. Math. 259, 257-278 (2012)

2. Athanasiadis, C., Savvidou, C.: A symmetric unimodal decomposition of the derangement polynomial of type $B$. Preprint. arXiv:1303.2302

3. Batyrev, V., Borisov, L:: Mirror duality and string-theoretic Hodge numbers. Invent. Math. 126(1), 183-203 (1996)

4. Borisov, L., Chen, L., Smith, G.: The orbifold Chow ring of toric Deligne-Mumford stacks. J. Am. Math. Soc. 18(1), 193-215 (2005)

5. Borisov, L., Mavlyutov, A.: String cohomology of Calabi-Yau hypersurfaces via mirror symmetry. Adv. Math. 180(1), 355-390 (2003)

6. Batyrev, V., Nill, B.: Combinatorial aspects of mirror symmetry, integer points in polyhedra. Contemp. Math. 452, 35-66 (2008)

7. Bittner, F.: On motivic zeta functions and the motivic nearby fiber. Math. Z. 249(1), 63-83 (2005)

8. Bittner, F.: The universal Euler characteristic for varieties of characteristic zero. Comp. Math. 140(1), 1011-1032 (2004)

9. Allen, S.: Broughton, Milnor numbers and the topology of polynomial hypersurfaces. Invent. Math. 92, 217-241 (1988)

10. Cappell, S., Maxim, L., Shaneson, J.: Equivariant genera of complex algebraic varieties. Int. Math. Res. Not. 11, 2013-2037 (2009)

11. Chen, W., Ruan, Y.: Orbifold Gromov-Witten theory, orbifolds in mathematics and physics. Contemp. Math. 310 25-85 (2002)

12. Chen, W., Ruan, Y.: A new cohomology theory of orbifold. Commun. Math. Phys. 248(1), 1-31 (2004)

13. Danilov, V.: Newton polyhedra and vanishing cohomology. Funct. Anal. Appl. 13, 103-115 (1979)

14. Danilov, V., Khovanskiĭ, A.: Newton polyhedra and an algorithm for calculating Hodge-Deligne numbers. Izv. Akad. Nauk SSSR Ser. Mat. 50(5), 925-945 (1986)

15. Deligne, P.: Théorie de Hodge. I, Actes du Congrès International des Mathématiciens (Nice, 1970). Gauthier-Villars, Paris (1971)

16. Denef, J., Loeser, F.: Motivic Igusa zeta functions. J. Algebraic Geom. 7(3), 505-537 (1998)

17. Denef, J., Loeser, F.: Geometry on arc spaces of algebraic varieties. European Congress of Mathematics, Vol. I (Barcelona, 2001), vol. 201, pp. 327-348. Progr. Math. Birkhäuser, Basel (2001)

18. Dimca, A., Némethi, A.: On the monodromy of complex polynomials. Duke Math. J. 108, 199-209 (2001)

19. Dimca, A., Lehrer, G.: Purity and equivariant weight polynomials, algebraic groups and Lie groups. Austral. Math. Soc. Lect. Ser. 9, 161-181 (1997)

20. Ehrhart, E.: Sur un probléme de géométrie diophantienne linéaire. I. Polyédres et réseaux. J. Reine Angew. Math. 226 129 (1967)

21. Ehrhart, E:: Sur un probléme de géométrie diophantienne linéaire. II. Systémes diophantiens linéaires. Angew. Math. 227, 2549 (1967)

22. Ehrhart, E.: Démonstration de la loi de réciprocité du polyédre rationnel. C. R. Acad. Sci. Paris Sér. A 265, A91-A94 (1967)

23. Esterov, A., Takeuchi, K.: Motivic Milnor fibers over complete intersection varieties and their virtual Betti numbers. Int. Math. Res. Not. 15, 3567-3613 (2012)

24. Fulton, W.: Introduction to toric varieties, annals of mathematics studies. In: Roever, W.H. (ed.) Lectures in 3 Geometry, vol. 131. Princeton University Press, Princeton (1993)

25. Helm, D., Katz, E.: Monodromy filtrations and the topology of tropical varieties. Can. J. Math. 64(4), 845-868 (2012)

26. Jensen, A.N.: Gfan, a software system for Gröbner fans and tropical varieties. http://home.imf.au.dk/jensen/software/ gfan/gfan.html 
27. Jensen, A.N., Markwig, H., Markwig, T.: tropical.lib. A SINGULAR 3.0 library for computations in tropical geometry. http://www.mathematik.uni-kl.de/ keilen/en/tropical.html

28. Katz, E., Stapledon, A.: Tropical geometry and the motivic nearby fiber. Compos. Math. 148(1), 269-294 (2012)

29. Katz, E., Stapledon, A.: Local $h$-polynomials, invariants of subdivisions, and mixed Ehrhart theory. Preprint. arXiv:1411.7736

30. Katz, E., Stapledon, A.: Tropical geometry, the motivic nearby fiber and limit mixed Hodge numbers of hypersurfaces. Preprint. arXiv:1404.3000

31. Gelfand, I.M., Kapranov, M.M., Zelevinsky, A.V.: Discriminants, Resultants, and Multidimensional Determinants. Birkhäuser, Boston (1994)

32. Gubler, W.: A guide to tropicalizations, algebraic and combinatorial aspects of tropical geometry. Contemp. Math. 589, 125-190 (2013)

33. Khovanskiï, A.: Newton polyhedra, and toroidal varieties. Functional. Anal. i Priložen. 11(4), 56-64 (1977)

34. Kouchnirenko, A.: Polyedres de Newton et nombres de Milnor. Invent. Math. 32, 1-31 (1976)

35. Libgober, A., Sperber, S.: On the zeta function of monodromy of a polynomial map. Compos. Math. 95, 287-307 (1995)

36. Loera Jand Santo, F.: Triangulations: Structures for Algorithms and Applications. Springer, Berlin (2010)

37. Luxton, M., Zhunhua, Q:: Some results on tropical compactifications. Trans. Am. Math. Soc. 363(9), 4853-4876 (2011)

38. Milnor, J.: Singular points of complex hypersurfaces. Ann. Math. Stud. 61, 130 (1968)

39. Matsui, Y., Takeuchi, K.: Monodromy at infinity of polynomial maps and Newton polyhedra (with an appendix by C. Sabbah). Int. Math. Res. Not. IMRN 8, 1691-1746 (2013)

40. Matsui, Y., Takeuchi, K.: Motivic Milnor fibers and Jordan normal forms of Milnor monodromies. Publ. Res. Inst. Math. Sci. (in press). arXiv: 1202.5076

41. Nill, B., Schepers, J.: Combinatorial questions related to stringy E-polynomials of Gorenstein polytopes. In: Altmann, K., et al. (eds.) Toric Geometry. Oberwolfach Report No. 21, pp. 62-64 (2012)

42. Oda, T., Park, H.S.: Linear Gale transforms and GelfandKapranovZelevinskij decompositions. Tohoku Math. J. $\mathbf{4 3}(3)$, $375399(1991)$

43. Peters, C.: Motivic aspects of Hodge theory. TIFR-Lect. Notes Math. 92 (2010)

44. Peters, C., Steenbrink, J.: Mixed Hodge Structures, vol. 52. Springer, Berlin (2008)

45. Raibaut, M.: Fibre de Milnor motivique 'a linfini. C. R. Math. Acad. Sci. Paris 348(7-8), 419-422 (2010)

46. Richter-Gebert, J., Sturmfels, B., Theobald, T.: First steps in tropical geometry, Idempotent mathematics and mathematical physics. Contemp. Math. 377, 289-317 (2005)

47. Sabbah, C.: Monodromy at infinity and Fourier transform. Publ. Res. Inst. Math. Sci. 33, 643-685 (1997)

48. Sabbah, C.: Hypergeometric periods for a tame polynomial. Port. Math. 63, 173-226 (2006)

49. Schulze, M.: Algorithms for the Gauss-Manin connection. J. Symb. Comput. 32(5), 549-564 (2001)

50. Stanley, R.: Decompositions of rational convex polytopes. Ann. Discrete Math. 6, 333342 (1980)

51. Stanley, R.: Subdivisions and local $h$-vectors. J. Am. Math. Soc. 5(4), 805-851 (1992)

52. Stapledon, A.: Weighted Ehrhart theory and orbifold cohomology. Adv. Math. 219(1), 63-88 (2008)

53. Stapledon, A.: Motivic integration on toric stacks. Commun. Algebra 37(11), 3943-3965 (2009)

54. Stapledon, A.: Representations on the cohomology of hypersurfaces and mirror symmetry. Adv. Math. 226(6), 5268$5297(2011)$

55. Steenbrink, J.: Mixed Hodge structures on the vanishing cohomology. In: Holm, P. (ed.) Real and Complex Singularities, pp. 523-563. Sijthoff \& Noordhoff, Alphen van den Rijn (1977)

56. Steenbrink, J.: Motivic Milnor fibre for nondegenerate function germs on toric singularities. Preprint. arXiv:1310.6914

57. Takeuchi, K., Tibar, M., Monodromies at infinity of non-tame polynomials. Preprint. arXiv:1208.4584

58. Tanabé, S.: Combinatorial aspects of the mixed Hodge structure. RIMS Kokyuroku 1374, 15-39 (2004)

59. Tevelev, J.: Compactifications of subvarieties of tori. Am. J. Math. 129(4), 1087-1104 (2007)

60. van Doorn, M.G.M., Steenbrink, J.: A supplement to the monodromy theorem. Abh. Math. Sem. Univ. Hamburg $\mathbf{5 9}$ 225-233 (1989)

61. Varchenko, A.: Zeta-function of monodromy and Newtons diagram. Invent. Math. 37, 253-262 (1976)

\section{Submit your manuscript to a SpringerOpen ${ }^{\odot}$ journal and benefit from:}

$\checkmark$ Convenient online submission

$\checkmark$ Rigorous peer review

- Immediate publication on acceptance

- Open access: articles freely available online

- High visibility within the field

- Retaining the copyright to your article

Submit your next manuscript at $\boldsymbol{s p r i n g e r o p e n . c o m ~}$ 